\title{
Interpolação de dados faltantes em séries de imagens de satélite
}

\author{
Vinicius Soares Martins Alves
}

\author{
DiSSERTAÇÃO APRESENTADA AO \\ INSTITUTO DE MATEMÁTICA E EsTATÍSTICA \\ dA Universidade de SÃo Paulo \\ PARA OBTENÇÃO DO TÍTULO DE \\ Mestre EM Cî̂NCIAS
}

Programa: Programa de Pós-graduação em Estatística

Orientadora: Prof ${ }^{a}$. Dra ${ }^{\mathrm{a}}$. Chang Chiann

Durante o desenvolvimento deste trabalho o autor recebeu auxílio financeiro do CNPq

São Paulo

30 de Agosto de 2021 



\title{
Interpolação de dados faltantes em séries de imagens de satélite
}

\author{
Vinicius Soares Martins Alves
}

Esta versão da dissertação contém as correções e alterações sugeridas pela Comissão Julgadora durante a defesa da versão original do trabalho, realizada em 30 de Agosto de 2021.

Uma cópia da versão original está disponível no Instituto de Matemática e Estatística da Universidade de São Paulo.

Comissão Julgadora:

- Prof ${ }^{a}$. Dr ${ }^{\mathrm{a}}$. Chang Chiann (orientadora) - IME-USP

- Prof. Dr. Marcelo Magalhães Taddeo - UFBA

- Prof ${ }^{a}$. Dra ${ }^{a}$ Thelma Sáfadi - UFLA 



\section{Resumo}

Vinicius Soares Martins Alves. Interpolação de dados faltantes em séries de imagens de satélite. Dissertação (Mestrado). Instituto de Matemática e Estatística, Universidade de São Paulo, São Paulo, 2021.

O objetivo deste trabalho é comparar modelos de predição para a interpolação de dados faltantes em conjuntos de dados espaço-temporais dispostos em grade, comumente oferecidos através de sistemas de sensoriamento remoto. $\mathrm{O}$ método de Krigagem Universal, usual para essa aplicação, é computacionalmente intenso e seu uso pode ser inviável num contexto em que o volume de dados é maior. Uma classe de modelos mistos chamada Krigagem de Posto Fixo (FRK) visa reduzir a complexidade computacional desse procedimento e é comparada com modelos espaço-temporais hierárquicos que se beneficiam da relação entre a função de covariância de Matérn e a solução de equações diferencias parciais estocásticas. Simulações apontam melhores resultados para os métodos de maior complexidade computacional, mas a redução no tempo de execução é substancial para os novos modelos, com desempenho satisfatório sobretudo para os modelos hierárquicos. Por fim, para ilustração, os novos modelos são aplicados para um conjunto de dados obtidos de um satélite equipado com sensores de temperatura de superfície para uma área da Região Metropolitana de São Paulo.

Palavras-chave: Sensoriamento remoto. Krigagem. Dados espaço-temporais. Estatística espacial. Séries temporais. Modelos Hierárquicos. 



\begin{abstract}
Vinicius Soares Martins Alves. Interpolation of Missing Data on Satellite Image

Series. Thesis (Masters). Institute of Mathematics and Statistics, University of São Paulo, São Paulo, 2021.
\end{abstract}

The objective of this work is to compare predictive models for the interpolation of missing values in spatio-temporal data sets arranged in a grid, commonly available through remote sensing systems. The Universal Kriging method, usual for this application, is computationally intensive and its usage may be unfeasible in a context where the volume of data is large. A class of mixed models called Fixed Rank Kriging aims to reduce the computational complexity of this procedure and these models are compared with hierarchical spatio-temporal models that benefit from the relationship between the Matern covariance function and the solution of stochastic partial differential equations. Simulations show better results for the methods with greater computational complexity, but the improvement in execution time is substantial for the new methods, with adequate results especially using the hierarchical models. Finally, for illustration, the new models are applied to a data set obtained from a satellite equipped with surface temperature sensors for an area in the Metropolitan Region of São Paulo.

Keywords: Remote sensing. Kriging. Spatio-temporal data. Spatial statistics. Time series. Hierarchical models. 



\section{Lista de Figuras}

2.1 Processo estocástico, com cada variável $Z(t)$ definida em $(\Omega, A, P) \ldots \ldots$

2.2 Duas imagens simuladas exemplificando o conceito de isotropia. . . . . . 9

2.3 Componentes de um semivariograma como função da distância $\|\boldsymbol{h}\|$. . 12

4.1 Exemplo de Triangulação, usada na simulação desse trabalho. Um vértice de triângulo para cada quatro vértices de área (Triangulação 2), com triângulos a mais nas bordas para as fronteiras. . . . . . . . . . .

5.1 Exemplo de simulação de um conjunto com área 32x32 pixels em 64 tempos e sazonalidade de 16 tempos. Nessa figura, a cor dos pontos representa o valor simulado. . . . . . . . . . . . . . . 33

5.2 A mesma simulação apresentada acima, com dados removidos. . . . . . . 33

5.3 Predições para a Simulação apresentada na Figura 5.1, com os métodos descritos nessa seção. . . . . . . . . . . . . . . . . . .

5.4 Gráficos de dispersão entre o valor real e o valor predito para as observações que estão no conjunto. . . . . . . . . . . . . . . 38

5.5 Gráficos de dispersão entre o valor real e o valor predito para as observações que foram removidas do conjunto simulado. . . . . . . . . . 38

5.6 Gráficos de dispersão entre o erro quadrático médio entre as observações mantidas no conjunto em cada dia pelo número de observações faltantes no conjunto. . . . . . . . . . . . . . . . .

5.7 Gráficos de dispersão entre o erro quadrático médio de predição entre as observações suprimidas do conjunto em cada dia pelo número de observações faltantes no conjunto. . . . . . . . . . . . . . .

5.8 Viés das predições para a Simulação apresentada na Figura 5.1, com os métodos descritos nessa seção, e marcado pelas cores indicadas na escala.

5.9 Erro padrão estimado das predições para a Simulação apresentada na Figura 5.1 , com os métodos descritos nessa seção. . . . . . . . . . . . . . 
5.10 Intervalo das predições para a validar a simulação apresentada na Figura 5.1, com os métodos descritos nessa seção. A cor roxa indica que o valor real para o ponto ficou dentro do intervalo de predição, e a cor branca indica que o valor real ficou fora do intervalo. . . . . . . . . . . . .

5.11 Boxplots com os dados apresentados nas Tabelas 5.1 e 5.2: EQM - predições para todas as observações do conjunto de dados. . . . . . . . . . . . . .

5.12 Boxplots com os dados apresentados nas Tabelas 5.3 e 5.4: EQM - predições associadas às observações mantidas no conjunto de dados. . . . . . . . . 46

5.13 Boxplots com os dados apresentados nas Tabelas 5.5 e 5.6: EQM - predições associadas às observações suprimidas do conjunto de dados. . . . . . . . 46

6.1 Localização da área utilizada na análise na Região Metropolitana de São Paulo (EMPLASA, 2019). . . . . . . . . . . . . . . . 51

6.2 Ortofoto com a área utilizada no estudo em destaque (EMPLASA, 2010). . 51

6.3 Imagens que representam a temperatura de superfície $(K)$ nas áreas da região selecionada. A imagem 1 representa o mês de julho de 2009 e cada imagem representa um mês. . . . . . . . . . . . . . . . . . 53

6.4 Quantidade de áreas com valores observados, por imagem. . . . . . . . . 53

6.5 Gráficos das séries temporais de temperatura de superfície para quatro áreas de $1 \mathrm{Km}^{2}$ dentre as 729 que fazem parte do conjunto de dados selecionado. . . . . . . . . . . . . . . . . . .

6.6 Predições para preencher o conjunto de dados apresentado na Figura 6.3, para cada um dos métodos avaliados. . . . . . . . . . . . . . .

6.7 Erros padrões estimados para a predição de cada localidade do conjunto de dados. Nota-se a diferença nas escalas. . . . . . . . . . . . . . . . .

6.8 Gráficos de dispersão entre o valor real e o valor predito, para os três métodos avaliados . . . . . . . . . . . . . . . .

6.9 As raízes dos erros quadráticos médios em cada método, calculados para cada pixel contido na região e mapeados. . . . . . . . . . . . . 58

6.10 Raízes dos erros quadráticos médios por tempo, para cada um dos métodos avaliados. . . . . . . . . . . . . . . . .

6.11 Raízes dos erros quadráticos médios pelo número de pontos faltantes numa imagem, para cada um dos métodos avaliados. . . . . . . . . . . . . 


\section{Lista de Tabelas}

5.1 Conjunto 1 - Erro quadrático médio da predição - Todas as observações . 44

5.2 Conjunto 2 - Erro quadrático médio da predição - Todas as observações . 44

5.3 Conjunto 1 - Erro quadrático médio - Observações mantidas no conjunto 44

5.4 Conjunto 2 - Erro quadrático médio - Observações mantidas no conjunto 45

5.5 Conjunto 1 - Erro quadrático médio da predição - Observações removidas

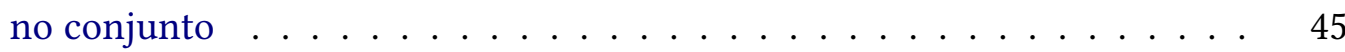

5.6 Conjunto 2 - Erro quadrático médio da predição - Observações removidas

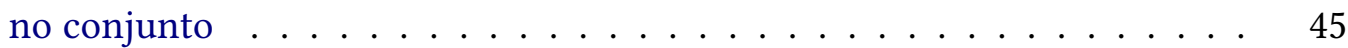

5.7 Tempo, em minutos, para a modelagem e a predição para o conjunto 1 (Sem sazonalidade) e o conjunto 2 (com sazonalidade) . . . . . . . . . 47

6.1 As coordenadas geográficas dos pontos que delimitam a área selecionada. $\quad 50$

6.2 Dados demográficos da área selecionada, por zona considerando os distritos incluídos por inteiro no mapa (IBGE, 2002). . . . . . . . . . . . . 52

6.3 Erros quadráticos médios considerando todas as observações. . . . . . . 57

6.4 Tempo, em minutos, para a modelagem e a predição para a aplicação. . . 59 



\section{Sumário}

1 Introdução 1

2 Conceitos Básicos 5

2.1 Processo Estocástico . . . . . . . . . . . . . . . . . . . 6

2.2 Processos Estocásticos Espaciais . . . . . . . . . . . . . . . 8

2.3 Processos Estocásticos Espaço-Temporais . . . . . . . . . . . . . . . . . . 10

2.4 Covariograma e Semivariograma Espaço-Temporal . . . . . . . . . . . . 11

2.5 Modelos Básicos para Dados Espaço-Temporais . . . . . . . . . . . . . . 12

3 Modelos de Efeitos Fixos e Aleatórios Espaço-Temporais $\quad 15$

3.1 Krigagem . . . . . . . . . . . . . . . . . . . . . . 15

3.2 Parametrização de Efeitos Aleatórios . . . . . . . . . . . . . . . . 18

3.3 Estimação dos Parâmetros com Algoritmo EM . . . . . . . . . . . . . . . 20

4 Modelo Hierárquico Baseado em Campos Aleatórios 23

4.1 Modelo Espaço-Temporal Hierárquico . . . . . . . . . . . . . . . . . . . 23

4.2 Obtenção da Matriz de Precisão Espacial . . . . . . . . . . . . . . . . 25

4.3 Estimação dos Parâmetros . . . . . . . . . . . . . . . . . 28

5 Estudos de Simulação

5.1 Descrição dos Processos de Simulação . . . . . . . . . . . . . . . . . . . . 31

5.2 Descrição dos Modelos Avaliados . . . . . . . . . . . . . . . . . . 34

5.3 Avaliação do Ajuste . . . . . . . . . . . . . . . . . . . . . . . . 36

5.4 Ilustração dos resultados . . . . . . . . . . . . . . . . . 36

5.5 Resultados . . . . . . . . . . . . . . . . . . . 43

5.6 Conclusões . . . . . . . . . . . . . . . . . . . 43

6 Aplicação $\quad 49$

6.1 Descrição dos Dados . . . . . . . . . . . . . . . . . . 49

6.2 Análise Descritiva . . . . . . . . . . . . . . . . . . . 52 
6.3 Predições . . . . . . . . . . . . . . . . . . . . . 55

7 Conclusões e Estudos Futuros

\section{Apêndices}

A Códigos 63

A.1 Simulação . . . . . . . . . . . . . . . . . . 63

A.2 Krigagem . . . . . . . . . . . . . . . . . . 64

A.3 Krigagem de Posto Fixo $(\mathrm{FRK}) \ldots \ldots \ldots \ldots$

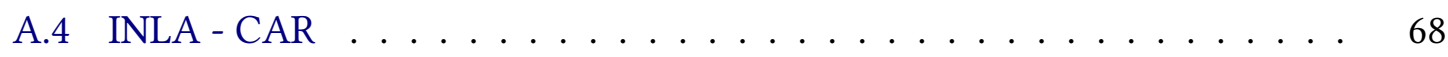

A.5 INLA - Triangulação . . . . . . . . . . . . . . . . . . . . 70

Referências para os pacotes utilizados . . . . . . . . . . . . . . . 73

$\begin{array}{ll}\text { Referências } & 75\end{array}$ 


\section{Capítulo 1}

\section{Introdução}

Informações provenientes de sensoriamento remoto se tornaram uma importante ferramenta no estudo científico de diversos fenômenos ocorrentes na Terra. Inúmeros campos de estudo se beneficiaram desse desenvolvimento tecnológico nas últimas décadas: a meteorologia, a geologia, a agricultura, o planejamento urbano, entre outros (Militino et al., 2018). A sempre crescente frota de satélites na órbita terrestre se tornou uma fonte constante de dados que enriquece pesquisas no mundo inteiro. A redução dos custos de armazenamento e distribuição de dados digitais facilitaram o acesso a essas informações. Órgãos como a NASA (National Aeronautics and Space Administration) e a ESA (European Space Agency) disponibilizam conjuntos de imagens de satélite e outras medições georreferenciadas de toda a superfície terrestre, coletados regularmente ao longo do tempo, sem custo para a comunidade científica.

Exemplos de medições oferecidas, além de imagens fotográficas, incluem: Índices de vegetação como o NDVI (Normalized Difference Vegetation Index) e o EVI (Enhanced Vegetation Index), gerados a partir dos valores de reflectância de bandas individuais na faixa espectral do azul, vermelho e infravermelho das imagens capturadas pelo sensor; a temperatura da superfície terrestre e a quantidade de $\mathrm{CO}_{2}$ na atmosfera, que podem ser estimadas a partir das medições de bandas de infravermelho; a topologia da superfície, obtida através de ondas de radar; dentre muitos outros (RichaRds e JiA, 2005). Esses conjuntos de dados são processados em aplicações que variam da detecção de incêndios florestais à classificação da ocupação do solo em áreas urbanas (FERREIRA, 2019).

À medida em que a tecnologia se desenvolve, aumenta a quantidade de informações coletadas por esses satélites. Sensores estão obtendo dados com maior resolução (um dado corresponde a uma área menor da superfície, aumentado o detalhamento da coleta) e com maior frequência. Lidar com esse grande volume de dados tem se mostrado um grande desafio, mesmo quando se tem disponível os mais avançados equipamentos e softwares de processamento de dados. O estudo de técnicas estatísticas para manipular grandes bancos de dados acompanha esse processo.

Ao mesmo tempo, problemas comuns em análises estatísticas continuam aparecendo. A presença de dados faltantes em conjuntos de dados oriundos de sensores remotos é 
recorrente e está relacionada não só à fenômenos climáticos como à presença de nuvens, como também a eventos provocados pela ação humana (as queimadas e a poluição urbana, por exemplo). A obstrução provocada por aerossóis de diversas origens na atmosfera também dificulta a medição de informações da superfície da Terra (WAN et al., 2015).

Muitas técnicas para análise de dados dependem de um conjunto de dados completo, e implementações em pacotes computacionais muitas vezes não são robustas para a presença de dados faltantes mesmo quando o trabalho teórico relacionado explora esse cenário. Inúmeras abordagens já foram estudadas para o preenchimento (ou imputação) desses dados. Substituição pela média, métodos de reamostragem como o bootstrap, modelos de regressão e métodos iterativos como o algoritmo de expectativa-maximização (EM). No contexto espacial, é comum o uso de métodos determinísticos como a inversa das distancias ponderadas, triangulações ou splines. No campo da geoestatística, um dos métodos mais consolidados é a Krigagem, uma forma de preditor linear baseado na proximidade do local onde se deseja interpolar o dado com localidades em que a informação foi observada.

Alguns trabalhos que lidam com a interpolação em conjuntos de dados referenciados espacialmente e temporalmente utilizam apenas dados de tempos anteriores ou posteriores, ignorando a informação contida em localidades próximas, ou apenas uma abordagem espacial como a Krigagem, ignorando a informação contida em tempos próximos ao que deseja interpolar (FERREIRA, 2019; GrÄLER et al., 2016).

Técnicas que combinam informações ao longo do espaço e do tempo para a interpolação têm sido desenvolvidas principalmente a partir dos anos 90. Vários autores propuseram diferentes tipos de filtros de Kalman espaço-temporais: HuAng e Cressie (1996), MARdia et al. (1998), Berke (1998), Meiring et al. (1998), Wikle e Cressie (1999).

Alguns métodos como a krigagem podem ser estendidos para incluir também os dados ao longo do tempo em conjunto com a informação espacial (CREsSIE, 1993; GrÄLER et al., 2016), no entanto, o aumento no volume de dados a serem processados pode inviabilizar o processo computacionalmente. Dentre as abordagens propostas para mitigar o problema estão o uso de agrupamentos de dados vizinhos e a limitação no uso de dados em cada predição (Gräler et al., 2016). Gribov e KrivoruchKo (2004) e Rivoirard e Romary (2011) propuseram distintos métodos de ponderação para localidades distantes. FurRer et al. (2006) propuseram uma abordagem para afilar (taper) a função de covariância entre as localidades para zero a partir de uma certa distância.

Cressie e Johannesson (2008) propuseram uma abordagem denominada Krigagem de Posto Fixo (do inglês Fixed Rank Kriging) em que se reduz a complexidade do processo de Krigagem modelando o conjunto de dados como modelo de efeitos aleatórios em que os dados são representados por uma combinação linear de funções-base com coeficientes aleatórios. Resultados satisfatórios foram obtidos para a predição linear em uma imagem de satélite de 193.000 pixels com o uso de apenas 396 funções-base. ZAMMIT-MANGion e CRESSIE (2021) apresentam uma extensão para o caso espaço-temporal.

Uma abordagem para a krigagem com Campos Aleatórios de Markov Gaussianos foi desenvolvida por Håvard RuE e HeLd (2005), relacionada a modelos espaciais formulados por Besag (1974), e estendida largamente devido ao trabalho de LindgRen, Håvard Rue et al. 
1 | INTRODUÇÃO

(2011), que estabeleceu relação para discretizar Campos Gaussianos através da equações estocásticas diferenciais parciais. O pacote computacional R-INLA (Håvard RUE, MARTINO et al., 2009) foi estendido para comportar essa abordagem; BLANGIARDO e CAMELETTI (2015) exploram a flexibilidade dessa técnica implementando modelos para diversos conjuntos de dados espaciais e espaço-temporais.

Neste trabalho, discutimos o uso de modelos de krigagem para a predição linear de dados em localidades e tempos onde se esperava a ocorrência de uma medição, mas não há medição disponível. Em particular, será dado enfoque aos métodos de Cressie e JohANNESSON (2008) e aos modelos baseados no trabalho de Håvard Rue, MARTino et al. (2009).

Este texto está organizado da seguinte forma: no Capítulo 2 revisamos alguns conceitos importantes para a compreensão dos métodos estudados num contexto espaço-temporal; no Capítulo 3 detalhamos métodos conhecidos de krigagem e apresentamos a Krigagem de Posto Fixo; no Capítulo 4 discutimos os conceitos por trás dos modelos de equações estocásticas parciais diferenciais no espaço e uma estratégia para o caso espaço-temporal; no Capítulo 5 comparamos as imputações dos métodos via simulação; no Capítulo 6 ilustramos esses modelos usando um conjunto de dados obtido por sensoriamento remoto: dados da temperatura de superfície terrestre em uma área da Região Metropolitana de São Paulo coletados entre 2009 e 2019. Algumas discussões e conclusões serão apresentadas no Capítulo 7. No Apêndice A, são apresentados os códigos utilizados no Capítulo 5. 



\section{Capítulo 2}

\section{Conceitos Básicos}

Dados espaciais consistem em medidas ou observações tomadas em locais específicos. Conjuntos de dados espaciais incluem, além dos valores de interesse, a localidade ou posição relativa onde os dados foram observados. localidades podem estar referenciadas pontualmente (por exemplo, por latitude e longitude) ou por regiões (por exemplo, por municípios definidos por seus limites geográficos). Em ambos os casos, as localidades podem estar espaçadas regularmente como em uma grade (por exemplo, em imagens completas de satélite) ou podem estar espaçadas com distâncias variáveis entre as observações. As localidades podem referenciar blocos contíguos de tamanhos iguais ou áreas das mais diversas formas. Muitos autores fazem distinção entre dados geoestatísticos (quando o domínio onde as observações são distribuídas em um espaço continuamente) e dados areais (quando o domínio é discretizado) (Kaluzny et al., 1998; Blangiardo e CAmeletti, 2015; Montero et al., 2015). No entanto, as ferramentas estatísticas são similares nos dois casos (Blangiardo e Cameletti, 2015; Cressie, Shi et al., 2010; Zimmermann, 2009; ZhaO e WALL, 2004). Uma importante característica de um conjunto de dados espaciais é que observações vizinhas ou próximas podem estar relacionadas entre si, possuindo informações umas sobre as outras.

Dados temporais consistem em medidas ou observações tomadas em tempos específicos. Conjuntos de dados temporais incluem, além dos valores de interesse, o tempo ou momento relativo onde os dados foram observados. Quando ordenados sequencialmente no tempo, esse conjunto de dados é normalmente chamado de série temporal. Um exemplo popular de série temporal é a medição diária de temperaturas máximas em um ponto de uma cidade. Esse exemplo, em que uma observação é feita num tempo específico é um caso de série dita discreta no tempo. Uma série temporal é dita contínua quando as observações são feitas continuamente no tempo, como registro de marés no porto de Santos, por exemplo (Morettin e Toloi, 2004). Nota-se a importância em séries temporais, analogamente aos conjuntos espaciais, do fato de que observações vizinhas ou próximas no tempo também podem estar relacionadas entre si.

Dados espaço-temporais consistem em medidas ou observações tomadas em locais e tempos específicos. Um conjunto de dados espaciais pode ser visto tanto como uma série temporal de conjuntos espaciais como um conjunto de séries temporais referenciadas no 
espaço.

Neste capítulo, apresentamos alguns conceitos básicos de séries e também alguns modelos simples para essa abordagem. Esses conceitos estão baseados principalmente em Morettin e Toloi (2004), Ehlers (2009) e Shumway e Stoffer (2017).

Também discutimos nesse capítulo séries no contexto espacial e espaço-temporal e apresentamos alguns conceitos importantes da área da geoestatística para a análise de dados no espaço e no espaço-tempo. As principais fontes são WikLE, Zammit-Mangion et al. (2019), Cressie e Wikle (2011) e Blangiardo e Cameletti (2015).

\subsection{Processo Estocástico}

Os modelos utilizados para descrever séries espaço-temporais são processos estocásticos, que são processos controlados por leis probabilísticas.

Definição 2.1. Um processo estocástico é uma família $Z=\{Z(t), t \in T, \omega \in \Omega\}$ e $Z(t)$ é uma variável aleatória definida num espaço de probabilidade $(\Omega, A, P)$ indexado por um indice $t$, onde $t$ varia no conjunto arbitrário $T$.

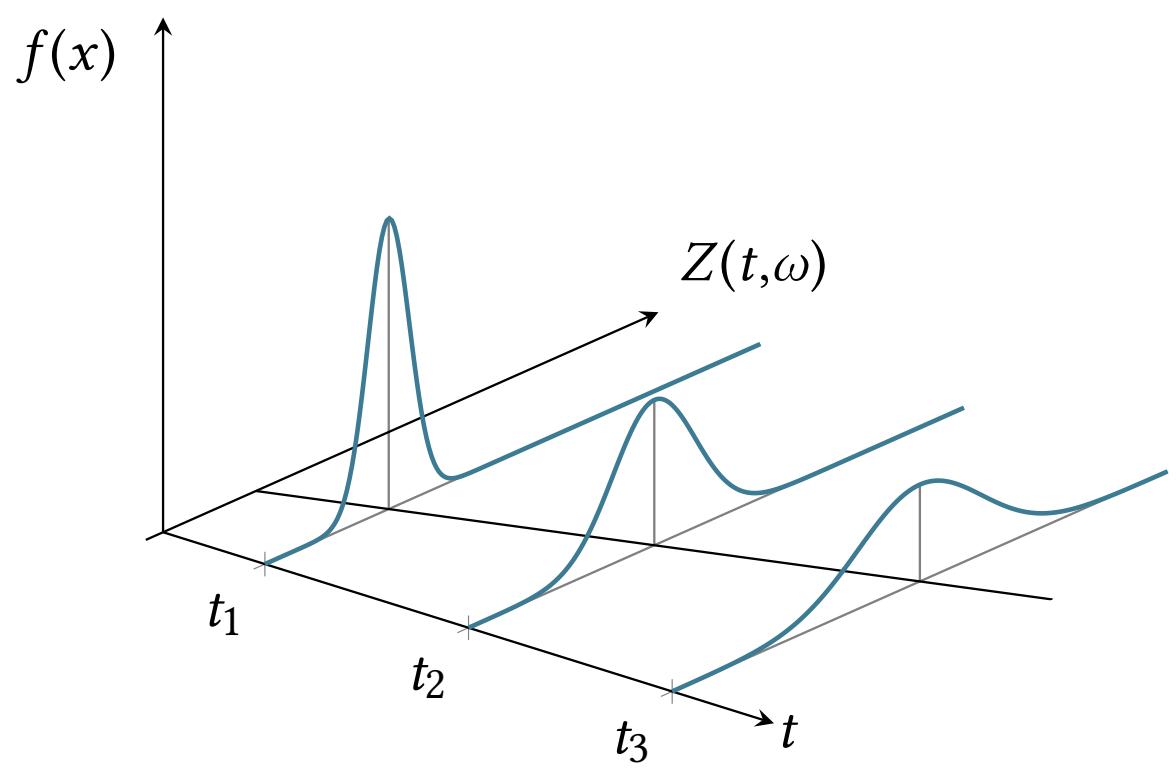

Figura 2.1: Processo estocástico, com cada variável $Z(t)$ definida em $(\Omega, A, P)$

Para cada $t \in T$, a função $Z(t, \cdot)$ é mensurável relativamente a A. Por outro lado, para cada $\omega \in \Omega$ fixado, teremos uma função $Z(\cdot, \omega)$ de $t: T \rightarrow \mathbb{R}$. Tal função é denominada trajetória, realização, função amostral do processo ou série.

Uma série temporal é uma realização particular do processo estocástico, denotada por $Z\left(t_{j}\right)$, , com tempo $t_{j}, j=1, \ldots n_{t}$.

Definição 2.2. A função de distribuição finito-dimensional do processo estocástico $Z=$ 
2.1 PROCESSO ESTOCÁSTICO

$\{Z(t), t \in T, \omega \in \Omega\}$ é definida como

$$
F\left(z_{1}, \ldots, z_{n_{t}} ; t_{1}, \ldots, t_{n_{t}}\right)=P\left(z\left(t_{1}\right) \leq z_{1}, \ldots, Z\left(t_{n_{t}} \leq z_{n_{t}}\right) .\right.
$$

Definição 2.3. As funções média e covariância de um processo $Z(t)$ são dadas por

$$
\begin{gathered}
E(Z(t))=\int_{-\infty}^{+\infty} z d F(z ; t)=\mu_{z}(t) ; \\
\operatorname{Cov}\left(Z\left(t_{1}\right), Z\left(t_{2}\right)\right)=\iint\left[z_{1}-\mu_{z}\left(t_{1}\right)\right]\left[z_{2}-\mu_{z}\left(t_{2}\right)\right] d F\left(z_{1}, z_{2}, t_{1}, t_{2}\right) \\
=E\left(Z\left(t_{1}\right), Z\left(t_{2}\right)\right)-E\left(Z\left(t_{1}\right)\right) E\left(Z\left(t_{2}\right)\right) \\
=C\left(t_{1}, t_{2}\right) .
\end{gathered}
$$

Se $t_{1}=t_{2}=t$, temos a variância do processo:

$$
\operatorname{Var}(Z(t))=\int\left[z-\mu_{z}(t)\right]^{2} d F(z, t)=E\left(Z(t)^{2}\right)-E(Z(t))^{2}=C(t)
$$

A função de autocorrelação $\rho\left(t_{1}, t_{2}\right)$ é definida como

$$
\rho\left(t_{1}, t_{2}\right)=\frac{\operatorname{Cov}\left(Z\left(t_{1}\right), Z\left(t_{2}\right)\right)}{\sqrt{\operatorname{Var}\left(Z\left(t_{1}\right)\right) \operatorname{Var}\left(Z\left(t_{2}\right)\right)}} .
$$

Uma importante característica a se considerar acerca de séries temporais é a estacionariedade. De acordo com MoretTin e Toloi (2004), um processo é estacionário se ele se desenvolver em seu domínio de modo que a escolha de um ponto inicial não muda a forma como o processo se desenvolve a partir desse ponto. Isto é, as características de $Z(t+\tau)$ são as mesmas de $Z(t)$, para qualquer $\tau \in \mathbb{R}$. É comum que se avalie três formas de estacionariedade: estrita (ou forte), de segunda ordem (ou fraca) e intrínseca.

Definição 2.4 (Estacionariedade). Considere o processo $\{Z(t), t \in T\}$, com média $\mu_{z}(t), \forall t \in$ $T$. Dizemos que o processo $\{Z(t), t \in T, \omega \in \Omega\}$ é

1. Estritamente estacionário se para quaisquer $t_{1}, \ldots, t_{n}$ com $t_{j} \in T, j=1, \ldots, n_{t}$ e $\tau \in \mathbb{R}$ com $t_{j}+\tau \in T, j=1, \ldots, n_{t}$, a distribuição do processo $\{Z(t), t \in T, \omega \in \Omega\}$ é a mesma do processo $\{Z(t), t+\tau \in \mathbb{R}, \omega \in \Omega\}$.

2. Fracamente estacionário se

- A média é constante, ou seja, $\mu(t)=\mu, \forall t \in T$;

- $E\left(Z^{2}(t)\right)<\infty, \forall t \in T$ 
- A covariância depende apenas de $\tau$, ou seja, $C(t, t+\tau)=C(\tau), \forall t \in T, \forall \tau \in \mathbb{R}$, $\operatorname{com} t+\tau \in \mathbb{R}$.

3. Intrinsecamente estacionário se

- $E(Z(t+\tau)-Z(t))=0, \quad \forall t \in T, \forall \tau \in \mathbb{R}, \operatorname{com} t+\tau \in \mathbb{R}$;

- $E(Z(t+\tau)-Z(t))^{2}=\operatorname{Var}(Z(t+\tau)-Z(t))=2 C(\tau), \quad \forall t \in T, \quad \forall \tau \in \mathbb{R}$, com $t+\tau \in \mathbb{R}$.

Outro caso importante a se considerar é o de quando a covariância diminui à medida em que a distância entre os tempos aumenta.

Definição 2.5 (Ergodicidade). Diz-se que um processo $\{Z(t), t \in T\}$ é ergódico se Cov $(Z(t+$ $\tau, Z(t)) \rightarrow 0$ quando $\tau \rightarrow \infty$.

Em alguns casos, como no caso de um processo Gaussiano, a estacionariedade fraca implica estacionariedade estrita.

Definição 2.6 (Processo Gaussiano). Diz-se que um processo $\{Z(t), t \in T\}$ é Gaussiano se, para qualquer sub-conjunto $t_{1}, \ldots, t_{n}$ de $T$, o conjunto das variáveis aleatórias $\left\{Z\left(t_{1}\right), \ldots, Z\left(t_{n}\right)\right\}$ tem distribuição Normal n-variada.

Definição 2.7 (Ruído Branco). Diz-se que um processo $\{Z(t), t \in T\}$ é ruído branco se as variáveis aleatórias $\left\{Z\left(t_{1}\right), \ldots, Z\left(t_{n}\right)\right\}$ são não correlacionadas, isto é,

$$
\operatorname{Cov}\left(X\left(t_{1}\right), X\left(t_{2}\right)\right)=0 \quad \forall \quad t_{1} \neq t_{2} ; t_{1}, t_{2} \in T .
$$

Tais processos serão estacionários se $E(Z(t))=\mu \forall t \in T$ e $\operatorname{Var}(Z(t))=\sigma^{2} \forall t \in T$. Se o conjunto das variáveis aleatórias $\left\{Z\left(t_{1}\right), \ldots, Z\left(t_{n}\right)\right\}$ tem distribuição Normal $n$-variada, temos o chamado ruído branco gaussiano.

\subsection{Processos Estocásticos Espaciais}

Os conceitos apresentados podem ser facilmente generalizados para o caso em que o parâmetro indexador do processo estocástico é um vetor e o conjunto a que esse valor pertence varia num espaço $d$-dimensional. Dessa forma, as mesmas técnicas apresentadas para o contexto temporal podem ser adaptadas para o contexto em que o processo se desenvolve no espaço. Seja $Z=\{Z(s), s \in \mathcal{D})\}$ um processo estocástico onde $\mathcal{D} \subset \mathbb{R}^{d}$ representa a localidade. Tipicamente se trabalha com aplicações onde $d=1,2$, ou 3. Neste trabalho será considerado o caso bidimensional $(\mathrm{d}=2)$.

Como no caso de uma dimensão, as funções média e covariância são dadas por $\mu_{z}(s) \mathrm{e}$ $\operatorname{Cov}\left(Z\left(s_{i}\right), Z\left(s_{k}\right)\right)$. O processo é dito estacionário se $\mu_{z}(\boldsymbol{s})=\mu$ para todo $s \in \mathcal{D}$ e $\operatorname{Cov}(Z(s, s+$ $\boldsymbol{h}))=C(\boldsymbol{h}), \boldsymbol{h} \in \mathbb{R}^{d}$.

Os conceitos adicionais mais importantes relacionados à covariância do contexto espacial está relacionado ao fato de que dois pontos possuem não apenas uma distância 
em si mas também uma direção. Neste trabalho, será considerada a distância euclidiana entre $s_{i}$ e $s_{k},\left\|s_{i}-s_{k}\right\|$, porém, em trabalhos em que se lida com superfícies mais extensas é comum o uso da distância geodésica ou da distância esférica.

Definição 2.8 (Ergodicidade). Diz-se que um processo $Z=\left\{Z(s), s \in \mathcal{D}_{s}\right\}$ é ergódico no espaço se $\operatorname{Cov}(Z(\boldsymbol{s}+\boldsymbol{h}), \boldsymbol{h})) \rightarrow 0$ quando $\|\boldsymbol{h}\| \rightarrow \infty$.

Processos ergódicos são importantes pois um processo espacial intrinsecamente estacionário será fracamente estacionário quando apresenta ergodicidade no espaço (SASSI, 2016). Ademais, a especificação de uma função de covariância fica mais simples nesse cenário.

Definição 2.9 (Isotropia). Diz-se que um processo $Z=\{Z(s), s \in \mathcal{D}\}$ é isotrópico se, para todo $s \in \mathcal{D}, C\left(Z\left(s_{i}\right), Z\left(s_{k}\right)\right)$ depende apenas da distância entre os pontos $s_{i}$ e $s_{k}$.

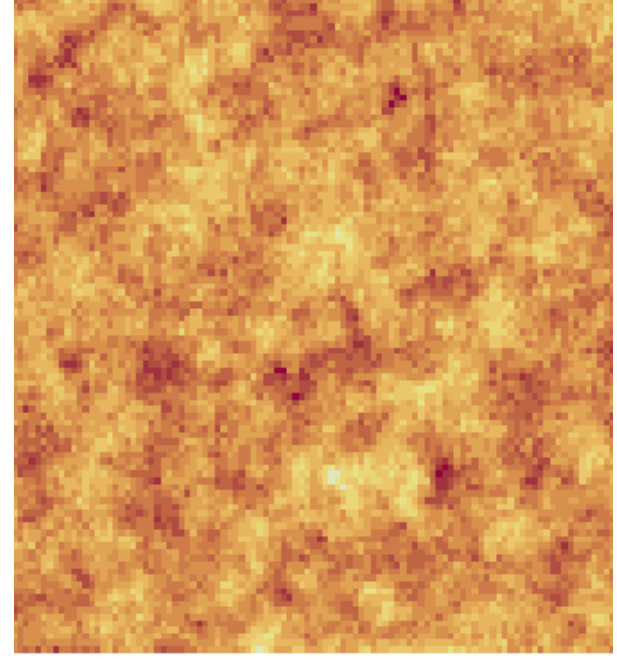

(a) Isotrópica

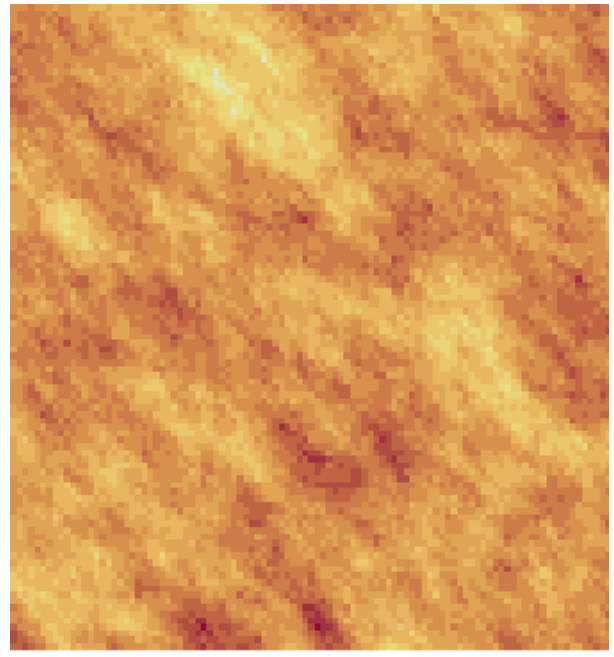

(b) Anisotrópica

Figura 2.2: Duas imagens simuladas exemplificando o conceito de isotropia.

Observa-se isotropia quando não pode-se notar uma direção específica no processo, mesmo quando se observa padrões. O caso em que se nota alguma direção é chamado anisotrópico.

Definição 2.10 (Campo Gaussiano). O processo $Z=\{Z(s), s \in \mathcal{D}\}$ é um campo Gaussiano se, para qualquer sub-conjunto $\boldsymbol{s}_{1}, \ldots, \boldsymbol{s}_{n}$ de $\mathcal{D}$, o conjunto das variáveis aleatórias $\left\{Z\left(s_{1}\right), \ldots, Z\left(s_{n}\right)\right\}$ tem distribuição Normal n-variada.

Uma versão discretizada do Campo Gaussiano, dada por $Z=\left\{Z\left(s_{i}\right), i=1, \ldots, n\right\}$ é chamada de campo aleatório Gaussiano de Markov (do inglês Random Markov Gaussian Field), abreviado como CAGM. Tais processos são muito usados para modelar a dependência entre dados observados no espaço. Seja $\boldsymbol{Z}$ um CAGM com média $\boldsymbol{\mu}$ e matriz de covariância $\Sigma$. A densidade de $Z$ é dada por

$$
f(z)=(2 \pi)^{-\frac{n}{2}}|\Sigma|^{-\frac{1}{2}} \exp \left(-\frac{1}{2}(z-\mu)^{\prime} \Sigma^{-1}(z-\mu)\right) .
$$


A inversa da matriz de covariância $\Sigma$ é chamada matriz de precisão e possui importante propriedade no contexto de campos Gaussianos de Markov, onde uma estrutura de vizinhança define que a distribuição condicional de $Z\left(s_{i}\right)$ depende de apenas alguns componentes de $Z$ (Håvard Rue e Held, 2005).

Teorema 2.1. Seja a matriz de precisão $Q=\Sigma^{-1}$. $Q_{i j}=0$ se e somente se $Z\left(s_{i}\right)$ e $Z\left(s_{j}\right)$, com $i \neq j$ são condicionalmente independentes, dados $Z\left(s_{-\{i, j\}}\right)$.

\subsection{Processos Estocásticos Espaço-Temporais}

Tal como apresentado na seção 2.2, também podemos facilmente considerar o caso em que o processo se desenvolve no espaço-tempo.

Definição 2.11. Um processo estocástico espaço-temporal é uma família $Z=\{Z(s, t), s \in$ $\left.\mathcal{D}_{s}, t \in \mathcal{D}_{t}, \omega \in \Omega\right\}$ e $Z(t)$ é uma variável aleatória definida num espaço de probabilidade $(\Omega, A, P)$ indexado pela localidade espacial s no domínio espacial $\mathcal{D}_{s}$ (um subconjunto do espaço euclidiano d-dimensional) e pelo tempo $t$ no domínio temporal $D_{t}$ (ao longo da reta real unidimensional).

Uma série espaço-temporal é uma realização particular do processo estocástico que denotamos por $Z(s, t)$, onde $s \in \mathcal{D}_{s}$ e $t \in \mathcal{D}_{t}$. Podemos entender tal processo estocástico como uma família de trajetórias em uma dada localidade e num dado tempo, da qual uma trajetória específica é observada.

Definição 2.12. Assumindo que temos as observações $\left\{Z\left(s_{i}, t_{j}\right)\right\}$ para as localidades espaciais $\left\{\boldsymbol{s}_{i}: i=1, \ldots, m\right\}$ e tempos $\left\{t_{j}: j=1, \ldots, T\right\}$. A média empírica espacial para a localidade $\boldsymbol{s}_{i}, \hat{\mu}_{z, s}\left(\boldsymbol{s}_{i}\right)$ é encontrada tomando a média amostral no tempo:

$$
\hat{\mu}_{z, s}\left(s_{i}\right)=\frac{1}{T} \sum_{j=1}^{T} Z\left(s_{i}, t_{j}\right)
$$

Definição 2.13. A média empírica temporal para o tempo $t_{j}, \hat{\mu}_{z, t}\left(t_{j}\right)$ é encontrada tomando a média amostral no espaço:

$$
\hat{\mu}_{z, t}\left(t_{j}\right)=\frac{1}{m} \sum_{i=1}^{m} Z\left(s_{i}, t_{j}\right)
$$

Definição 2.14. A covariância empírica de lag $\tau$ entre as localidades espaciais $\boldsymbol{s}_{i}$ e $\boldsymbol{s}_{k}$ é dada por

$$
\hat{C}_{z}(\tau)\left(\boldsymbol{s}_{i}, \boldsymbol{s}_{k}\right) \equiv \frac{1}{T-\tau} \sum_{j=\tau+1}^{T}\left(Z\left(\boldsymbol{s}_{i}, t_{j}\right)-\hat{\mu}_{z, s}\left(\boldsymbol{s}_{i}\right)\right)\left(Z\left(\boldsymbol{s}_{k}, t_{j}-\tau\right)-\hat{\mu}_{z, s}\left(\boldsymbol{s}_{k}\right)\right),
$$

para $\tau=0,1, \ldots, T-1$, que é chamada de covariância espacial empírica de lag $\tau$.

É frequentemente útil considerar a matriz $m \times m$ de covariância espacial empírica de 
$\operatorname{lag} \tau, \hat{C}_{z}^{(\tau)}$, em que o $(i, k)$-ésimo elemento é dado pela equação acima. Alternativamente isso pode ser calculado como

$$
\hat{\boldsymbol{C}}_{z}(\tau) \equiv \frac{1}{T-\tau} \sum_{j=\tau+1}^{T}\left(Z_{t j}-\hat{\boldsymbol{\mu}}_{z, \mathrm{~s}}\right)\left(Z_{t_{j}-\tau}-\hat{\boldsymbol{\mu}}_{z, \mathrm{~s}}\right)^{\prime} ; \tau=0,1, \ldots, T-1,
$$

para $\tau=0,1, \ldots, T-1$, que é chamada de covariância espacial empírica de lag- $\tau$.

\subsection{Covariograma e Semivariograma Espaço-Temporal}

Nos procedimentos em que se deseja realizar predição ótima, é necessário avaliar a dependência no espaço e no tempo conjuntamente. Nesse caso, precisamos caracterizar a variabilidade nos dados espaço-temporais como funções dos específicos lags no espaço e no tempo. Na definição que segue, consideramos que temos estacionariedade fixados os espaços. Isto é, a média $\mu_{s, t}$ pode depender de $s$ mas não depende de $t$ e a variância depende apenas das diferenças nos lags tanto no espaço quanto no tempo.

Definição 2.15 (Covariograma). O covariograma espaço-temporal para os lags $\boldsymbol{h}$ e $\tau$ é dado por

$$
\hat{\boldsymbol{C}}_{z}(\boldsymbol{h}, \tau)=\frac{1}{\left|N_{s}(\boldsymbol{h})\right|} \frac{1}{\left|N_{t}(\tau)\right|} \sum_{\boldsymbol{s}_{i}, s_{k} \in N_{s}(\boldsymbol{h})} \sum_{t_{j}, t_{l} \in N_{t}(\tau)}\left(Z\left(\boldsymbol{s}_{i}, t_{j}\right)-\hat{\mu}_{z, s}\left(\boldsymbol{s}_{i}\right)\right)\left(Z\left(\boldsymbol{s}_{k}, t_{l}\right)-\hat{\mu}_{z, s}\left(\boldsymbol{s}_{k}\right)\right),
$$

onde $N_{s}(\boldsymbol{h})$ se refere aos pares de localidades espaciais com lag espacial menor ou igual a $\|\boldsymbol{h}\|$, $N_{t}(\tau)$ se refere aos pares de pontos no tempo com lag temporal menor ou igual a $|\tau|$, e $N(\cdot)$ se refere ao número de elementos em $N(\cdot)$. Sob isotropia, o lag é considerado uma mera função da distância, $h=\|\mathbf{h}\|$.

No campo da geoestatística, historicamente se usa para avaliações de dependência espacial e espaço-temporal uma outra definição, fortemente relacionada ao covariograma, chamada semivariograma.

Definição 2.16 (Semivariograma). O semivariograma espaço-temporal é dado por

$$
\gamma_{z}\left(s_{i}, s_{k}, t_{j}, t_{l}\right) \equiv \frac{1}{2} \operatorname{Var}\left(Z\left(s_{i}, t_{j}\right)-Z\left(s_{k}, t_{l}\right)\right)
$$

No caso em que a covariância depende apenas dos deslocamentos no espaço $\boldsymbol{h}$ e das diferenças no tempo $\tau$, essa relação pode ser escrita como

$$
\begin{aligned}
\gamma_{z}(\boldsymbol{h}, \tau) \equiv \frac{1}{2} \operatorname{Var}(Z(\boldsymbol{s}+\boldsymbol{h}, t+\tau)-Z(\boldsymbol{s}, t)) & =C_{z}(\mathbf{0}, 0)-\operatorname{Cov}(Z(\boldsymbol{s}+\boldsymbol{h}, t+\tau), Z(\boldsymbol{s}, t)) \\
& =C_{z}(\mathbf{0}, 0)-C_{z}(\boldsymbol{h}, \tau)
\end{aligned}
$$


em que $\boldsymbol{h}=\boldsymbol{s}_{k}-\boldsymbol{s}_{i}$ é o lag espacial e $\tau=t_{j}-t_{l}$ é o lag temporal.

Quando o processo espaço-temporal é ergódico, então o semivariograma é geralmente caracterizado pelo efeito nugget (pepita), a sela e a sela parcial. O efeito nugget é dado por $\gamma_{z}(\boldsymbol{h}, \tau)$ quando $\boldsymbol{h} \rightarrow \mathbf{0}$ e $\tau \rightarrow 0$, enquanto a sela é $\gamma_{z}(\boldsymbol{h}, \tau)$ quando $\boldsymbol{h} \rightarrow \infty$ e $\tau \rightarrow \infty$ . A sela parcial é a diferença entre a sela e o efeito pepita. O diagrama abaixo mostra os componentes do semivariograma como função da distância espacial $\|\boldsymbol{h}\|$. Sob essas condições, podemos escrever $\hat{\gamma}_{z}(\boldsymbol{h}, \tau)=\hat{\boldsymbol{C}}_{z}(\mathbf{0}, 0)-\hat{\boldsymbol{C}}_{z}(\boldsymbol{h}, \tau)$.

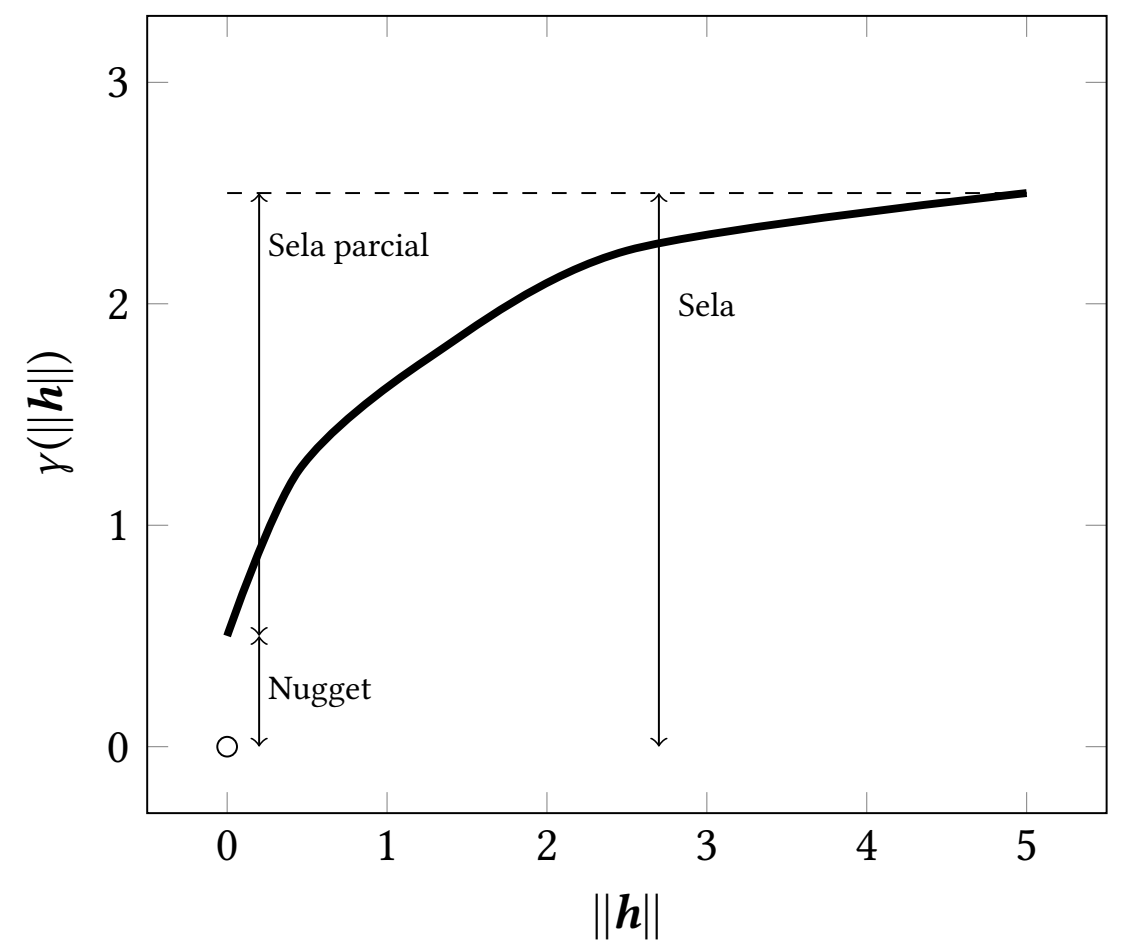

Figura 2.3: Componentes de um semivariograma como função da distância $\|\boldsymbol{h}\|$.

\subsection{Modelos Básicos para Dados Espaço-Temporais}

Podemos usar modelos básicos de regressão para a obtenção de previsões para localidades no espaço-tempo dado um número de observações encontradas. Em tais modelos, são incluídas como covariáveis as localidades no espaço e no tempo. Uma configuração típica considera uma covariável $X_{(t)}(s, t)$ como o tempo em que a observação foi coletada e $X_{(s)}(s, t)$ um indicador de qual região a observação representa. As localidades também são comumente representadas por um par $X_{(l a t)}(s, t), X_{(l o n)}(s, t)$, representando a latitude e a longitude das observações. Outras covariáveis associadas às medições podem ser incluídas na modelagem. O modelo então é formulado para os tempos discretos $t_{j}: j=1, \ldots, T$ e para as localidades espaciais $s_{i}: i=1, \ldots, m_{j}$ como

$$
Z\left(s_{i}, t_{j}\right)=\beta_{0}+\beta_{1} X_{1}\left(s_{i}, t_{j}\right)+\ldots+\beta_{p} X_{p}\left(s_{i}, t_{j}\right)+e\left(s_{i}, t_{j}\right),
$$


em que $\beta_{0}$ é o intercepto e $\beta_{k},(k>0)$ é um coeficiente de regressão associado com $X_{k}\left(s_{i}, t_{j}\right)$, a $k$-ésima covariável na localidade espacial $s_{i}$ e temporal $t_{j}$. Nesse modelo, assume-se que as observações são independentes e identicamente distribuídas tais que $e\left(s_{i}, t_{j}\right) \sim N\left(0, \sigma^{2}\right)$ para todo $s_{i}, t_{j}$.

Os coeficientes $\beta_{0}, \ldots ., \beta_{p}$ podem ser estimados pela minimização da soma dos resíduos, dada por

$$
S Q R=\sum_{j=1}^{T} \sum_{i=1}^{m_{j}}\left(Z\left(\boldsymbol{s}_{i}, t_{j}\right)-\hat{Z}\left(\boldsymbol{s}_{i}, t_{j}\right)\right)^{2}
$$

Chamando esses estimadores de $\beta_{0}, \ldots, \beta_{p}$, escrevemos $\hat{Z}\left(s_{i}, t_{j}\right)=\hat{\beta}_{0}+\hat{\beta}_{1} X_{1}\left(s_{i}, t_{j}\right)+\ldots+$ $\hat{\beta}_{1} X_{p}\left(s_{i}, t_{j}\right)$. Também estima-se a variância como

$$
\hat{\sigma}^{2}=\frac{S Q R}{\left(\sum_{j=1}^{T} m_{j}-p-1 .\right)} .
$$

Observa-se que essa formulação permite encontrar predições não-viesadas e consistentes para qualquer ponto não observado $\left(s_{0}, t_{0}\right)$ desde que $t_{0} \in T$ e $s_{0} \in \mathcal{D}$ (Wikle, ZammitMANGion et al., 2019). A variância da estimativa é dada por $\hat{\sigma}_{e}^{2}\left(X\left(s_{i}, t_{j}\right)\left(X^{\prime} X\right)^{-1} X^{\prime} z\left(s_{i}, t_{j}\right)\right)$. Se está sendo predito um ponto não observado $Z\left(s_{0}, t_{0}\right)$, então a variância da predição é dada por $\hat{\sigma}_{e}^{2}\left(1+X\left(s_{0}, t_{0}\right)\left(X^{\prime} X\right)^{-1} X^{\prime} z\left(s_{0}, t_{0}\right)\right)$.

No entanto, é muito comum que esse tipo de modelo não explique toda a variabilidade espaço-temporal. Isso decorre do fato de que as estruturas de correlação espaciais e temporais estão sendo ignoradas na suposição de que as observações são independentes entre si. Esse fato é facilmente verificável através de uma análise dos resíduos ou de um teste de independência como Durbin-Watson (Kutner et al., 2005). Nessas condições, os erros padrões e os erros padrões das predições são viesados (subestimados), dando uma falsa impressão do quão boas as estimativas e as predições realmente são. (WIKLE, ZAMMit-MANGion et al., 2019)

A fim de tratar esse tipo de dependência, um dos métodos mais utilizados é baseado em uma regressão envolvendo valores próximos.

Definição 2.17. Um processo $Y=\left\{Y_{t}, t \in \mathcal{D}_{t}, \omega \in \Omega\right\}$ é definido autorregressivo de ordem $p, A R(p)$, por

$$
Y_{t}=a_{1} Y_{t-1}+a_{2} Y_{t-2}+\ldots+a_{p} Y_{t-p}+\varepsilon_{t},
$$

$\operatorname{com} \varepsilon_{t}$ sendo ruído branco de média zero e variância $\sigma_{\omega}^{2}$.

No caso em que $p=1$, temos o modelo $\operatorname{AR}(1)$ :

$$
Y_{t}=a_{1} Y_{t-1}+\varepsilon_{t}
$$


Quando $|a|<1$ e $\operatorname{Var}\left(Y_{t}\right)<\infty$ para todo $t \in T$, o processo $\mathrm{AR}(1)$ é estacionário com média $E\left(Y_{t}\right)=0$, função de covariância $C(h)=\frac{\sigma_{\varepsilon} a^{h}}{1-a^{2}}$ e função de correlação $\rho(h)=a^{h}, h>0$. Pode-se mostrar que, no caso em que $\varepsilon \sim N\left(0, \sigma_{\varepsilon}^{2}\right)$, um estimador ótimo (de mínimos quadrados) para $a$ é $\hat{a}=\hat{\rho}=\frac{\sum_{i=2}^{T} y_{i} y_{i-1}}{\sum_{i=1}^{T} y_{i}^{2}}, \operatorname{com} \hat{\sigma}_{\varepsilon}^{2} \frac{1}{T-1} \sum_{t=2}^{T}\left(y_{t}-\hat{a} y_{t-1}\right)^{2}$.

No caso espacial, Whittle (1954) definiu uma classe de modelos que chamou Autorregressiva Espacial (SAR) dada por

$$
Y\left(s_{i}\right)=a w\left(s_{i}\right)^{\prime} Y+\varepsilon, \quad \varepsilon \sim N\left(0, \sigma_{\varepsilon}^{2}\right)
$$

em que $w\left(s_{i}\right)$ é um vetor de dimensão $m$ que associa ao local $s_{i}$ pesos de acordo com a distância entre as localidades $s_{i}$ e $s_{k}, k=1, \ldots, m$. Por exemplo, pode-se adotar peso 1 se a $k$-ésima localidade for vizinha de $s_{i}$ e peso 0 caso contrário.

Uma outra classe de modelos, devida a Besag (1974) e chamada Autorregressiva condicional (CAR), é definida condicionalmente por

$$
y\left(s_{i}\right) \mid y \sim N\left(a \sum_{j=1}^{m} w_{i j}, \frac{1}{\left|N\left(s_{i}\right)\right|}\right),
$$

onde $N\left(s_{i}\right)$ é o conjunto de pontos vizinhos de $\boldsymbol{s}_{i}$.

Whittle (1954) mostrou imediatamente que os resíduos do modelo SAR estão correlacionados com os valores vizinhos e que os estimadores de mínimos quadrados dos parâmetros do modelo SAR são inconsistentes. O modelo CAR minimiza o erro quadrático médio de previsão para $y\left(\boldsymbol{s}_{\boldsymbol{i}}\right)$ (CRESSIE, 1993). KISSLING e CARL (2008) obteve modelos SAR mistos, Ver Hoef et al. (2018) discute em mais detalhes os modelos CAR.

Para o caso espaço-temporal, destacamos a classe de modelos STARMA (Autorregressivo e média móvel espaço-temporal) (Cressie, 1993), um caso especial dos modelos VARMA definidos por (Box e JENKINS, 2015), desenvolvidos por ClifF e ORD (1975) e mais detalhadamente por Pfeifer e Deutsch (1980). Para mais detalhes sobre essa classe de modelos, consultar Jin (2017). 


\section{Capítulo 3}

\section{Modelos de Efeitos Fixos e Aleatórios Espaço-Temporais}

No Capítulo 2, definimos as séries espaço-temporais e apresentamos alguns conceitos elementares para se trabalhar com modelos para esses conjuntos de dados, como a covariância espacial e a isotropia. Também apresentamos uma estratégia básica para modelagem através de regressão linear, apontando os problemas em que essa abordagem incorre.

Neste capítulo, será apresentado o método de krigagem (MATHERON, 1963), amplamente usado para predição linear (e por consequência, para a interpolação de dados faltantes), com boas propriedades e possibilidade da quantificação da incerteza para a predição. Apresentamos a extensão para o caso espaço-temporal com base nos textos de CRESSIE e Wikle (2011) e Wikle, Zammit-Mangion et al. (2019). Em seguida, apresentamos a abordagem de Zammit-Mangion e Cressie (2021), baseada em Cressie e Johannesson (2008) e denominada Krigagem de Posto Fixo (Fixed Rank Kriging ou FRK), para viabilizar a implementação computacional de um método de Krigagem em face do aumento na quantidade de observações no espaço-tempo.

\subsection{Krigagem}

Seja o processo espaço-temporal $\left\{Y(s, t): s \in \mathcal{D} \subset \mathbb{R}^{d}, t \in\{1,2 \ldots\}\right\}$, com observações dadas por

$$
Z(s, t)=Y(s, t)+\epsilon(s, t),
$$

onde $\left\{\epsilon(s, t): s \in D \subset \mathbb{R}^{d}, t \in\{1,2 \ldots\}\right\}$ representa o erro de medição independente de $Y(\cdot ; \cdot)$ com variância $\sigma_{\epsilon}^{2}$. Assumimos ainda que $E(\epsilon(s, t) \epsilon(\boldsymbol{r}, u))=0$ exceto quando $\boldsymbol{s}=\boldsymbol{r}$ e $t=u$, e assumindo que $Y(s, t)$ tem a seguinte estrutura:

$$
Y(s, t)=\mu(s, t)+\lambda(s, t),
$$


na qual $\mu(\cdot, \cdot)$ é uma função de média (espaço-temporal) determinística, ou tendência, e $\lambda(s, t)$ representa um processo aleatório com dependência espacial e temporal. Um modelo comum para $\mu$ é,$\mu(\cdot ; \cdot)=X(\cdot ; \cdot)^{\prime} \boldsymbol{\beta} \operatorname{com} X \equiv\left[x\left(s_{i j}, t_{j}\right)^{\prime}: i=1, \ldots, m_{j} ; j=1, \ldots, T\right]$ como matriz de $\sum_{j=1}^{T} m_{j}$ observações e $(p-1)$ covariáveis e $\boldsymbol{\beta}$ vetor $\mathrm{p}$-dimensional de parâmetros.

Podemos escrever o modelo dos dados em termos de vetores,

$$
Z=\mu+\lambda+\epsilon
$$

$\operatorname{com} \operatorname{Cov}(Y) \equiv C_{y}=C_{\lambda}, \operatorname{Cov}(\epsilon)=C_{\epsilon}$ e $\operatorname{Cov}(Z) \equiv C_{z}=C_{y}+C_{\epsilon}$.

Definindo $\boldsymbol{c}_{0}^{\prime} \equiv \operatorname{Cov}\left(Y\left(s_{0}, t_{0}\right), Z\right), c_{0,0} \equiv \operatorname{Var}\left(Y\left(s_{0}, t_{0}\right)\right)$, considere a seguinte distribuição Gaussiana conjunta,

$$
\left[\begin{array}{c}
Y\left(\boldsymbol{s}_{0}, t_{0}\right) \\
\boldsymbol{Z}
\end{array}\right] \sim N\left(\left[\begin{array}{c}
\boldsymbol{x}\left(\boldsymbol{s}_{0}, t_{0}\right)^{\prime} \\
\boldsymbol{X}
\end{array}\right] \boldsymbol{\beta},\left[\begin{array}{cc}
c_{0,0} & \boldsymbol{c}_{0}^{\prime} \\
\boldsymbol{c}_{0} & C_{z}
\end{array}\right]\right)
$$

Aplicando resultados conhecidos da distribuição Gaussiana conjunta (SEARLE, 1982), obtemos a distribuição condicional

$$
Y\left(s_{0}, t_{0}\right) \mid Z \sim N\left(x\left(s_{0}, t_{0}\right)^{\prime} \boldsymbol{\beta}+\boldsymbol{c}_{0}^{\prime} C_{z}^{-1}(Z-X \boldsymbol{\beta}), c_{0,0}-\boldsymbol{c}_{0}^{\prime} C_{z}^{-1} \boldsymbol{c}_{0}\right),
$$

para a qual a média é o preditor da krigagem simples espaço-temporal,

$$
\hat{Y}\left(s_{0}, t_{0}\right)=x\left(s_{0}, t_{0}\right)^{\prime} \boldsymbol{\beta}+\boldsymbol{c}_{0}^{\prime} \boldsymbol{C}_{z}^{-1}(Z-X \boldsymbol{\beta}),
$$

com erro padrão da predição sendo a raiz quadrada da variância da krigagem espaçotemporal simples,

$$
\sigma_{Y}^{2}\left(s_{0}, t_{0}\right)=c_{0,0}-c_{0}^{\prime} C_{z}^{-1} c_{0}
$$

$\mathrm{Na}$ maior parte dos problemas, os parâmetros $\boldsymbol{\beta}$ são desconhecidos e precisam ser estimados. Nesses casos, o estimador de mínimos quadrados generalizados para $\boldsymbol{\beta}$ é obtido por

$$
\hat{\boldsymbol{\beta}}_{\text {mqg }} \equiv\left(X^{\prime} C_{z}^{-1} X\right)^{-1}\left(X^{\prime} C_{z}^{-1} Z\right),
$$

e o preditor linear não-viesado ótimo, ou preditor da krigagem espaço-temporal universal para $Y\left(s_{0}, t_{0}\right)$ é

$$
\hat{Y}\left(\boldsymbol{s}_{0}, t_{0}\right)=\boldsymbol{x}\left(\boldsymbol{s}_{0}, t_{0}\right)^{\prime} \hat{\boldsymbol{\beta}}_{m q g}+\boldsymbol{c}_{0}^{\prime} \boldsymbol{C}_{z}^{-1}\left(\boldsymbol{Z}-\boldsymbol{X} \hat{\boldsymbol{\beta}}_{m q g}\right),
$$

com o erro padrão da predição da krigagem espaço-temporal universal dado pela raiz quadrada da variância,

$$
\sigma_{Y}^{2}\left(s_{0}, t_{0}\right)=c_{0,0}-c_{0}^{\prime} C_{z}^{-1} c_{0}+\left(x\left(s_{0}, t_{0}\right)-X^{\prime} C_{z}^{-1} c_{0}\right)^{\prime}\left(X^{\prime} C_{z}^{-1} X\right)^{-1}\left(X^{\prime} C_{z}^{-1} c_{0}\right)
$$


Um dos maiores desafios em estatística espacial está no entendimento das variâncias e covariâncias que compõem a matriz $C_{z}=C_{y}+C_{\epsilon}$, uma vez que estas raramente são conhecidas previamente. Uma solução amplamente adotada está em definir uma função de covariância com parâmetros $\boldsymbol{\theta}$ (normalmente relacionados ao espaço e ao tempo) e estimar $C_{z}$ através de métodos de máxima verossimilhança.

Seja uma função de covariância espaço-temporal não-negativa definida dada por

$$
c_{*}\left(s, s^{\prime} ; t, t^{\prime}\right) \equiv \operatorname{Cov}\left(Y(s, t), Y\left(s^{\prime}, t^{\prime}\right)\right)
$$

Muitos modelos de Krigagem pressupõem que o processo aleatório espaço-temporal é estacionário de segunda ordem ou fracamente estacionário. Estendendo a Definição 2.4 para o caso espaço-temporal, um processo aleatório é fracamente estacionário se ele possui valor esperado constante $\mu$ e função de covariância que pode ser expressa em termos de lags espaço-temporais:

$$
c_{*}\left(s, s^{\prime} ; t, t^{\prime}\right)=C\left(s-s^{\prime}, t-t^{\prime}\right)=C(\boldsymbol{h}, r)
$$

Uma classe importante de funções de covariância espaço-temporais é a das funções separáveis, que podem ser escritas como

$$
C(\boldsymbol{h}, r) \equiv C^{(s)}(\boldsymbol{h}) \cdot C^{(t)}(r)
$$

e são válidas se tanto a função de covariância espacial, $C^{(s)}(\boldsymbol{h})$, e a função de covariância temporal, $C^{(t)}(r)$, são positivas definidas.

Uma consequência da separabilidade é que a função de correlação espaço-temporal também pode ser escrita como

$$
\rho(\boldsymbol{h}, r) \equiv \frac{C(\boldsymbol{h}, r)}{C(\mathbf{0}, 0)}=\frac{C^{(s)}(\boldsymbol{h}) \cdot C^{(t)}(r)}{C^{(s)}(\mathbf{0}) \cdot C^{(t)}(0)}=\rho^{(s)}(\boldsymbol{h}, 0) \cdot \rho^{(t)}(\mathbf{0}, r),
$$

onde $\rho^{(s)}(\boldsymbol{h}, 0)$ é a função de correlação marginal espacial e $\rho^{(t)}(\mathbf{0}, r)$ é a função de correlação marginal temporal. Dessa forma é possível obter as funções de correlação espaço-temporal conjunta a partir das funções de correlação marginais. Assim, é possível escrever $C_{z}=$ $C_{z}^{(t)} \otimes C_{z}^{(s)}$.

Apesar das vantagens computacionais e de interpretação de uma função de covariância separável, nem sempre é razoável adotar uma função dessa classe para modelar processos observados na natureza. Outros métodos para construção de função de correlações espaçotemporais foram explorados por CRESSIE e HUANG (1999), que construíram uma função nãoseparável válida ao lançar o problema no domínio espectral. Uma abordagem envolvendo a solução de equações diferenciais parciais estocásticas será discutida no Capítulo 4 desse trabalho.

Estabelecer uma função de covariância espaço-temporal válida pode ser muito compli- 
cado devido à dificuldade de se entender a forma como espaço e tempo interagem e afetam o processo analisado. Ademais, como a solução das equações de krigagem envolvem a inversão da matriz de covariâncias $C_{z}$, de ordem $n \times n$, temos um problema com custo computacional de $O\left(n^{3}\right)$. Uma maneira de trabalhar essa questão é através de uma modelagem condicional que se beneficia de especificações condicionais. CRESSIE e JoHANNESSON (2008) propuseram um método para obter o melhor preditor linear não-viesado através de modelo de efeitos mistos linear, de modo que se possa construir a dependência espaço-temporal de uma maneira que é sempre válida e oferece vantagens computacionais. Embora o método tenha sido desenvolvido apenas para modelos espaciais, Zammit-MAngion e Cressie (2021) o estenderam para o caso espaço-temporal.

\subsection{Parametrização de Efeitos Aleatórios}

Assumindo o mesmo modelo de dados (3.3), podemos reescrever o processo (3.2) em termos de efeitos fixos e aleatórios,

$$
Y(s, t)=\mu(s, t)+\lambda(s, t)=x(s, t)^{\prime} \boldsymbol{\beta}+\sum_{l=1}^{r} \phi_{l}(s, t) \alpha_{l}+\xi(s, t),
$$

ou em forma matricial,

$$
Y=X \beta+\Phi \alpha+\xi
$$

onde

- $\phi(\cdot) \equiv\left(\phi_{1}(\cdot), \ldots, \phi_{r}(\cdot)\right)^{\prime}$ é um vetor pré-especificado de bases escolhidas a fim de reconstruir adequadamente as realizações de $Y(\cdot)$;

- $\alpha_{i}$ são efeitos aleatórios, que resultam da decomposição do processo espaço-temporal $\lambda(s, t)$ e um termo residual $\xi$;

- $\xi$ representa efeitos aleatórios de pequena escala espaço-temporais que não foram capturados pelas bases espaço-temporais e definidos como quase não-correlacionados (Zammit-Mangion e Cressie, 2021). Cressie, Shi et al. (2010) argumentam que $\xi$ é análogo ao chamado efeito nugget observado no semivariograma (Figura 2.3). Nesse trabalho, consideramos $\operatorname{Cov}(\xi)=C_{\xi}=\sigma_{\xi}^{2} V_{\xi}$, $\operatorname{com} V_{\xi}$ uma matriz diagonal $n \times n$.

Então, em (3.12) apenas decompomos o processo aleatório espaço-temporal, $\lambda(s, t)$, em uma combinação linear de efeitos aleatórios e um termo para efeitos que não são adequadamente modelados pelas funções-base.

Assumindo $\boldsymbol{\alpha} \sim N\left(\mathbf{0}, C_{\alpha}\right)$, temos que a variância de pequena escala em (3.12) é modelada como um processo espaço-temporal Gaussiano. Considere a representação condicional de modelos lineares de efeitos mistos (LAIRD e WARE, 1982):

$$
Z \mid \boldsymbol{\alpha} \sim N\left(X \boldsymbol{\beta}+\Phi \boldsymbol{\alpha}, C_{\xi}\right),
$$

com função de densidade marginal de $Z$ dada por: 


$$
f_{Z}(z)=\int f_{Z \mid \alpha}(z \mid \boldsymbol{\alpha}) f(\boldsymbol{\alpha}) d \boldsymbol{\alpha}
$$

Então,

$$
\begin{gathered}
E(Z)=E_{\alpha}\{E(Z \mid \boldsymbol{\alpha})\}=E_{\alpha}\{X \boldsymbol{\beta}+\boldsymbol{\Phi} \boldsymbol{\alpha}\}=X \boldsymbol{\beta} \\
\operatorname{Var}(Z)=\operatorname{Var}_{\alpha}\{E(Z \mid \boldsymbol{\alpha})\}+E_{\alpha}\{\operatorname{Var}(Z \mid \boldsymbol{\alpha})\}=\boldsymbol{\Phi} C_{\alpha} \boldsymbol{\Phi}^{\prime}+C_{\xi} .
\end{gathered}
$$

Uma vez que $Z$ é uma combinação linear de variáveis aleatórias com distribuição normal, $Z$ também tem distribuição normal com

$$
Z \sim N\left(X \beta, \Phi C_{\alpha} \Phi^{\prime}+C_{\xi}\right)
$$

Wikle, ZAMmit-MANGion et al. (2019) argumentam que essa abordagem, induzindo uma estrutura de covariância mais complicada na distribuição marginal (com matriz de covariância $\Phi C_{\alpha} \Phi^{\prime}+C_{\xi}$ ) do que na distribuição condicional ( com matriz de covariância $C_{\xi}$ ), simplifica o processo de modelagem uma vez que é mais fácil modelar médias do que modelar covariâncias. Além disso, os efeitos aleatórios $\boldsymbol{\alpha}$ não estão indexados no tempo e no espaço, facilitando a especificação de um modelo para eles. Por exemplo, podemos especificar uma matriz de covariância para descrever a dependência entre os efeitos $\boldsymbol{\alpha}$, ao invés de uma função de covariância.

Outro benefício apontado por Wikle, Zammit-Mangion et al. (2019) é que a modelagem está baseada no número fixo $r$ de efeitos aleatórios $\boldsymbol{\alpha}$. Nas situações em que $r \ll n$, a performance computacional da inversão de uma matriz $r$-dimensional é muito melhor do que a inversão de uma matriz $n$-dimensional. Para o processo especificado por (3.1), escrevemos $C_{z}=\Phi C_{\alpha} \Phi^{\prime}+C_{\xi}+C_{\epsilon}$ e podemos usar as identidades matriciais de Sherman-Morrison-Woodbury (SEARLE, 1982),

$$
C_{z}^{-1}=\left(C_{\xi}+C_{\epsilon}\right)^{-1}-\left(C_{\xi}+C_{\epsilon}\right)^{-1} \Phi\left(\Phi^{\prime}\left(C_{\xi}+C_{\epsilon}\right)^{-1} \Phi+C_{\alpha}^{-1}\right)^{-1} \Phi^{\prime}\left(C_{\xi}+C_{\epsilon}\right)^{-1}
$$

Se $\left(C_{\xi}+C_{\epsilon}\right)^{-1}$ tem uma estrutura simples (é esparsa ou diagonal) e $r \ll n$, então a inversa é calculada facilmente, por ser uma função de uma matriz simples de alta dimensão e de uma matriz inversa de menor dimensão. Mesmo quando trabalhamos com matriz de ordem completa $(r=n)$ ou super-completa $(r>n)$ pode haver benefícios computacionais devido ao uso de algoritmos multi-resolução, ortogonalidade ou matrizes de precisão esparsas.

A escolha da matriz $\Phi$ é tal que suas colunas são referenciadas no espaço e no tempo. Para processos puramente espaciais ou puramente temporais, costuma-se usar funções de suporte compacto e que possibilitam uma construção multi-resolucional, como uma 
função biquadrática definida em cada localidade fixada $v$ por

$$
\phi(\boldsymbol{u})=\left(1-\left(\frac{\|\boldsymbol{u}-\boldsymbol{v}\|}{R}\right)^{2}\right)^{2} I(\|\boldsymbol{u}-\boldsymbol{v}\|<R),
$$

em que $R$ é um parâmetro de escala e abertura máxima para os valores de $\|\boldsymbol{u}-\boldsymbol{v}\|$. Para o caso espaço-temporal uma possibilidade é considerar o produto tensorial entre duas funções de uma função base espacial $\Phi_{s}$ e uma função base temporal, $\Phi_{t}, \boldsymbol{\Phi}=\boldsymbol{\Phi}_{s} \otimes \boldsymbol{\Phi}_{t}$.

Usa vez escolhidas as funções-base $\Phi$, é possível então obter predição com

$$
\hat{Y}\left(s_{0}, t_{0}\right)=x\left(s_{0}, t_{0}\right)^{\prime} \hat{\boldsymbol{\beta}}+\boldsymbol{\phi}\left(s_{0}, t_{0}\right)^{\prime} C_{\alpha} \Phi C_{z}^{-1}(Z-X \hat{\boldsymbol{\beta}}),
$$

em que

$$
\hat{\boldsymbol{\beta}}=\left(X^{\prime} C_{z}^{-1} X\right)^{-1}\left(X^{\prime} C_{z}^{-1} Z\right),
$$

com o erro padrão da predição da krigagem espaço-temporal universal dado pela raiz quadrada da variância,

$$
\begin{aligned}
\hat{\sigma}_{Y}^{2}\left(s_{0}, t_{0}\right)=\phi & \left(s_{0}, t_{0}\right) C_{\alpha} \phi\left(s_{0}, t_{0}\right)-\phi\left(s_{0}, t_{0}\right) C_{\alpha} \Phi^{\prime} C_{z}^{-1} \Phi C_{\alpha} \phi\left(s_{0}, t_{0}\right) \\
& +\left(x\left(s_{0}, t_{0}\right)-X^{\prime} C_{z}^{-1} \Phi C_{\alpha} \phi\left(s_{0}, t_{0}\right)\right)^{\prime}\left(X^{\prime} C_{z}^{-1} X\right)^{-1}-X^{\prime} C_{z}^{-1} \Phi C_{\alpha} \phi\left(s_{0}, t_{0}\right) .
\end{aligned}
$$

Das Equações (3.14) a (3.18), obtemos que o processo possui custo computacional de $O\left(n r^{2}\right)$. Por ser apenas linear em $n$, o processo de predição se torna viável para grandes mapas quando $r \ll n$.

Estamos agora diante do problema de estimar os parâmetros $\boldsymbol{\theta}=\left\{\boldsymbol{\beta}, \sigma_{\xi}^{2}, \boldsymbol{C}_{\alpha}\right\}$. NGUYEN et al. (2014) argumenta que o uso de bases multi-resolucionais pode resultar em problemas de identificabilidade, especialmente em cenários não-estacionários e nas regiões onde os dados são mais escassos, NychKa, BANDYOPADHYAY et al. (2015) propõe uma estrutura paramétrica para $C_{\alpha}$, em que $C_{\alpha}=C_{\alpha}^{p}(\boldsymbol{\gamma})$, e $\boldsymbol{\theta}=\left\{\boldsymbol{\beta}, \sigma_{\xi}^{2}, \boldsymbol{\gamma}\right\}$.

\subsection{Estimação dos Parâmetros com Algoritmo EM}

Zammit-MAngion e Cressie (2021) propõe a estimação dos parâmetros através do algoritmo de Expectativa-Maximização (EM), baseado na implementação de KATZFUSS e CRESSIE (2011). Seja $L_{c}(\boldsymbol{\theta}) \equiv[\boldsymbol{\alpha}, \boldsymbol{Z} \mid \boldsymbol{\theta}]$ a verossimilhança completa , onde [·] denota a distribuição de probabilidade conjunta de $\alpha$ e $Z$ dado $\theta$. O algoritmo EM primeiramente calcula a esperança condicional (nos dados) da log-verossimilhança dos dados completos para as estimativas atuais dos parâmetros (passo E) e em seguida, maximiza essa função com respeito aos parâmetros (passo $\mathrm{M}$ ). Em notação matemática, o passo E,

$$
Q\left(\boldsymbol{\theta} \mid \boldsymbol{\theta}^{(l)}\right) \equiv E\left(\ln L_{c}(\boldsymbol{\theta}) \mid Z, \boldsymbol{\theta}^{(l)}\right)
$$


é calculado para alguma estimativa atual $\boldsymbol{\theta}^{(l)}$, obtendo a distribuição condicional de $\boldsymbol{\alpha}$ para os valores de $\boldsymbol{\theta}$ estimados no dado momento. $\mathrm{O}$ passo $\mathrm{M}$ atualiza as estimativas dos parâmetros com

$$
\boldsymbol{\theta}^{(l+1)}=\max _{\theta} Q\left(\theta \mid \theta^{(l)}\right)
$$

Usando resultados conhecidos da distribuição condicional Gaussiana (LAIRD e WARE, 1982), encontramos que

$$
\boldsymbol{\alpha} \mid Z, \boldsymbol{\theta}^{(l)} \sim N\left(\boldsymbol{\mu}_{\alpha}^{(l)}, \Sigma_{\alpha}^{(l)}\right)
$$

em que

$$
\begin{gathered}
\boldsymbol{\mu}_{\alpha}^{(l)}=\Sigma_{\alpha}^{(l)} \Phi_{z}^{\prime}\left(C_{\xi}^{(l)}+C_{\epsilon}\right)^{-1}\left(Z-X \boldsymbol{\beta}^{(l)}\right), \\
\Sigma_{\alpha}^{(l)}=\left(\Phi^{\prime}\left(C_{\xi}^{(l)}+C_{\epsilon}\right)^{-1} \Phi+\left(C_{\alpha}^{(l)}\right)^{-1}\right)^{-1}, \\
\boldsymbol{\beta}^{(l+1)}=\left(X^{\prime}\left(C_{\xi}^{(l+1)}+C_{\epsilon}\right)^{-1} X\right)^{-1} X^{\prime}\left(C_{\xi}^{(l+1)}+C_{\epsilon}\right)^{-1}\left(Z-\Phi \mu_{\alpha}^{(l)}\right) .
\end{gathered}
$$

A atualização de $C_{\alpha}^{(l+1)}$ depende da forma de estimação. Caso sejam usados os métodos padrão para estimar a matriz, temos que

$$
C_{\alpha}^{(l+1)}=\Sigma_{\alpha}^{(l)}+\boldsymbol{\mu}_{\alpha}^{(l)} \boldsymbol{\mu}_{\alpha}^{(l)^{\prime}}
$$

Caso uma estrutura paramétrica seja imposta em $C_{\alpha}$, temos que $C_{\alpha}^{(l+1)}=C_{\alpha}\left(\gamma^{(l+1)}\right)$, com atualização dada por

$$
\boldsymbol{\gamma}^{(l+1)}=\underset{\gamma}{\operatorname{argmax}} \ln \left|\mathcal{C}\left(\boldsymbol{\gamma}^{(l)}\right)^{-1}\right|-\operatorname{tr}\left(\mathcal{C}\left(\boldsymbol{\gamma}^{(l)}\right)^{-1}\left(\Sigma_{\alpha}^{(l)}+\boldsymbol{\mu}_{\alpha}^{(l)} \boldsymbol{\mu}_{\alpha}^{(l)^{\prime}}\right)\right) .
$$

Zammit-MAngion e Cressie (2021) propõe função da família exponencial de função de covariâncias

$$
\mathcal{C}_{k}(\gamma)=\left\{\gamma_{1 k} \exp \left(-\frac{d_{i j k}}{\gamma_{2 k}}\right), i, j=1, \ldots, r_{k}\right\},
$$

em que $d_{i j k}$ é a distância entre os centróides da $i$-ésima e da $j$-ésima funções base na $k$-ésima resolução, $r_{k}$ é o número de bases na $k$-ésima resolução, $k=1, \ldots, n_{\text {res }}$ com $n_{\text {res }}$ sendo o número de resoluções, $\gamma_{1 k}$ a variância marginal na $k$-ésima resolução e $\gamma_{2 k}$ é a distância onde a correlação atinge o valor de $e^{-1}$ na $k$-ésima resolução. Então $\mathcal{C}_{k}(\boldsymbol{\gamma})$ é construída como uma matriz bloco-diagonal, com $n_{\text {res }}$ blocos.

Uma vez que $C_{\xi}=\sigma_{\xi}^{2} V_{\xi}, \quad \sigma_{\xi}^{2}$ é atualizada encontrando numericamente os zeros da função traço 


$$
\operatorname{tr}\left(\left(\boldsymbol{C}_{\epsilon}+\left(\sigma_{\xi}^{2}\right)^{(l+1)} \boldsymbol{V}_{\xi}\right)^{-1} \boldsymbol{V}_{\xi}\right)=\operatorname{tr}\left(\left(\boldsymbol{C}_{\epsilon}+\left(\sigma_{\xi}^{2}\right)^{(l+1)} \boldsymbol{V}_{\xi}\right)^{-1} \boldsymbol{V}_{\xi}\left(\boldsymbol{C}_{\epsilon}+\left(\sigma_{\xi}^{2}\right)^{(l+1)} \boldsymbol{V}_{\xi}\right)^{-1} \boldsymbol{\Omega}\right)
$$

em que

$$
\Omega \equiv \boldsymbol{\Phi} \Sigma_{\alpha}^{(l)} \Phi^{\prime}+\Phi \boldsymbol{\mu}_{\alpha}^{(l)} \boldsymbol{\mu}_{\alpha}^{(l)^{\prime}} \Phi^{\prime}-2 \Phi \boldsymbol{\mu}_{\alpha}^{(l)}\left(Z-X \boldsymbol{\beta}^{(l+1)^{\prime}}\right)+\left(Z-X \boldsymbol{\beta}^{(l+1)}\right)\left(Z-X \boldsymbol{\beta}^{(l+1)}\right)^{\prime}
$$

A convergência do algoritmo EM é assegurada usando a log-verossimilhança de dados incompletos em cada iteração

$$
\ln \left[Z \mid \boldsymbol{\beta}^{(l)}, C_{\alpha}^{(l)},\left(\sigma_{\xi}^{2}\right)^{(l)}\right]=-\frac{n}{2} \ln (2 \pi)-\frac{1}{2} \ln \left(\left|C_{z}^{(l)}\right|\right)-\frac{1}{2}\left(Z-X \boldsymbol{\beta}^{(l)}\right)^{\prime}\left(C_{z}^{(l)}\right)^{-1}\left(Z-X \boldsymbol{\beta}^{(l)}\right),
$$

em que

$$
C_{z}^{(l)}=\Phi C_{\alpha}^{(l)}\left(C_{\xi}^{(l)}+C_{\epsilon}\right)
$$

Henderson e SEARle (1981) mostram que as operações matriciais para $C_{z}^{(l)}$ em (3.27) podem ser expressadas por

$$
\begin{aligned}
\left(C_{z}^{(l)}\right)^{-1}= & \left(C_{\xi}^{(l)}+C_{\epsilon}\right)^{-1}- \\
& \left(C_{\xi}^{(l)}+C_{\epsilon}\right)^{-1} \Phi_{Z}\left[\left(C_{\alpha}^{(l)}\right)^{-1}+\Phi_{Z}^{\prime}\left(C_{\xi}^{(l)}+C_{\epsilon}\right)^{-1} \Phi_{Z}\right]^{-1} \Phi_{Z}^{\prime}\left(C_{\xi}^{(l)}+C_{\epsilon}\right)^{-1}, \\
& \left|C_{z}^{(l)}\right|=\left|\left(C_{\alpha}^{(l)}\right)^{-1}+\Phi_{Z}^{\prime}\left(C_{\xi}+C_{\epsilon}\right)^{-1} \Phi_{Z}\right|\left|C_{\alpha}^{(l)}\right|\left|C_{\xi}^{(l)}+C_{\epsilon}\right|
\end{aligned}
$$

e também possuem custo computacional reduzido quando $r<n$ e as funções-base $\phi$ induzem matrizes esparsas. 


\section{Capítulo 4}

\section{Modelo Hierárquico Baseado em Campos Aleatórios}

Uma diferente abordagem considera que um modelo autorregressivo de primeira ordem pode ser imposto para modelar a dependência entre valores passados e procede usando uma representação do Campo Gaussiano como uma combinação linear de funções bases definidas numa triangulação do domínio $\mathcal{D}$. Em casos em que o domínio espacial está disposto em grade regular, o problema aproxima uma modelagem autorregressiva condicional no espaço.

Este capítulo está baseado princialmente no trabalho de LiNDGREN, Håvard RuE et al. (2011) e Håvard Rue, Martino et al. (2009) com a função de covariância de Matérn e com equações estocásticas parciais diferenciais, que expandiram para modelos espaciais as técnicas de aproximação delineadas em Håvard Rue e Held (2005). A modelagem é baseada nos trabalhos de Blangiardo e CAMEletti (2015) e CAMELEtti et al. (2013) e no texto de Blangiardo, Cameletti et al. (2013).

\subsection{Modelo Espaço-Temporal Hierárquico}

Para esse modelo, consideramos o conjunto de observações de um processo $Z$ associadas a um processo latente espaço-temporal $\left\{\eta(s, t): s \in \mathcal{D} \subset \mathbb{R}^{2}, t \in\{1,2 \ldots, T\}\right.$, com $|\mathcal{D}|=m$ e $n=m \times T$.

Considerando um modelo dado por

$$
z(s, t)=x(s, t)^{\prime} \boldsymbol{\beta}+\eta(s, t)+\epsilon(s, t),
$$

com $x(s, t)$ vetor de covariáveis associadas ao processo na localidade $s$ e tempo $t, \boldsymbol{\beta}$ vetor de parâmetros, $\epsilon(s, t) \sim N\left(0, \sigma_{\epsilon}^{2}\right)$ um ruído branco que define o erro de medição. BlAngiaRdo e CAMELETTi (2015) associam o erro $\sigma_{\epsilon}^{2}$ ao chamado efeito Nugget da literatura de geoestatística. 
Em (4.1), $\eta(s, t)$ é a realização do chamado processo de estado, isto é, o real valor não observado do processo, e será assumido como um processo espaço-temporal Gaussiano com evolução no tempo determinada por uma dinâmica autorregressiva e estacionária dada por

$$
\eta(s, t)=a \eta(s, t-1)+\omega(s, t),
$$

para $t=2, \ldots, T, \operatorname{com}|a|<1$ e $\eta(s, 1) \sim N\left(0, \frac{\sigma_{\omega}^{2}}{(1-a)^{2}}\right)$. Ademais, $\omega(s, t)$ é assumida como temporalmente independente e caracterizada pela função de covariância espaço-temporal

$$
\operatorname{Cov}\left(\omega\left(s_{i}, t_{j}\right), \omega\left(\boldsymbol{s}^{\prime}, t^{\prime}\right)\right)=\left\{\begin{array}{ll}
0 & \text { se } t \neq t^{\prime} \\
\sigma_{\omega}^{2} \mathcal{C}(\boldsymbol{h}), & \text { se } t=t^{\prime}
\end{array},\right.
$$

$\operatorname{com} \boldsymbol{h}=\left\|\boldsymbol{s}_{i}-\boldsymbol{s}^{\prime}\right\|$

Escrevemos (4.1) e (4.2) como

$$
\begin{gathered}
Z_{t}=\boldsymbol{X}_{t} \boldsymbol{\beta}+\boldsymbol{\eta}_{t}+\boldsymbol{\epsilon}_{t}, \quad \boldsymbol{\epsilon}_{t} \sim N\left(0, \sigma_{\epsilon}^{2} \boldsymbol{I}\right), \\
\boldsymbol{\eta}_{t}=a \boldsymbol{\eta}_{t-1}+\boldsymbol{\omega}_{t}, \quad \boldsymbol{\omega}_{t} \sim N\left(0, \boldsymbol{Q}_{s}^{-1}\right),
\end{gathered}
$$

sendo $Q_{S}^{-1}$ a matriz de precisão inversa da matriz $\Sigma=\sigma_{\omega}^{2} \Sigma_{s} \operatorname{com}$ elementos $\Sigma_{i, j}=\mathcal{C}\left(\left\|\boldsymbol{s}_{i}-\boldsymbol{s}_{j}\right\|\right)$

O processo latente $\boldsymbol{\eta}=\left\{\boldsymbol{\eta}_{1}^{\prime}, \ldots, \boldsymbol{\eta}_{T}^{\prime}\right\}$ é um campo aleatório Gaussiano de Markov com $\boldsymbol{\eta}_{1} \sim N\left(0, \frac{\sigma_{\omega}^{2} \Sigma_{s}}{\left.1-a^{2}\right)}\right)$, e considerando a hipótese de independência temporal de $\omega(s, t)$, possui distribuição

$$
\boldsymbol{\eta} \sim N\left(\mathbf{0}, Q_{T}^{-1} \otimes Q_{S}^{-1}\right) .
$$

Håvard RuE e Held (2005) mostra que o processo temporal autorregressivo de ordem 1 possui a matriz de precisão

$$
Q_{T}=\left(\begin{array}{ccccc}
\sigma_{\omega}^{2} & -\frac{a}{\sigma_{\omega}^{2}} & & & \\
-\frac{a}{\sigma_{\omega}^{2}} & \frac{\left(1+a^{2}\right)}{\sigma_{\omega}^{2}} & & & \\
& & \ldots & & \\
& & & & \\
& & \frac{\left(1+a^{2}\right)}{\sigma_{\omega}^{2}} & -\frac{a}{\sigma_{\omega}^{2}} \\
& & & -\frac{a}{\sigma_{\omega}^{2}} & \sigma_{\omega}^{2}
\end{array}\right) .
$$

Como discutido na Definição (2.6) e no Teorema 2.1, é conveniente trabalhar com a matriz de precisão quando lidamos com Campos Aleatórios gaussianos de Markov, uma vez que a estrutura de vizinhanças do campo induz a uma matriz esparsa quando 
muitas localidades são condicionalmente independentes duas a duas, dadas as demais. Em particular para o caso espaço-temporal, o custo computacional cai de $O\left(n^{3}\right)$ para $O\left(n^{2}\right)$ nas operações matriciais quando a matriz é esparsa (CAMELETti et al., 2013).

\subsection{Obtenção da Matriz de Precisão Espacial}

A matriz de precisão $Q_{s}$ pode ser obtida por um método em que o processo espacial é considerado um campo Gaussiano com função de covariância $\mathcal{C}(\boldsymbol{h})$ modelada através de uma família de funções de covariância que possuem um papel central em estatística espacial chamada de Função de Covariância de Matérn, e uma representação como campo Gaussiano de Markov é obtida como resultado da solução de uma equação diferencial parcial estocástica. Esse procedimento usa uma partição do domínio espacial $\mathcal{D}$ em triângulos disjuntos que podem ser obtidos através da triangulação de Delaunay (LindgREN, Håvard RuE et al., 2011).

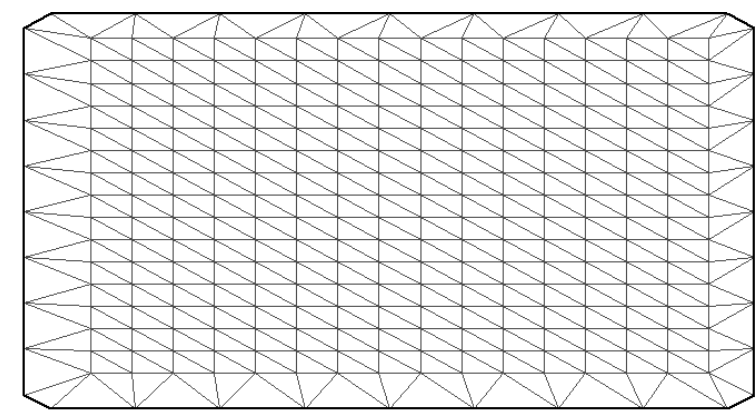

Figura 4.1: Exemplo de Triangulação, usada na simulação desse trabalho. Um vértice de triângulo para cada quatro vértices de área (Triangulação 2), com triângulos a mais nas bordas para as fronteiras.

Considere a seguinte equação diferencial parcial estocástica

$$
\left(\kappa^{2}-\Delta\right)^{\alpha / 2}(\tau Y(s))=W(s), \quad s \in \mathbb{R}^{d},
$$

onde $\Delta=\sum_{i=1}^{n_{s}} \frac{\partial^{2}}{\partial s_{i}^{2}}$ é o Laplaciano, $\tau>0$ controla a variância do processo, $\alpha$ é um parâmetro de suavização, $\kappa$ é um parâmetro de escala e $W(s)$ é ruído branco Gaussiano (ZAMMITMangion e Cressie, 2021; Håvard Rue e Held, 2005). Whittle (1954) e Whittle (1963) usou a definição da transformada de Fourier do Laplaciano fracionário em $\mathcal{R}^{d}$,

$$
\mathcal{F}\left(\left(\kappa^{2}-\Delta\right)^{\alpha / 2} f\right)(\boldsymbol{k})=\left(\kappa^{2}+\|\boldsymbol{k}\|^{2}\right)^{\alpha / 2}(\mathcal{F} f)(\boldsymbol{k})
$$

para mostrar que uma solução da equação diferencial parcial estocástica acima possui função de covariância de Matérn, dada por

$$
C\left(\boldsymbol{s}_{i}, \boldsymbol{s}_{j}\right)=\frac{\sigma^{2}}{2^{v-1} \Gamma(v)}\left(\kappa\left\|\boldsymbol{s}_{i}-\boldsymbol{s}_{j}\right\|\right)^{v} K_{v}\left(\kappa\left\|\boldsymbol{s}_{i}-\boldsymbol{s}_{j}\right\|\right),
$$

com $K_{v}$ a função modificada de Bessel de segundo tipo e ordem $v>0$ Essa função de 
covariância aparece naturalmente em vários problemas práticos (SAMPSON e GUTTORP, 1992). Lindgren, Håvard Rue et al. (2011) estabelece uma relação entre os parâmetros em termos práticos no contexto de campos Gaussianos:

- $\sigma^{2}=\frac{\Gamma(v)}{\Gamma(v+d / 2)(4 \pi)^{(d / 2)} \kappa^{2 v} \tau^{2}}$ é a variância do modelo;

- $\kappa$ está relacionado à distância $l$ em que as correlações atingem o valor empiricamente determinado como 0,13 pela relação $l=\sqrt{8 v} / \kappa$;

- O parâmetro $v$ é frequentemente fixado como 1 ou definido com validação cruzada por ser difícil de interpretar e relacionar com o fenômeno natural e se relaciona com o suavizador da equação diferencial por $\alpha=v+d / 2$, sendo d a dimensão espacial. Nesse trabalho fixamos $v$ como 1.

Para construir uma representação em campo aleatório Gaussiano de Markov para o campo gaussiano com a função de covariância de Matérn, definimos o produto interno

$$
\langle f, g\rangle=\int f(\boldsymbol{u}) g(\boldsymbol{u}) d \boldsymbol{u}
$$

e encontramos uma solução fracamente estocástica (LindgREn, Håvard RuE et al., 2011) para a equação diferencial parcial estocástica que requer

$$
\left\langle\psi,\left(\kappa^{2}-\Delta\right) x\right\rangle \stackrel{D}{=}\langle\psi, W\rangle
$$

para um conjunto finito de funções-base lineares em trecho $\left\{\varphi_{i}(\boldsymbol{u}), \mathrm{i}=1, \ldots \mathrm{k}\right\}, \operatorname{com} \stackrel{D}{=}$ denotando igualdade em distribuição. Tais funções-base são usadas para representar, através da triangularização, a solução da equação diferencial parcial estocástica como

$$
y(\boldsymbol{u})=\sum_{i=1}^{k} \varphi_{i}(\boldsymbol{u}) w_{i},
$$

com $k$ sendo o total de vértices, $\varphi_{i}(\boldsymbol{u})$ as funções que mapeiam cada vértice $k$ nos locais $\boldsymbol{u}$ e determina os pesos $w_{k}$, de distribuição gaussiana, como os valores do campo nos vértices. Os valores no interior do triângulo são determinados por interpolação linear.

Obtemos o sistema de equações lineares

$$
\begin{aligned}
\left\langle\varphi_{j},\left(\kappa^{2}-\Delta\right)\left(\sum_{i} \omega_{i} \varphi_{i}\right)\right\rangle & \stackrel{D}{=}\left\langle\varphi_{j}, W\right\rangle, \quad j=1, \ldots, m . \\
& \left.\left.=\sum_{i}\left(\kappa^{2}\left\langle\varphi_{j}, \varphi_{i}\right\rangle+\right\rangle \nabla \varphi_{j}, \nabla \varphi_{i}\right\rangle\right) \omega_{i}
\end{aligned}
$$

Usando que as integrais estocásticas do ruído branco $W$ são

$$
\int \varphi(s) d W(\boldsymbol{s}) \sim N\left(0, \int \varphi^{2}(\boldsymbol{s}) d \boldsymbol{s}\right)
$$


$\mathrm{e}$

$$
C\left(\int \varphi_{i}(\boldsymbol{s}) d W(\boldsymbol{s}), \int \varphi_{j}(\boldsymbol{s}) d W(\boldsymbol{s})\right)=\int \varphi_{i}(\boldsymbol{s}) \varphi_{j}(\boldsymbol{s}) d \boldsymbol{s},
$$

então o lado direito da Equação (4.13) tem distribuição $N(0, C)$, onde $C_{i j}=\int \varphi_{i}(s) \varphi_{j}(s) d s$ (Bolin, 2015).

Usando a primeira identidade estocástica de Green (Lindgren, Håvard Rue et al., 2011), escrevemos lado esquerdo de (4.13) como

$$
\begin{aligned}
\left\langle\varphi_{j},\left(\kappa^{2}-\Delta\right)\left(\sum_{i} w_{i} \varphi_{i}\right)\right\rangle & =\sum_{i}\left\langle\varphi_{j},\left(\kappa^{2}-\Delta\right) w_{i} \varphi_{i}\right\rangle \\
& =\sum_{i}\left(\kappa^{2}\left\langle\varphi_{j}, \varphi_{i}\right\rangle+\left\langle\nabla \varphi_{j}, \nabla \varphi_{i}\right\rangle\right) w_{i} .
\end{aligned}
$$

Partindo de duas matrizes:

$$
\begin{aligned}
C_{i i} & =\int \varphi_{i}(s) d s \quad \text { (os termos constantes) } \\
G_{i j} & =\int \nabla \varphi_{i}(s) \cdot \nabla \varphi_{j}(s) d s \quad \text { (Os termos do Laplaciano) }
\end{aligned}
$$

a equação diferencial parcial estocástica em (4.13) se torna

$$
\left(\kappa^{2} C+G\right) \omega \sim N(0, C),
$$

e, dessa forma, $\omega$ é um campo Gaussiano de Markov com matriz de precisão (Bolin, 2015)

$$
Q_{s}=\left(\kappa^{2} C+G\right)^{\prime} C^{-1}\left(\kappa^{2} C+G\right) .
$$

Nota-se que a matriz $C^{-1}$ não é esparsa. Lindgren, Håvard Rue et al. (2011) mostra com resultados clássicos da teoria de Métodos de Elementos Finitos que $C$ pode ser substituída por $\tilde{\boldsymbol{C}}=\tilde{\boldsymbol{C}}_{i i}=\left\langle\varphi_{i}, 1\right\rangle$. O custo computacional resulta em $O(n)$ para qualquer triangulação.

LINDGREN, Håvard RUE et al. (2011) também descreve algumas equações parciais diferenciais estocásticas que permitem generalizações e ainda têm soluções em campos de Matérn, como para campos não estacionários, campos que apresentam características oscilatórias, modelos de equações parciais diferencias estocásticas não separáveis no espaço-tempo e modelos não-isotrópicos.

No caso em que as localidades dos dados estão dispostas em uma grade regular bidimensional (como no caso em que as áreas medidas correspondem aos pixels de uma imagem), Lindgren, Håvard Rue et al. (2011) mostra como a triangulação em (4.12) se resume ao caso autorregressivo condicional (CAR) definido em BESAG (1974).

Considerando $y$ como um campo Gaussiano de Markov com as esperanças condicionais dadas por 


$$
E\left(y_{i j} \mid y_{-i j}\right)=\frac{1}{c}\left[d\left(y_{i-1, j}+y_{i+1, j}\right)+b\left(y_{i, j-1}+y_{i, j+1}\right)\right],
$$

e variâncias condicionais dadas por

$$
\operatorname{Var}\left(y_{i j} \mid \boldsymbol{y}_{-i j}\right)=\frac{1}{c}
$$

sendo $b, d$ pesos associados às direções horizontal e vertical no cálculo da esperança condicional. BESAG (1981) mostra que a covariância entre dois pontos na grade pode ser aproximada por

$$
C\left(y_{i j}, y_{i^{\prime} j^{\prime}}\right) \approx \frac{1}{2 \pi} K_{0}\left\|y_{i j}-y_{i^{\prime} j^{\prime}}\right\| \sqrt{c-2 d-2 b},
$$

resultado que pode ser obtido tomando a Equação (4.10) no limite $v \rightarrow 0$, com $\kappa^{2}=$ $c-2 d-2 b$ e $\sigma^{2}=\frac{1}{4 \pi}$. Isso significa que o modelo autorregressivo condicional gera soluções aproximadas para a Equação (4.8) na grade, $\operatorname{com} v=0$.

\subsection{Estimação dos Parâmetros}

Os parâmetros do modelo proposto em (4.5) podem ser estimados usando o algoritmo de integração aproximada aninhada de Laplace (Integrated Nested Laplace Approximation, ou INLA). Essa abordagem, proposta em Håvard Rue, MARTino et al. (2009), foi desenvolvida como uma alternativa aos métodos Markov Chain Monte Carlo para obtenção de marginais a posteriori em inferência Bayesiana e apresenta grandes vantagens computacionais (BolIn, 2015) ao substituir o processo de reamostragem do MCMC por uma integração numérica.

Consideramos o conjunto de parâmetros a estimar $\boldsymbol{\theta}=\left(\sigma_{\omega}^{2}, \sigma_{\epsilon}^{2}, \kappa, a\right)$ e denotando como $l=\{\boldsymbol{\eta}, \boldsymbol{\beta}\}$ conjunto incluindo o campo gaussiano latente assumindo $\boldsymbol{\eta}$ e $\boldsymbol{\beta}$ independentes a priori. Atribuímos uma priori Gaussiana vaga com matriz de covariâncias conhecida a $\boldsymbol{\beta}$ e a distribuição do campo Gaussiano de Markov com a matriz de covariâncias $\Sigma$ a $\boldsymbol{\eta}$. Assim, $\boldsymbol{\eta}, \boldsymbol{\beta} \mid \boldsymbol{\theta} \sim N\left(\mathbf{0}, \Sigma\left(\sigma_{\omega}^{2}, a, \kappa\right)\right)$ e $\boldsymbol{Z}_{t} \mid \boldsymbol{\eta}, \boldsymbol{\beta}, \boldsymbol{\theta} \sim N\left(\boldsymbol{X}_{t} \boldsymbol{\beta}+\boldsymbol{\Phi} \boldsymbol{\eta}_{t}, \sigma_{\epsilon}^{2} I\right)$. CAmeletti et al. (2013) mostra que a distribuição conjunta a posteriori é dada por

$$
f(\boldsymbol{l}, \boldsymbol{\theta} \mid \boldsymbol{Z})=f(\boldsymbol{\theta}) f(\boldsymbol{l} \mid \boldsymbol{\theta}) \prod_{t=1}^{T} f\left(\boldsymbol{Z}_{t} \mid \boldsymbol{l}, \boldsymbol{\theta}\right)
$$

As distribuições marginais posteriores são dadas por

$$
\begin{gathered}
f(\boldsymbol{l} \mid Z)=\int f\left(l_{i} \mid \boldsymbol{\theta}, Z\right) f(\boldsymbol{\theta} \mid Z) d \boldsymbol{\theta}, \\
f(\boldsymbol{\theta} \mid Z)=\int f(\boldsymbol{\theta} \mid Z) d \boldsymbol{\theta}_{-j},
\end{gathered}
$$


com $i=1, \ldots, T$ e $j=1, \ldots, 4$. O método MCMC simularia e reamostraria essas distribuições em um processo demorado. Através do algoritmo INLA pode-se obter uma aproximação $\tilde{f}(\theta \mid Z)$ para a distribuição dos parâmetros de $\boldsymbol{\theta}$.

Da definição de probabilidade condicional, obtemos que

$$
f(\boldsymbol{\theta} \mid Z)=\frac{f(\boldsymbol{l}, \boldsymbol{\theta} \mid Z)}{f(\boldsymbol{l} \mid \boldsymbol{\theta}, Z)}
$$

Propõe-se uma aproximação para a densidade conjunta a posteriori de $\theta$ como

$$
\left.\left.f(\boldsymbol{\theta} \mid Z) \propto \frac{f(\boldsymbol{l}, \boldsymbol{\theta}, Z)}{f(\boldsymbol{l} \mid \boldsymbol{\theta}, Z)}\right|_{x=x^{\star}} \approx \frac{f(\boldsymbol{l}, \boldsymbol{\theta}, Z)}{\tilde{f}(\boldsymbol{l} \mid \boldsymbol{\theta}, Z)}\right|_{\boldsymbol{l}=\boldsymbol{l}^{*}},
$$

em que $\tilde{f}(\boldsymbol{l} \mid \boldsymbol{\theta}, Z)$ é a aproximação Gaussiana da condicional de $\boldsymbol{l}$ dada por

$$
f(\boldsymbol{l}, \boldsymbol{\theta}, Z) \propto \exp \left(-\frac{1}{2} \boldsymbol{l}^{\prime} Q(\boldsymbol{\theta}) \boldsymbol{l}+\sum_{i} \log \left(f\left(Z_{i}, l_{i}\right)\right)\right),
$$

e $\boldsymbol{l}^{*}$ é a moda da condicional completa de $\boldsymbol{l}$ dado $\boldsymbol{\theta}$, obtida através de algum método de otimização como Newton-Raphson. Para mais detalhes sobre essa aproximação e a obtenção de $\boldsymbol{l}^{\star}$, ver Håvard RuE, MARTino et al. (2009).

A distribuição de $l \mid Z$ pode ser obtida de forma exata por ser Gaussiana. MARTino e Riebler (2020) mostram que, obtendo-se $f(\boldsymbol{\theta} \mid Z)$ para todos os $k$ pontos definidos pela função-base $\phi_{i}(\boldsymbol{s})$, obtemos a solução da Equação (4.22) numericamente como

$$
f(\boldsymbol{l} \mid Z)=\sum_{i=1}^{K} f\left(\boldsymbol{l} \mid \boldsymbol{\theta}^{k}, \boldsymbol{Z}\right) \tilde{f}\left(\boldsymbol{\theta}^{k} \mid Z\right) \phi_{i k}
$$

e é imediata a predição para $z(s, t)$ para a triangulação mapeada. 



\section{Capítulo 5}

\section{Estudos de Simulação}

No Capítulo 3, foram apresentadas as definições e os conceitos sobre modelos de Krigagem e uma nova abordagem baseada em modelos mistos com redução de dimensionalidade a partir do uso de bases espaço-temporais denominada Krigagem de Posto Fixo (Cressie e Johannesson, 2008). No Capítulo 4, apresentamos uma abordagem baseada em modelos hierárquicos para a predição de observações faltantes, baseada nos modelos espaço-temporais hierárquicos descritos em Håvard RuE, MARTino et al. (2009).

O objetivo deste capítulo é comparar os resultados obtidos para a interpolação de dados faltantes com as abordagens apresentadas nos Capítulos 3 e 4 . Foram realizadas simulações de conjuntos espaço-temporais com características semelhantes aos conjuntos de dados obtidos de imagens de satélite, incluindo os perfis de dados faltantes que são comumente observados nesse tipo de conjunto. Para a predição, foram utilizados modelos de krigagem universal, Krigagem de Posto Fixo, modelos autorregressivos condicionais e modelos espaço-temporais hierárquicos baseados em campos Gaussianos com função de covariância de Matérn.

As simulações foram realizadas em servidor com processador Intel Xeon E5-2650 de 12 núcleos e 384 GB de memória RAM.

\subsection{Descrição dos Processos de Simulação}

Neste estudo de simulação, sejam $t=1,2, \ldots, T$ o tempo, e $s=s_{1}, s_{2}, \ldots, s_{N} \in \mathcal{D}$, as $N$ regiões de uma localidade divididas em grade. Supomos que o processo espacial é fracamente estacionário, isto é,

- $E[Z(s, t)]=\mu \mathrm{e}$

- $\operatorname{Cov}(Z(\boldsymbol{s}+\boldsymbol{h}, t+\tau))=\operatorname{Cov}(Z(\boldsymbol{h}, \tau), Z(\mathbf{0}, 0))=C(\boldsymbol{h}, \tau) \forall t \in T, \quad \forall \boldsymbol{h} \in \mathcal{D} ; \tau \in \mathbb{R}, \operatorname{com}$ $\boldsymbol{s}+\boldsymbol{h} \in \mathcal{D}$ e $t+\tau \in \mathbb{R}$.

As simulações foram realizadas tomando um caso especial da função de covariância separável apresentada em (3.11) chamado modelo de covariância espaço-temporal expo- 
nencial, com parâmetro de variância $\sigma^{2}$, parâmetro de decaimento espacial $v_{s}$ e parâmetro de decaimento temporal $v_{t}$ dado por

$$
C(\boldsymbol{h}, \tau, \boldsymbol{\theta})=\sigma^{2} e^{\left(-v_{s}\|\boldsymbol{h}\|\right)} e^{\left(-v_{t} \tau\right)},
$$

em que $\boldsymbol{\theta}=\left(\sigma^{2}>0, v_{s}>0\right.$ e $\left.v_{t}>0\right)($ Jin, 2017).

Nesta simulação de um campo Gaussiano, temos que

$$
\left[\begin{array}{c}
Z\left(s_{1}, 1\right) \\
\ldots \\
Z_{\left(s_{N}, 1\right)} \\
\ldots \\
Z\left(s_{1}, T\right) \\
\ldots \\
Z\left(s_{N}, T\right)
\end{array}\right] \sim N\left(\left[\begin{array}{c}
\mu \\
\cdots \\
\mu \\
\cdots \\
\mu \\
\cdots \\
\mu
\end{array}\right], \Sigma_{N T \times N T}\right),
$$

em que $\Sigma$ é a matriz de covariância espaço-temporal dada por $C(\boldsymbol{h}, \tau, \boldsymbol{\theta})$.

A simulação do campo Gaussiano foi obtida com o auxílio do pacote RandomFields do software R (Schlather, Malinowski, Menck et al., 2015) (R Core Team, 2020). Dez diferentes conjuntos de dados em grade representando uma área de $N=32 \times 32=1024$ pixels em $T=64$ tempos foram gerados a partir da função de covariância espaço-temporal exponencial com variância $\sigma^{2}=3$ e escalas $v_{s}=v_{t}=5$, de forma que o resultado da simulação seja similar a uma série de dados de temperatura de superfície. Uma outra avaliação é realizada com dez conjuntos gerados a partir das mesmas sementes mas com um efeito sazonal de 16 tempos incluso, a fim de melhor representar um típico conjunto de variáveis relacionadas aos fatores climáticos.

Uma vez obtidos os conjuntos de dados, uma nova simulação é feita a fim de se determinar os pixels que serão removidos. Novamente com o auxílio do pacote RandomFields e uma função de covariância espaço-temporal exponencial, os pixels que serão removidos são escolhidos de forma a representar as diferentes situações em que um satélite de sensoriamento remoto pode não conseguir produzir a informação: presença aleatória de artefatos que afetam o sensor em uma área específica, presença de partículas poluentes na atmosfera (o que ocorre tipicamente em áreas urbanas ou industriais, onde a temperatura de superfície também tende a ser maior) e presença de nuvens (que ocorrem em maior número durante meses específicos do ano). As Figuras 5.1 e 5.2 ilustram um exemplo de simulação e seu aspecto após a remoção de dados. 


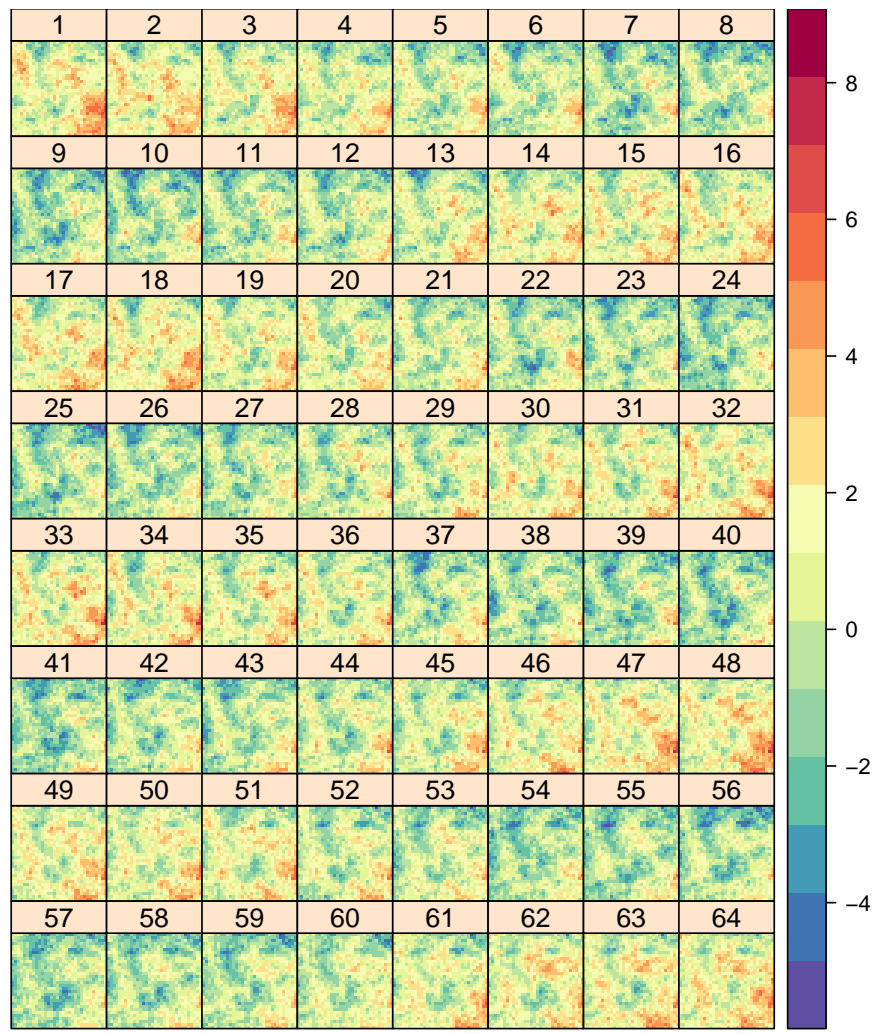

Figura 5.1: Exemplo de simulação de um conjunto com área $32 \times 32$ pixels em 64 tempos e sazonalidade de 16 tempos. Nessa figura, a cor dos pontos representa o valor simulado.

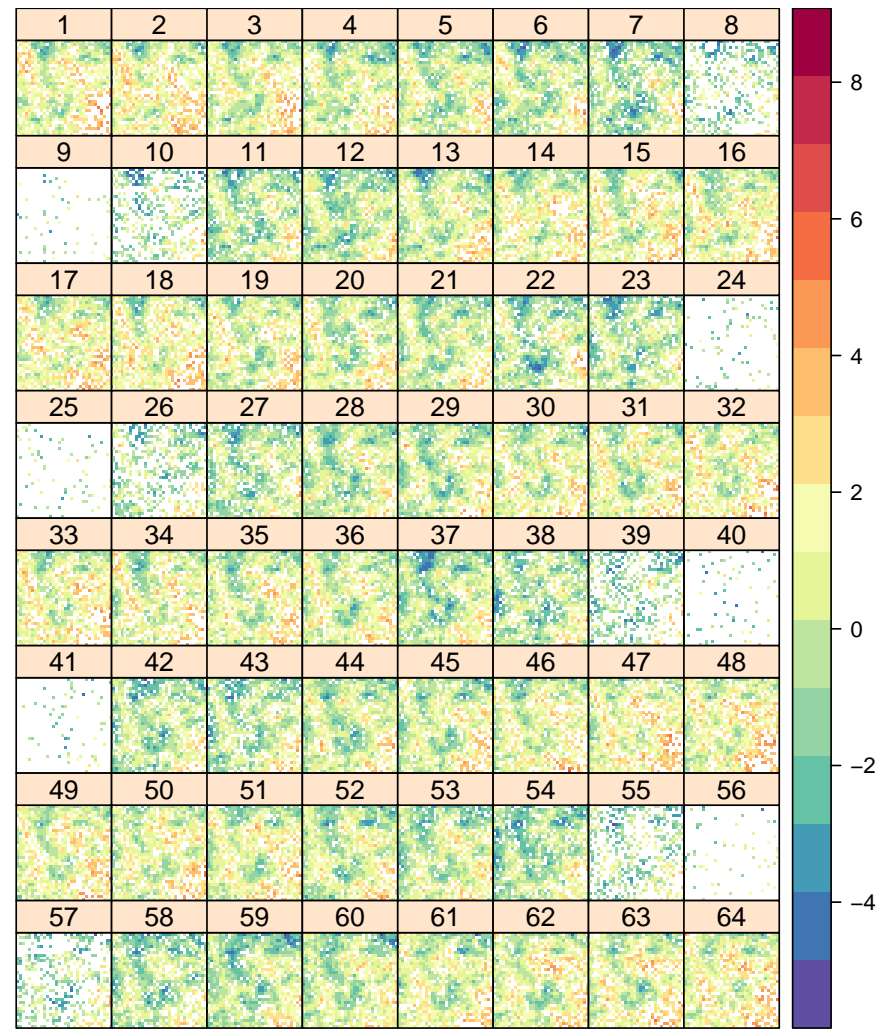

Figura 5.2: A mesma simulação apresentada acima, com dados removidos. 


\subsection{Descrição dos Modelos Avaliados}

Neste capítulo, foram avaliadas quatro abordagens distintas para a interpolação de dados faltantes em um conjunto espaço-temporal:

- Krigagem universal, descrita na Seção 3.1 e com predição descrita pela Equação (3.9). Foi utilizada a função krigeST do pacote gstat (GRÄLER et al., 2016) do software R. Esse método demanda a construção de um semivariograma tal como definido na Seção 2.4. Devido ao grande volume de dados, faz-se necessário delimitar a quantidade de dados a serem utilizados na formulação conjunta apresentada em (3.4), escolhendo um número de áreas vizinhas mais próximas. Isso também implica na necessidade de se definir uma relação de escala entre a variação no espaço e a variação no tempo;

- Krigagem de Posto Fixo - FRK (do inglês Fixed Rank Kriging), apresentada na Seção 3 e dada pela equação (3.12), com predição dada pela Equação (3.16). Para este método foram testados dois conjuntos $\phi$ de funções-base, com duas e três resoluções no espaço e uma no tempo. O pacote FRK do software R apresenta uma implementação recente desse método (ZAMmit-MAngion e Cressie, 2021)

- Modelo Autorregressivo Condicional (CAR), dado pela equação (2.13) (BESAG, 1981). Implementações desse modelo costumam demandar grande volume de recursos computacionais, mas a abordagem baseada no resultado assintótico apresentado na Seção 4.2 e com predição usando o algoritmo de integração aproximada aninhada de Laplace descrito na Seção 4.3 promete grandes vantangens computacionais. O pacote INLA do software R (Håvard Rue, MARTino et al., 2009) implementa esse método e é bastante robusto, permitindo o uso de priori para hiper-parâmetros, dentre outras possibilidades.

- Modelo Espaço-Temporal Hierárquico baseado em campo Gaussiano com função de covariância de Mátern, tal como descrito no Capítulo 4 e representado pelas Equações (4.4) e (4.5). Duas diferentes partições do domínio espacial em triângulos são avaliadas para gerar a matriz de precisão $Q_{s}$. O pacote INLA do software R também implementa o método de solução de equações diferenciais parciais estocásticas utilizados para estimar $Q_{s}$. (LindGREN, Håvard Rue et al., 2011)

As implementações dos modelos disponíveis nos pacotes gstat, FRK e INLA foram escolhidas devido à capacidade implementada nesses pacotes de gerar não apenas as predições como também estimar o erro padrão dessas predições. A função krigeST do pacote gstat não produz predições para os pontos que não são faltantes.

Os modelos descritos acima foram avaliados considerando os seguintes parâmetros:

(a) Krigagem:

- Relação entre variações de espaço e tempo igual a 1;

- Número de vizinhos a se considerar igual a 118; 
- Função de covariância separável exponencial no espaço e no tempo.

(b) FRK 1:

- Número de funções-base temporais $\phi_{t}$ igual a 20;

- Número de funções-base espaciais $\phi_{s}$ igual a 162, gerando, após o produto tensorial, 3240 bases $\phi$;

- Função-base biquadrática, com abertura igual a 1 e regularmente distribuída na grade;

- 5 iterações do algoritmo EM com tolerância da convergência igual a 0,001.

(c) FRK 2:

- Número de funções-base temporais $\phi_{t}$ igual a 20;

- Número de funções-base espaciais $\phi_{s}$ igual a 216, gerando, após o produto tensorial, 4320 bases $\phi$;

- Função-base biquadrática, com abertura igual a 1 e regularmente distribuída na grade;

- 5 iterações do algoritmo EM com tolerância da convergência igual a 0,001.

(d) CAR:

- Estrutura temporal definida segundo modelo autorregressivo de ordem 1, com priori para a autocorrelação $a$ tal que $P(a>0)=0,9$.

(e) Triangulação 1:

- Um vértice de triângulo para cada 1,5 áreas do conjunto de dados.

- Estrutura temporal definida segundo modelo autorregressivo de ordem 1, com priori para a autocorrelação $a$ tal que $P(a>0)=0,9$.

(f) Triangulação 2:

- Um vértice de triângulo para cada 2 áreas do conjunto de dados.

- Estrutura temporal definida segundo modelo autorregressivo de ordem 1, com priori para a autocorrelação $a$ tal que $P(a>0)=0,9$.

Para o conjunto em que foi acrescentado um efeito sazonal na simulação, o ajuste foi realizado considerando o tempo como variável categórica no ajuste do modelo. 


\subsection{Avaliação do Ajuste}

Para cada um dos dez conjuntos simulados e dos seis modelos escolhidos, a avaliação ocorreu através do erro quadrático médio (de predição), definido por

$$
E Q M=\frac{1}{N} \frac{1}{T} \sum_{s \in \mathcal{D}} \sum_{t=1}^{T}(\hat{Z}(s, t)-Z(s, t))^{2}
$$

onde $N=1024$ se refere ao número de locações espaciais $s \in \mathcal{D}$ em cada tempo $t, T=64$ é o número de tempos simulados e $\hat{Z}(s, t)$ valor ajustado pelo modelo para $Z(s, t)$.

Adicionalmente, será avaliado, de maneira descritiva, o viés da predição, dado por

$$
\operatorname{vies}(s, t)=(\hat{Z}(s, t)-Z(s, t))
$$

Por fim, uma validação foi analisada considerando o erro padrão estimado para a predição. Foi calculado o seguinte intervalo

$$
I C(s, t)=[\hat{Z}(s, t)-1,96 e p(\hat{Z}(s, t)) ; \hat{Z}(s, t)+1,96 \operatorname{ep}(Z(\hat{s}, t))],
$$

e avaliado se o valor simulado $Z(s, t)$ está incluso no intervalo.

\subsection{Ilustração dos resultados}

Os resultados obtidos para a simulação ilustrada na Figura 5.2 são apresentados nesta Seção a fim de ilustrar a avaliação que foi realizada. Na Figura 5.3 apresentamos os mapas resultantes da predição dos modelos. As Figuras 5.4 e 5.5 mostram os gráficos de dispersão entre o valor real e o predito, para as observações deixadas no conjunto e para as removidas, respectivamente. Nessas figuras, vemos que o modelo CAR apresenta melhores resultados tanto para os dados mantidos quanto para os removidos, mas se mostra particularmente sensível para a remoção de dados em comparação aos demais modelos, com a Triangulação 1 apresentando desempenho próximo para a predição das observações suprimidas. Uma possível explicação para esse fato é o uso, nas triangulações, da função de covariância de Matérn, enquanto o modelo CAR se vale de um resultado assintótico.

As Figuras 5.6 e 5.7 mostram os gráficos do erro quadrático médio da predição, calculados para cada dia, pelo número de dias faltantes no conjunto. Nessas figuras, vemos que o método FRK apresentou erro quadrático médio maior, sobretudo para as observações removidas, enquanto o método CAR apresentou os melhores resultados.

A Figura 5.8 apresenta os vieses em forma de mapa, onde é possível observar os maiores vieses no método FRK e os menores no método CAR, bem como uma proporção maior de vieses menores que zero sobretudo para o método com as triangulações. A Figura 5.9 apresenta mapa com os erros padrões estimados e a Figura 5.10 aponta se os valores reais ficam dentro dos intervalos calculados pela Equação (5.5). 


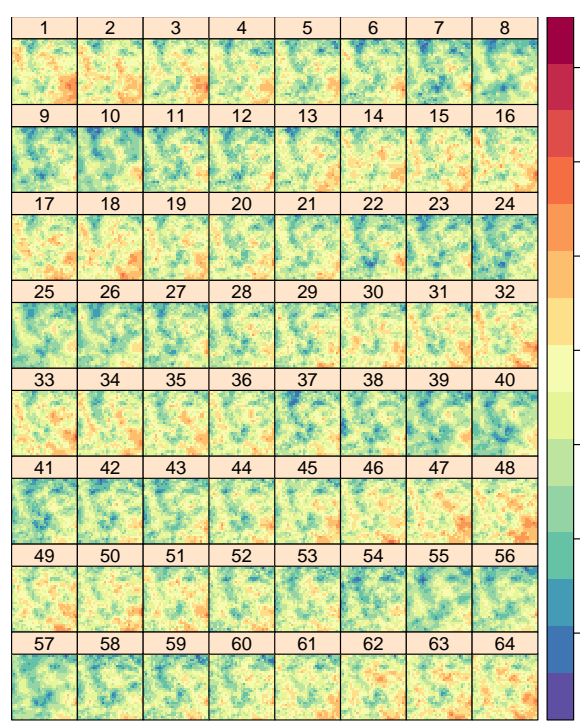

(a) Krigagem

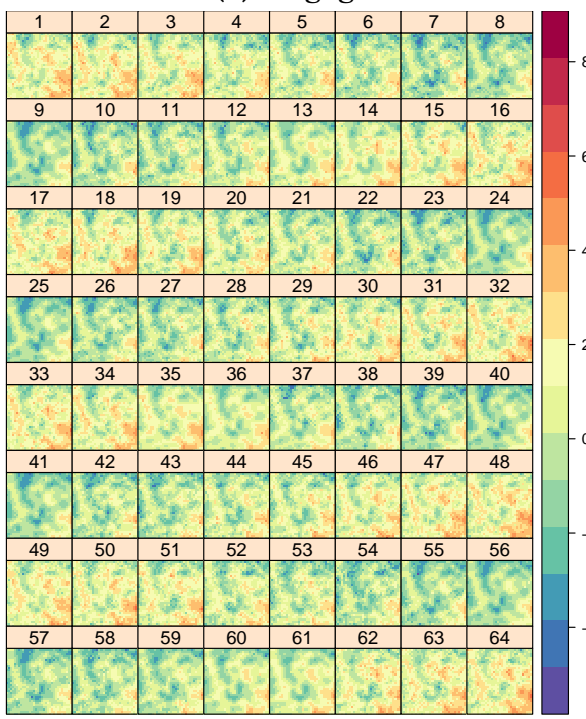

(c) FRK 2

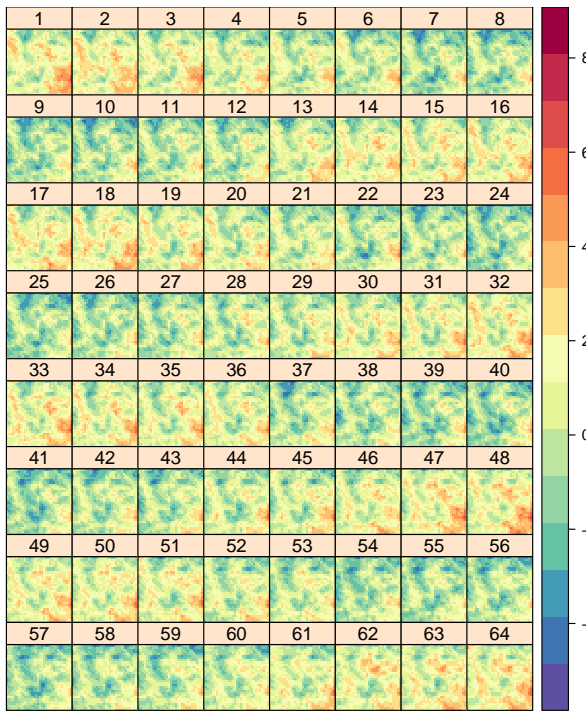

(e) Triangulação 1

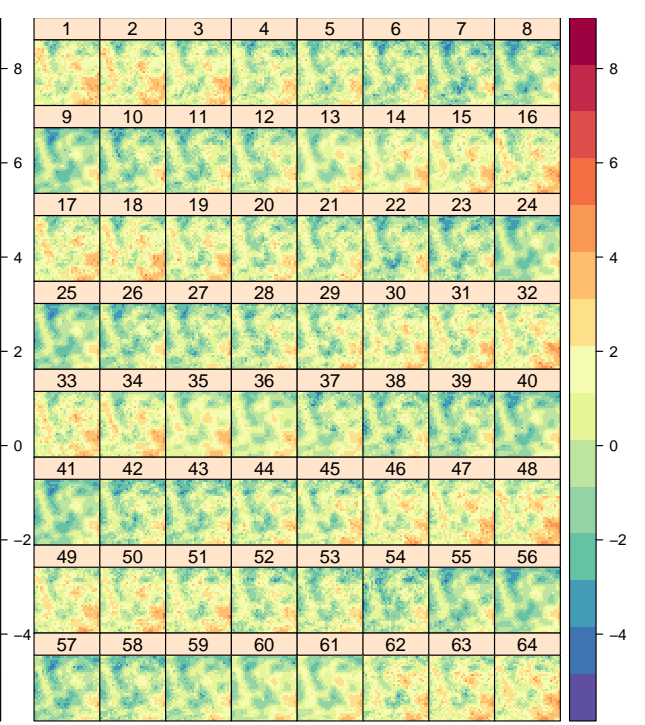

(b) FRK 1

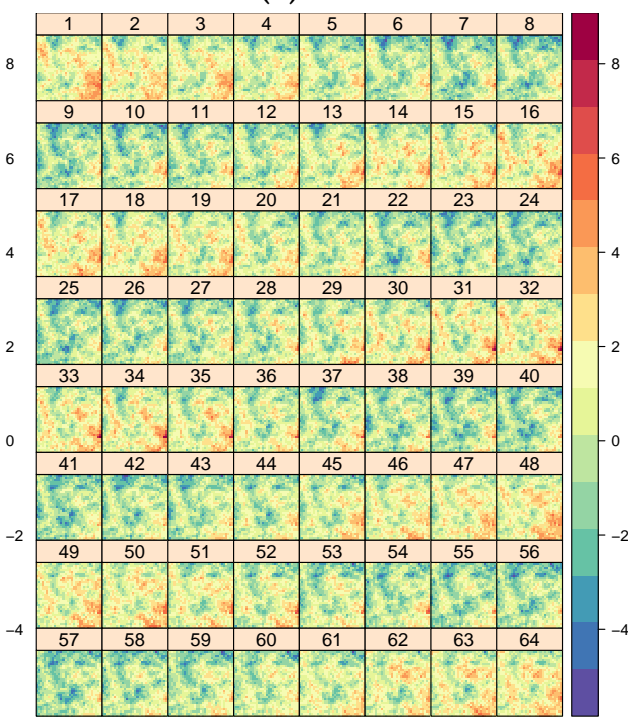

(d) $C A R$

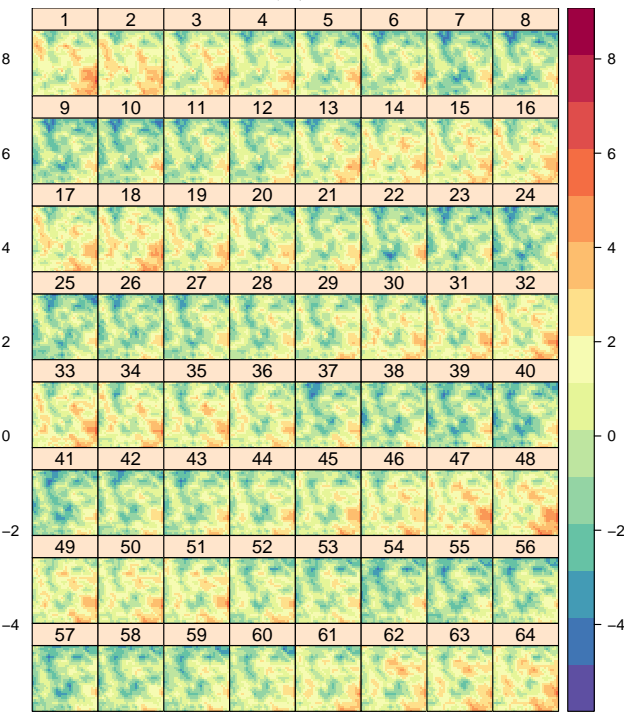

(f) Triangulação 2

Figura 5.3: Predições para a Simulação apresentada na Figura 5.1, com os métodos descritos nessa seção. 


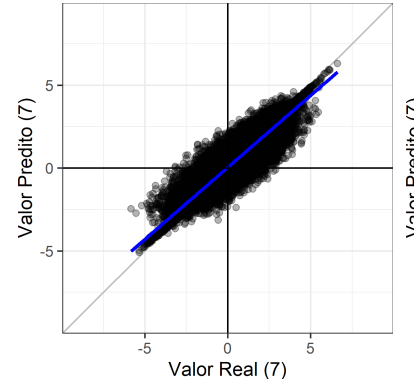

(a) FRK 1

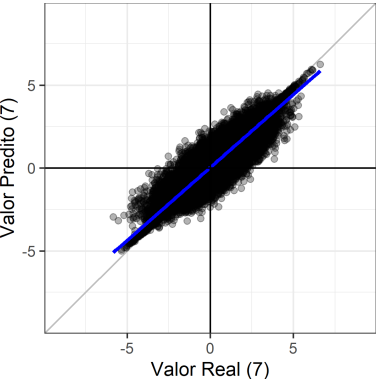

(b) FRK 2

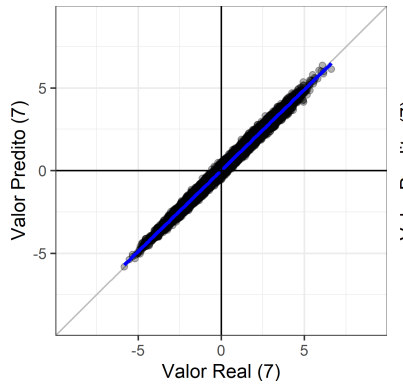

(c) $C A R$

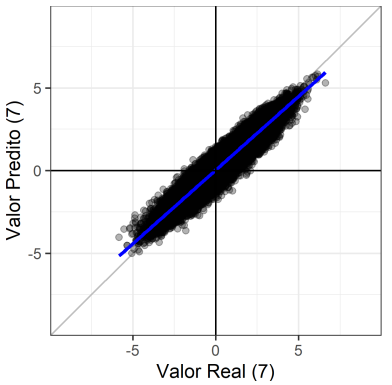

(d) Triangulação 1

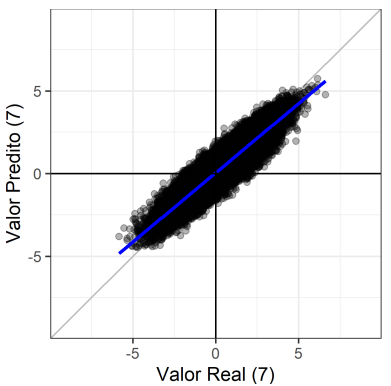

(e) Triangulação 2

Figura 5.4: Gráficos de dispersão entre o valor real e o valor predito para as observações que estão no conjunto.

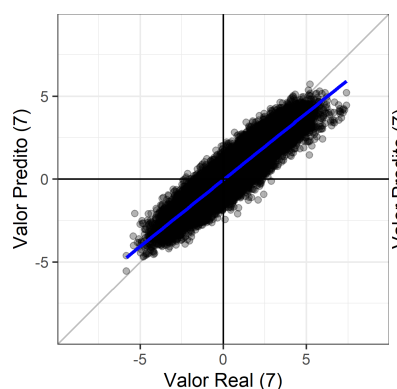

(a) Krigagem

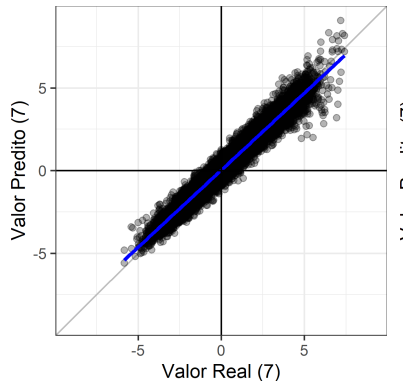

(d) $C A R$

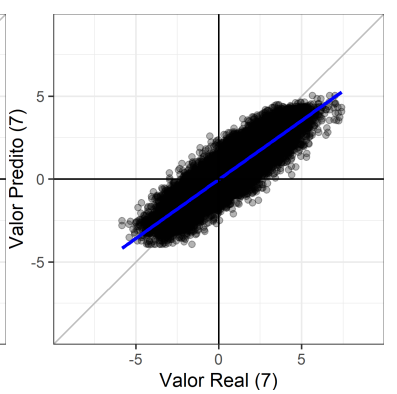

(b) FRK 1

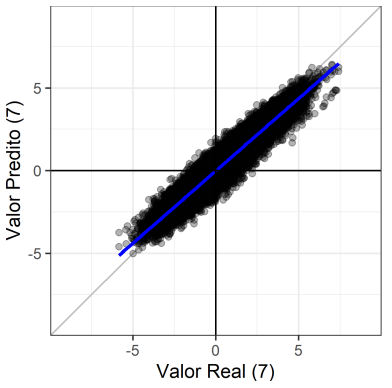

(e) Triangulação 1

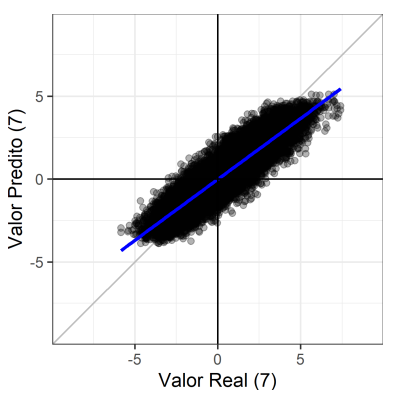

(c) FRK 2

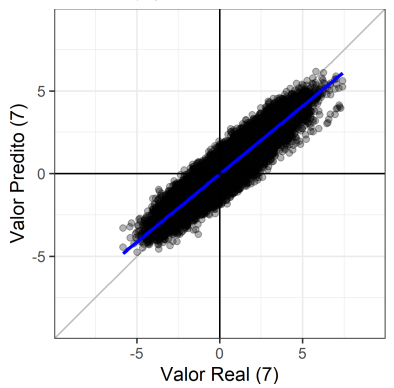

(f) Triangulação 2

Figura 5.5: Gráficos de dispersão entre o valor real e o valor predito para as observações que foram removidas do conjunto simulado. 


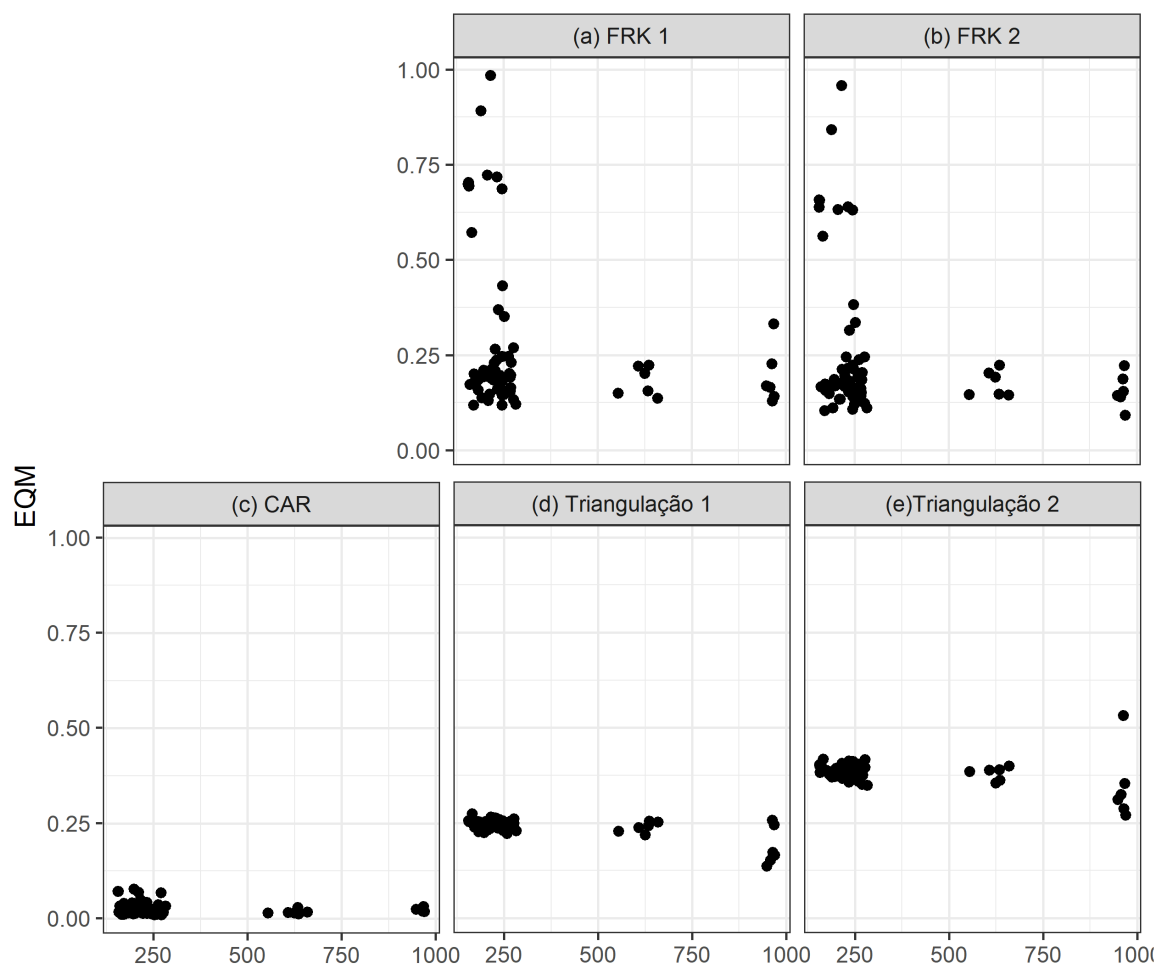

Figura 5.6: Gráficos de dispersão entre o erro quadrático médio entre as observações mantidas no conjunto em cada dia pelo número de observações faltantes no conjunto.

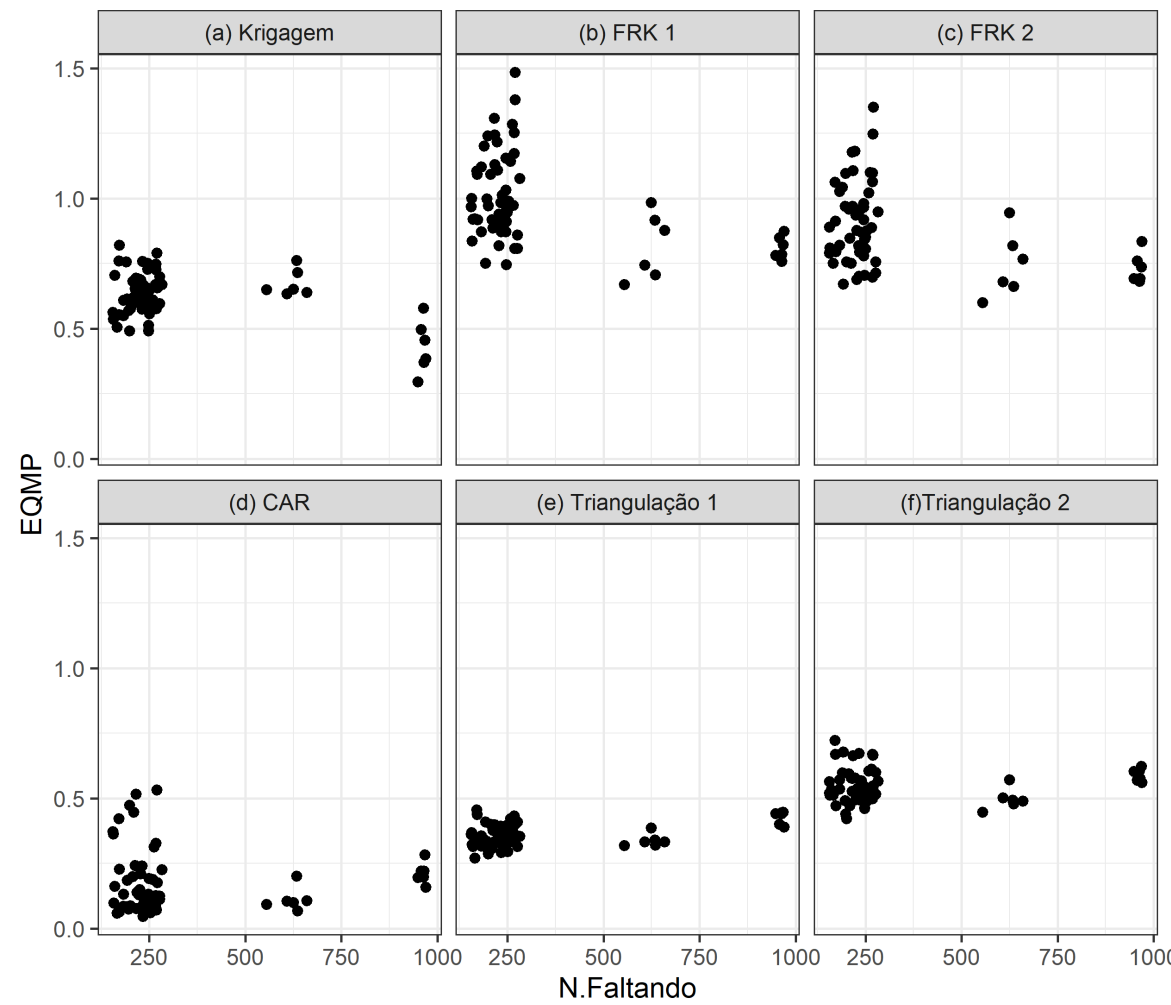

Figura 5.7: Gráficos de dispersão entre o erro quadrático médio de predição entre as observações suprimidas do conjunto em cada dia pelo número de observações faltantes no conjunto. 


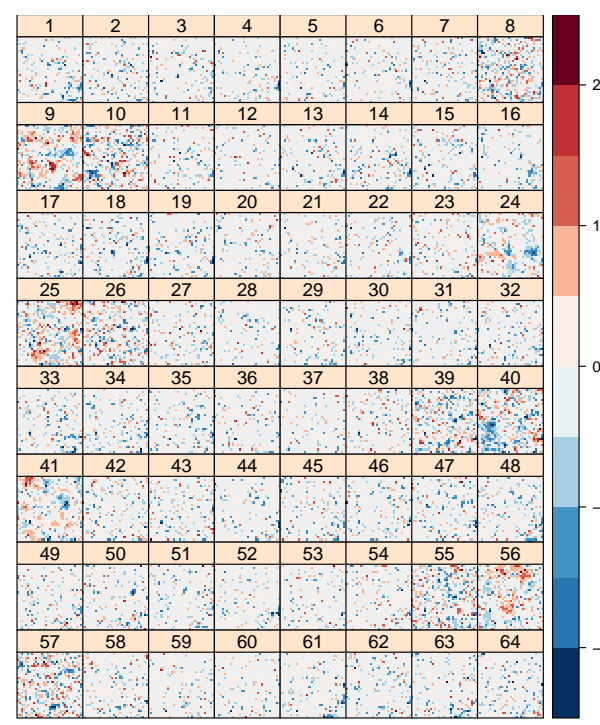

(a) Krigagem

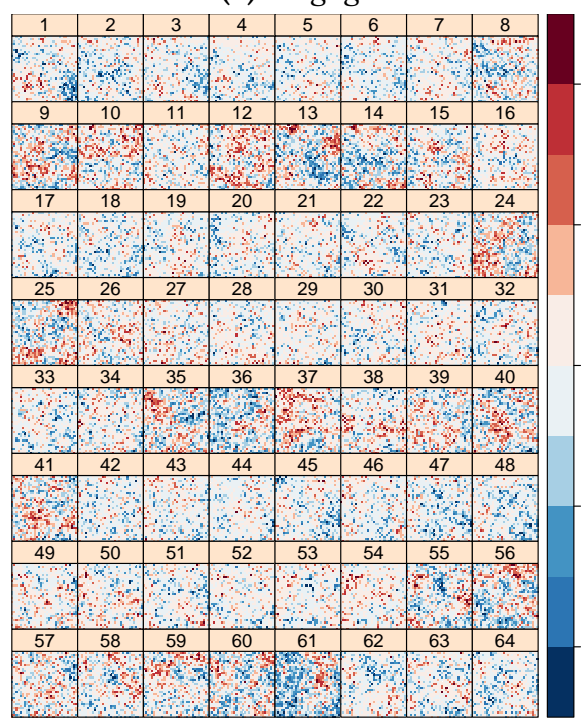

(c) FRK 2

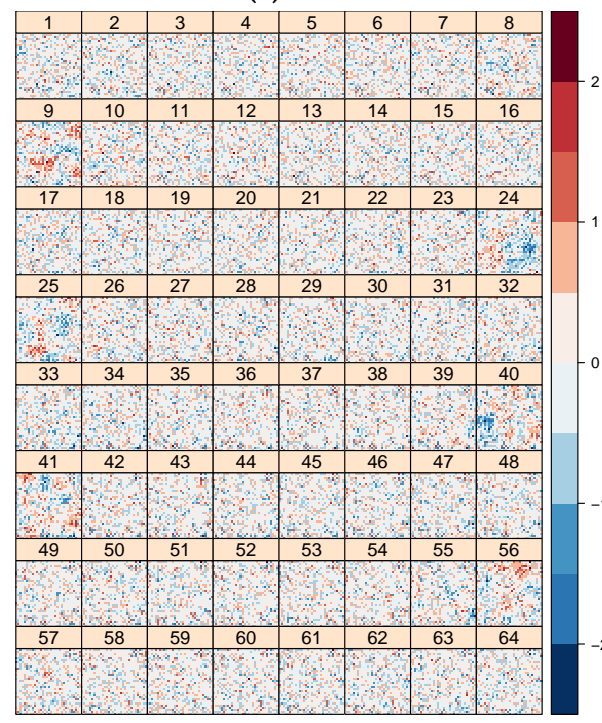

(e) Triangulação 1

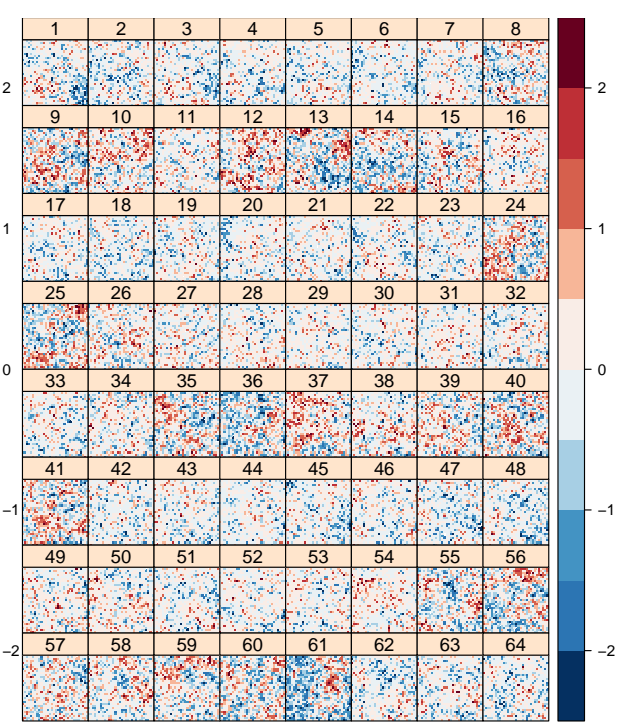

(b) FRK 1

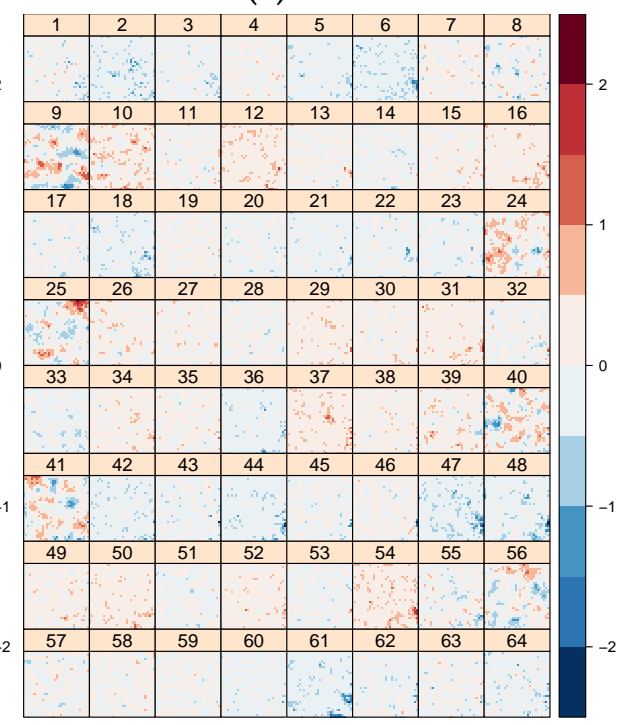

(d) $C A R$

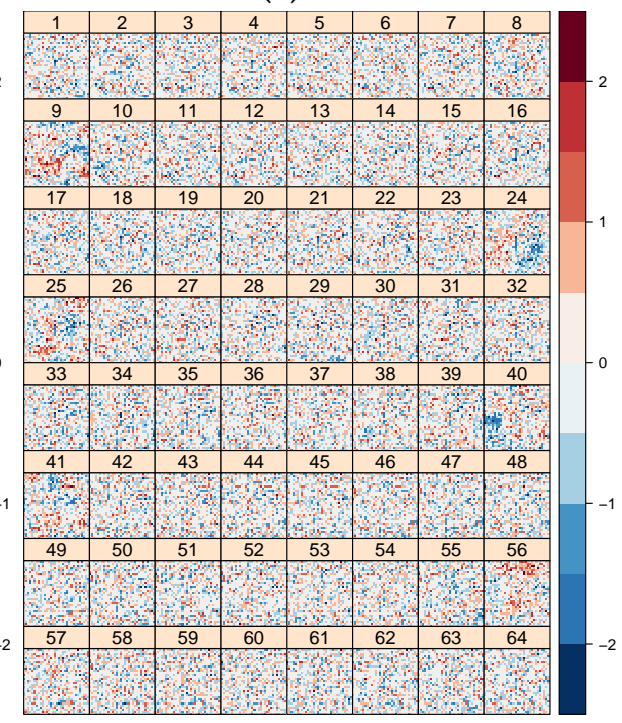

(f) Triangulação 2

Figura 5.8: Viés das predições para a Simulação apresentada na Figura 5.1, com os métodos descritos nessa seção, e marcado pelas cores indicadas na escala. 


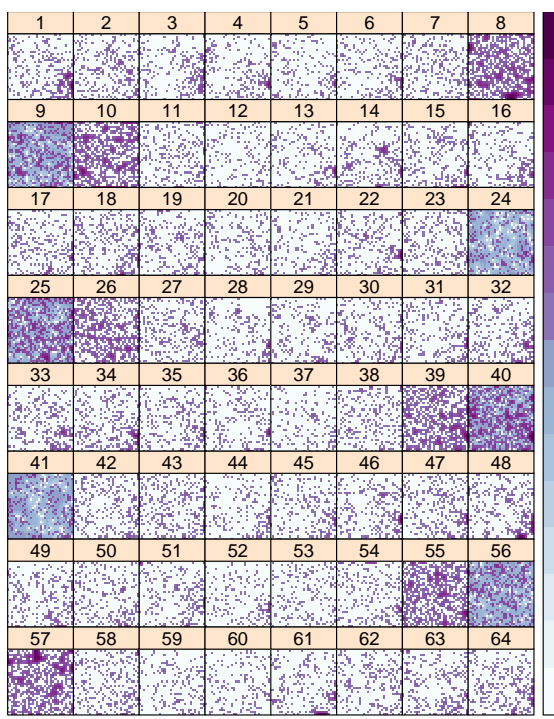

(a) Krigagem

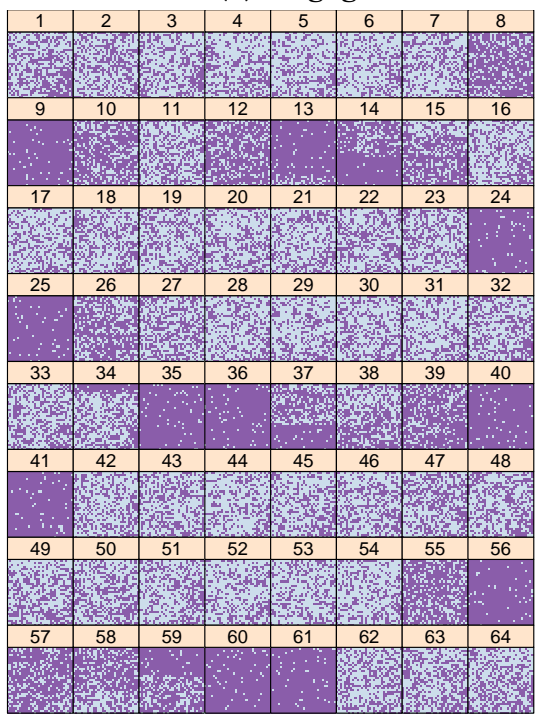

(c) FRK 2

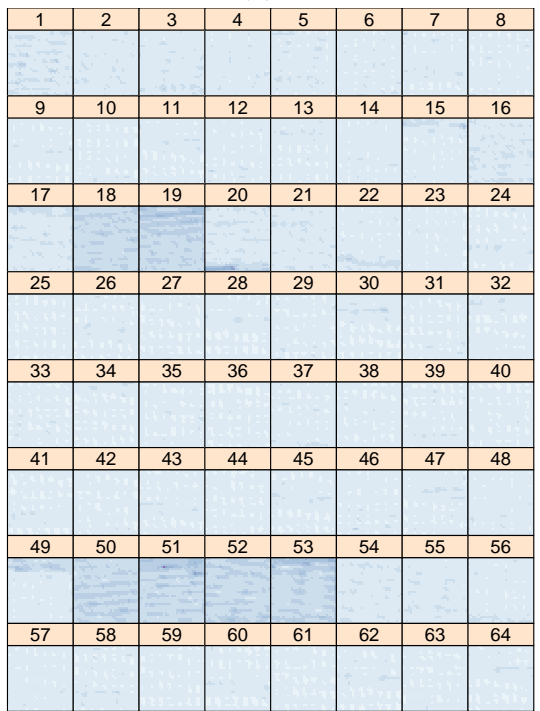

(e) Triangulação 1

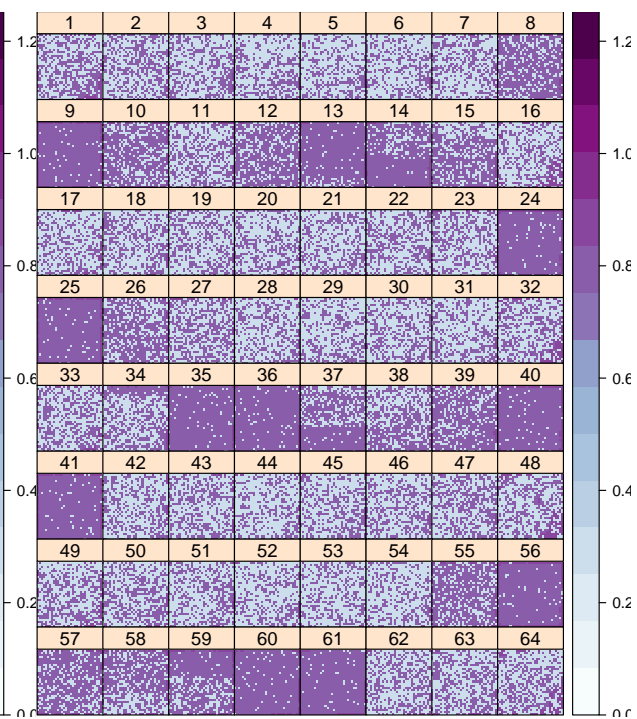

(b) FRK 1

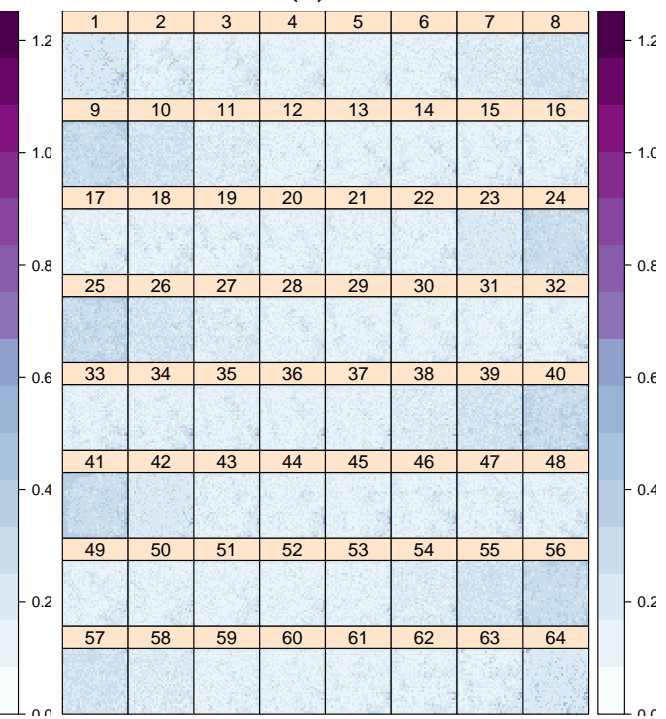

(d) $C A R$

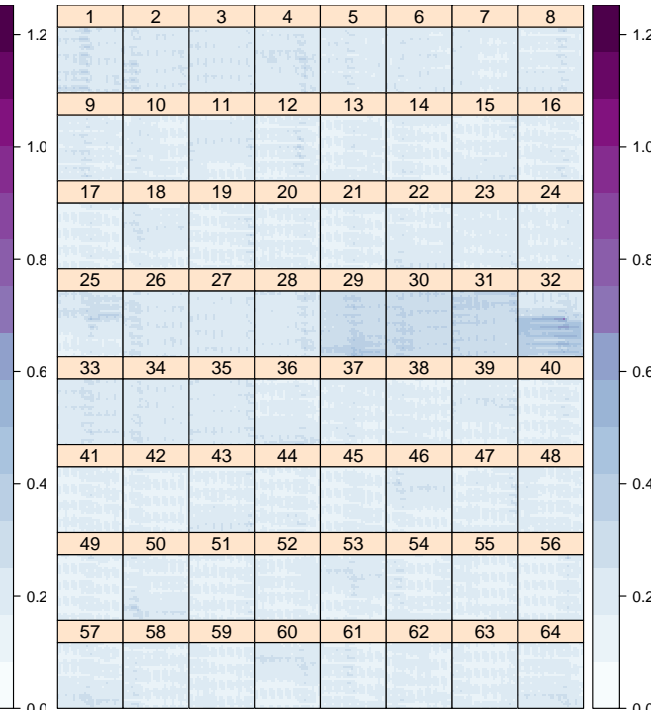

(f) Triangulação 2

Figura 5.9: Erro padrão estimado das predições para a Simulação apresentada na Figura 5.1, com os métodos descritos nessa seção. 


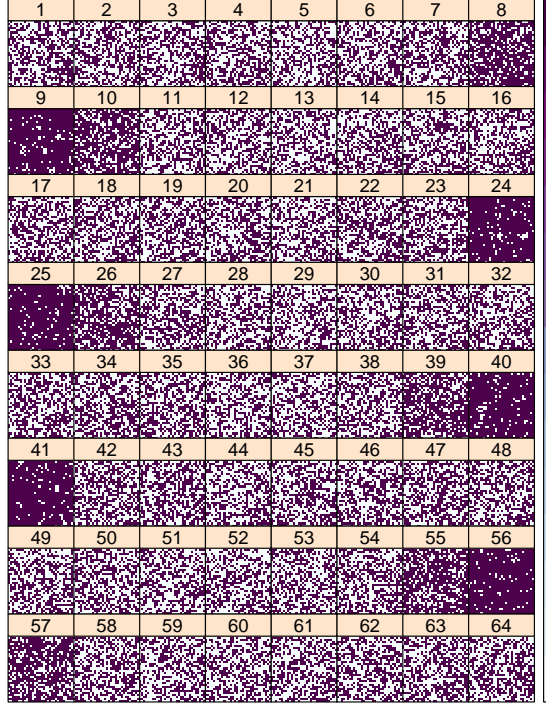

(a) Krigagem

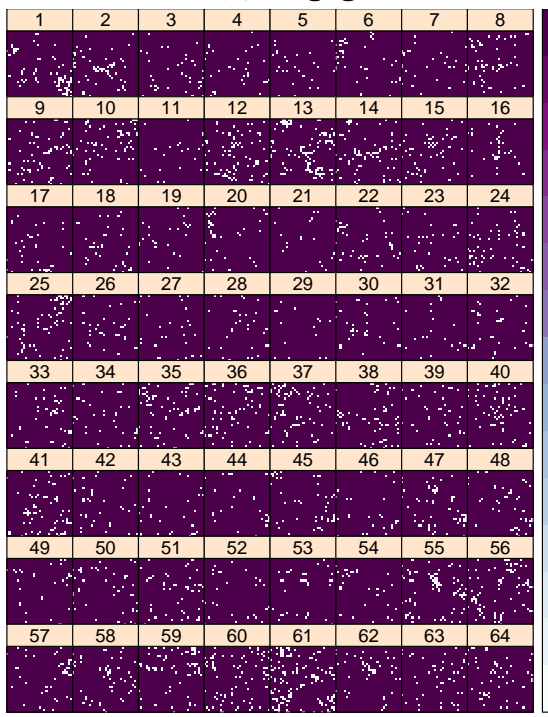

(c) FRK 2

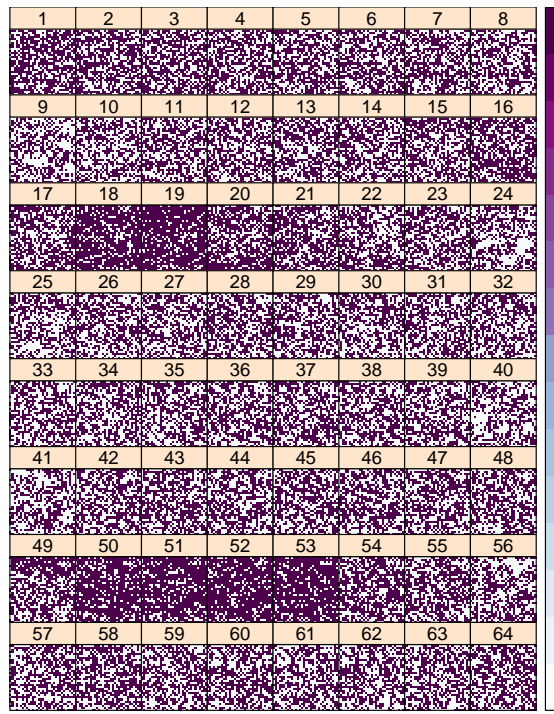

(e) Triangulação 1

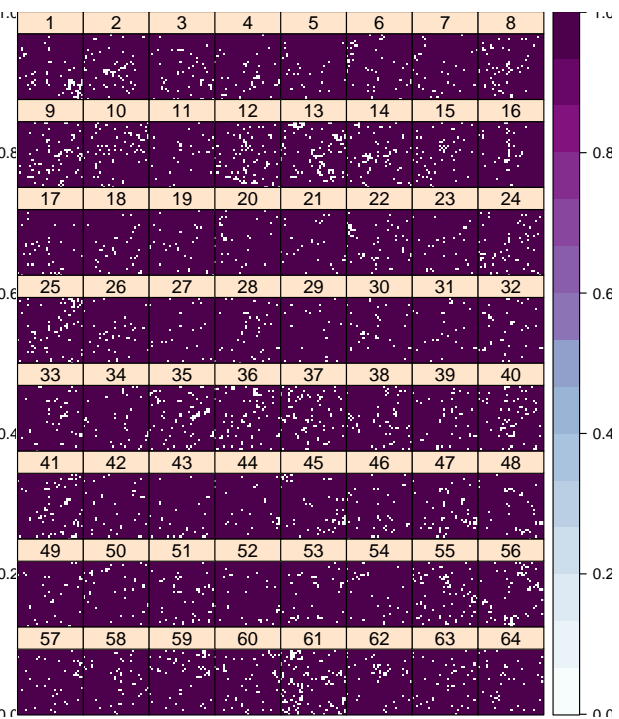

(b) FRK 1

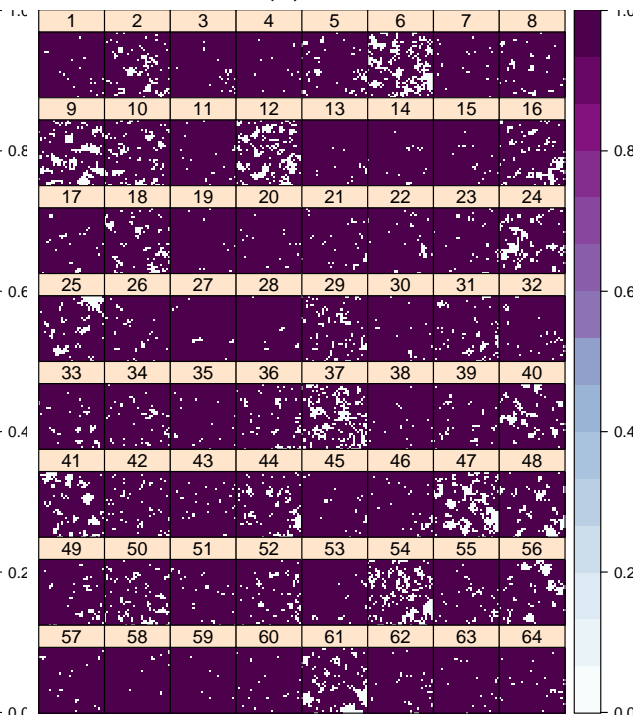

(d) $C A R$

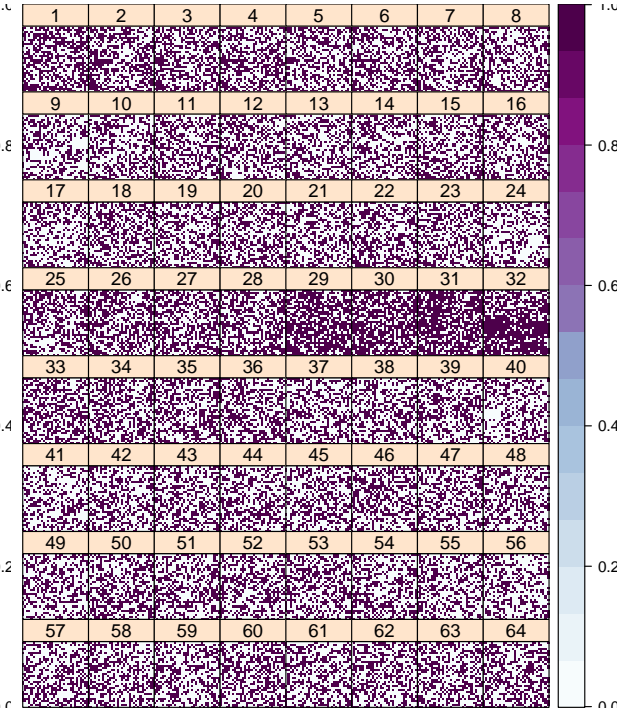

(f) Triangulação 2

Figura 5.10: Intervalo das predições para a validar a simulação apresentada na Figura 5.1, com os métodos descritos nessa seção. A cor roxa indica que o valor real para o ponto ficou dentro do intervalo de predição, e a cor branca indica que o valor real ficou fora do intervalo. 


\subsection{Resultados}

Para cada uma das 10 simulações, em suas formas originais (Conjunto 1) e acrescidas de um efeito sazonal (Conjunto 2) e para cada um dos métodos, foram calculados os erros quadráticos médios de predição. As Tabelas 5.1 e 5.2 apresentam o EQM para as predições de todos os pontos associados às observações. As Tabelas 5.3 e 5.4 apresentam o EQM para as predições associadas às observações mantidas no conjunto de dados. As Tabelas $5.5 \mathrm{e}$ 5.6 apresentam os erros quadráticos médios para as predições associadas às observações suprimidas do conjunto de dados.

A Figura 5.11 apresenta os boxplots dos erros quadráticos médios para as predições de todos os pontos associados às observações em cada um dos modelos testados. A Figura 5.12 apresenta os boxplots dos erros quadráticos médios para as predições associadas às observações mantidas no conjunto em cada um dos modelos testados. A Figura 5.13 apresenta os boxplots dos erros quadráticos médios para as predições associadas às observações suprimidas no conjunto em cada um dos modelos testados.

A Tabela 5.7 apresenta o tempo médio de execução das 10 simulações para os conjuntos 1 e 2 e para os seis métodos avaliados.

\subsection{Conclusões}

As Tabelas 5.1 a 5.6 e as Figuras 5.11 a 5.13 reforçam o que foi visto nas Figuras 5.4 a 5.7 de que o EQM é maior para os modelos FRK com os parâmetros escolhidos. Também é verificado maior variabilidade entre os EQMs para os modelos FRK, o que também pode ser visto nos EQMs por dia desses modelos, como no exemplo das Figuras 5.4 a 5.7.

No caso do conjunto de dados sazonais, para as observações que foram mantidas no conjunto, os modelos com redução de dimensionalidade por triangulação apresentaram EQM maiores. Para a Triangulação 1 (com mais triângulos) o EQM ficou próximo do EQM dos modelos FRK, enquanto que para a Triangulação 2 o EQM foi maior. No entanto, para as observações suprimidas, o EQM para as triangulações foi menor, sendo inclusive menor que o observado com a Krigagem.

O método que apresentou o menor EQM foi o CAR, tanto nos dados mantidos quanto nos suprimidos e para os Conjuntos 1 e 2, corroborando o que foi observado nas Figuras 5.4 a 5.7 para a simulação escolhida de exemplo e também os mapas de viés da Figura 5.8. No entanto, a Tabela 5.7 mostra que esse método é o que apresenta maior tempo médio de execução, sendo até 20 vezes mais demorado que as triangulações. 


\begin{tabular}{lccccc}
\hline \hline & FRK 1 & FRK 2 & CAR & Triangulação 1 & Triangulação 2 \\
\hline Sim. 1 & 0,922 & 0,779 & 0,090 & 0,285 & 0,447 \\
Sim. 2 & 0,860 & 0,748 & 0,059 & 0,282 & 0,422 \\
Sim. 3 & 0,864 & 0,712 & 0,081 & 0,283 & 0,425 \\
Sim. 4 & 0,861 & 0,745 & 0,071 & 0,274 & 0,427 \\
Sim. 5 & 0,866 & 0,785 & 0,065 & 0,288 & 0,428 \\
Sim. 6 & 0,905 & 0,774 & 0,088 & 0,284 & 0,427 \\
Sim. 7 & 0,875 & 0,748 & 0,065 & 0,286 & 0,439 \\
Sim. 8 & 0,906 & 0,767 & 0,083 & 0,292 & 0,437 \\
Sim. 9 & 0,918 & 0,753 & 0,068 & 0,290 & 0,423 \\
Sim. 10 & 0,905 & 0,110 & 0,294 & 0,427 & 0,427 \\
\hline
\end{tabular}

Tabela 5.1: Conjunto 1 - Erro quadrático médio da predição - Todas as observações

\begin{tabular}{lccccc}
\hline \hline & FRK 1 & FRK 2 & CAR & Triangulação 1 & Triangulação 2 \\
\hline Sim. 1 & 0,523 & 0,465 & 0,114 & 0,287 & 0,449 \\
Sim. 2 & 0,398 & 0,359 & 0,059 & 0,283 & 0,423 \\
Sim. 3 & 0,526 & 0,465 & 0,086 & 0,284 & 0,427 \\
Sim. 4 & 0,439 & 0,395 & 0,073 & 0,275 & 0,428 \\
Sim. 5 & 0,426 & 0,407 & 0,065 & 0,289 & 0,428 \\
Sim. 6 & 0,524 & 0,481 & 0,099 & 0,286 & 0,429 \\
Sim. 7 & 0,496 & 0,449 & 0,073 & 0,286 & 0,439 \\
Sim. 8 & 0,505 & 0,439 & 0,077 & 0,293 & 0,437 \\
Sim. 9 & 0,520 & 0,458 & 0,085 & 0,293 & 0,426 \\
Sim. 10 & 0,526 & 0,469 & 0,099 & 0,295 & 0,428 \\
\hline
\end{tabular}

Tabela 5.2: Conjunto 2 - Erro quadrático médio da predição - Todas as observações

\begin{tabular}{lccccc}
\hline \hline & FRK 1 & FRK 2 & CAR & Triangulação 1 & Triangulação 2 \\
\hline Sim. 1 & 0,850 & 0,713 & 0,023 & 0,245 & 0,400 \\
Sim. 2 & 0,820 & 0,712 & 0,031 & 0,255 & 0,388 \\
Sim. 3 & 0,805 & 0,667 & 0,025 & 0,234 & 0,366 \\
Sim. 4 & 0,823 & 0,712 & 0,031 & 0,242 & 0,385 \\
Sim. 5 & 0,827 & 0,749 & 0,028 & 0,252 & 0,391 \\
Sim. 6 & 0,839 & 0,719 & 0,034 & 0,238 & 0,372 \\
Sim. 7 & 0,828 & 0,708 & 0,020 & 0,245 & 0,388 \\
Sim. 8 & 0,866 & 0,734 & 0,037 & 0,254 & 0,390 \\
Sim. 9 & 0,868 & 0,708 & 0,015 & 0,244 & 0,374 \\
Sim. 10 & 0,838 & 0,696 & 0,028 & 0,250 & 0,374 \\
\hline
\end{tabular}

Tabela 5.3: Conjunto 1 - Erro quadrático médio - Observações mantidas no conjunto 


\begin{tabular}{lccccc}
\hline \hline & FRK 1 & FRK 2 & CAR & Triangulação 1 & Triangulação 2 \\
\hline Sim. 1 & 0,279 & 0,254 & 0,024 & 0,247 & 0,400 \\
Sim. 2 & 0,213 & 0,194 & 0,028 & 0,254 & 0,387 \\
Sim. 3 & 0,306 & 0,276 & 0,025 & 0,233 & 0,364 \\
Sim. 4 & 0,248 & 0,226 & 0,026 & 0,241 & 0,385 \\
Sim. 5 & 0,239 & 0,231 & 0,025 & 0,252 & 0,391 \\
Sim. 6 & 0,300 & 0,277 & 0,031 & 0,236 & 0,370 \\
Sim. 7 & 0,289 & 0,266 & 0,025 & 0,244 & 0,386 \\
Sim. 8 & 0,300 & 0,267 & 0,030 & 0,253 & 0,390 \\
Sim. 9 & 0,305 & 0,269 & 0,018 & 0,243 & 0,373 \\
Sim. 10 & 0,299 & 0,268 & 0,018 & 0,250 & 0,375 \\
\hline
\end{tabular}

Tabela 5.4: Conjunto 2 - Erro quadrático médio - Observações mantidas no conjunto

\begin{tabular}{lcccccc}
\hline \hline & Krigagem & FRK 1 & FRK 2 & CAR & Triangulação 1 & Triangulação 2 \\
\hline Sim. 1 & 0,692 & 1,083 & 0,926 & 0,239 & 0,373 & 0,550 \\
Sim. 2 & 0,628 & 0,990 & 0,864 & 0,149 & 0,369 & 0,533 \\
Sim. 3 & 0,580 & 0,979 & 0,799 & 0,190 & 0,379 & 0,540 \\
Sim. 4 & 0,562 & 0,961 & 0,834 & 0,178 & 0,360 & 0,536 \\
Sim. 5 & 0,524 & 0,973 & 0,887 & 0,167 & 0,387 & 0,529 \\
Sim. 6 & 0,605 & 1,045 & 0,892 & 0,205 & 0,384 & 0,543 \\
Sim. 7 & 0,596 & 0,982 & 0,837 & 0,167 & 0,377 & 0,552 \\
Sim. 8 & 0,584 & 1,003 & 0,843 & 0,192 & 0,381 & 0,546 \\
Sim. 9 & 0,556 & 1,025 & 0,849 & 0,180 & 0,388 & 0,527 \\
Sim. 10 & 0,585 & 1,040 & 0,864 & 0,277 & 0,385 & 0,534 \\
\hline
\end{tabular}

Tabela 5.5: Conjunto 1 - Erro quadrático médio da predição - Observações removidas no conjunto

\begin{tabular}{ccccccc}
\hline \hline & Krigagem & FRK 1 & FRK 2 & CAR & Triangulação 1 & Triangulação 2 \\
\hline Sim. 1 & 0,696 & 1,032 & 0,905 & 0,301 & 0,370 & 0,549 \\
Sim. 2 & 0,622 & 0,956 & 0,855 & 0,150 & 0,369 & 0,532 \\
Sim. 3 & 0,579 & 0,924 & 0,805 & 0,197 & 0,376 & 0,541 \\
Sim. 4 & 0,574 & 0,923 & 0,822 & 0,191 & 0,360 & 0,535 \\
Sim. 5 & 0,523 & 0,914 & 0,867 & 0,170 & 0,384 & 0,526 \\
Sim. 6 & 0,611 & 0,974 & 0,891 & 0,236 & 0,387 & 0,549 \\
Sim. 7 & 0,589 & 0,929 & 0,832 & 0,173 & 0,373 & 0,551 \\
Sim. 8 & 0,580 & 0,962 & 0,823 & 0,180 & 0,380 & 0,542 \\
Sim. 9 & 0,563 & 0,951 & 0,839 & 0,220 & 0,394 & 0,532 \\
Sim. 10 & 0,590 & 0,962 & 0,855 & 0,253 & 0,383 & 0,531 \\
\hline
\end{tabular}

Tabela 5.6: Conjunto 2 - Erro quadrático médio da predição - Observações removidas no conjunto 


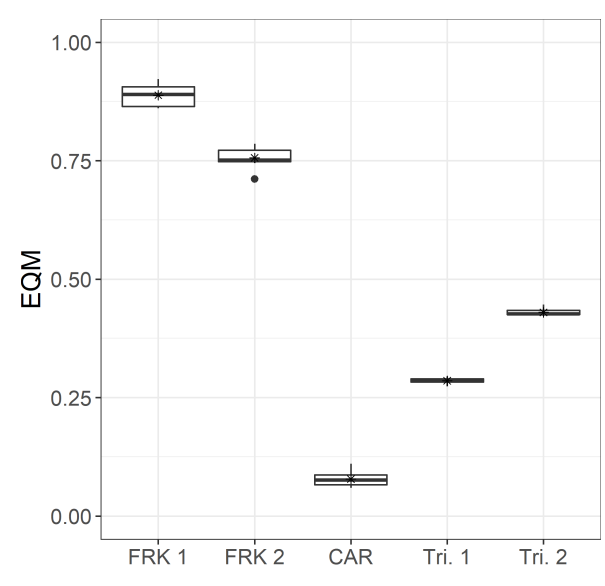

(a) Conjunto 1

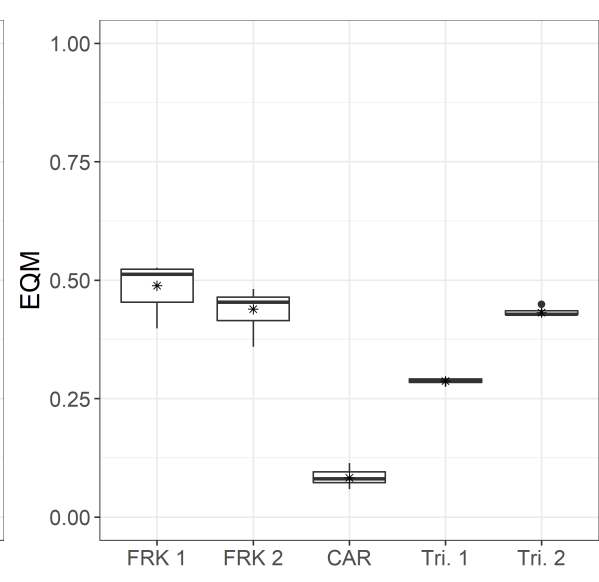

(b) Conjunto 2

Figura 5.11: Boxplots com os dados apresentados nas Tabelas 5.1 e 5.2: EQM - predições para todas as observações do conjunto de dados.

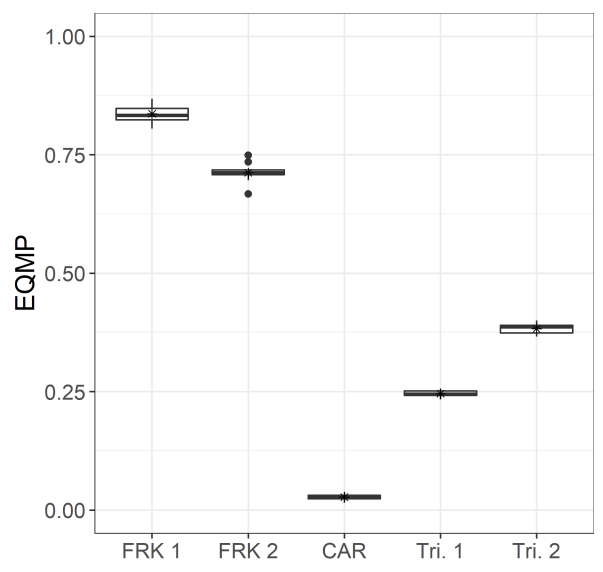

(a) Conjunto 1

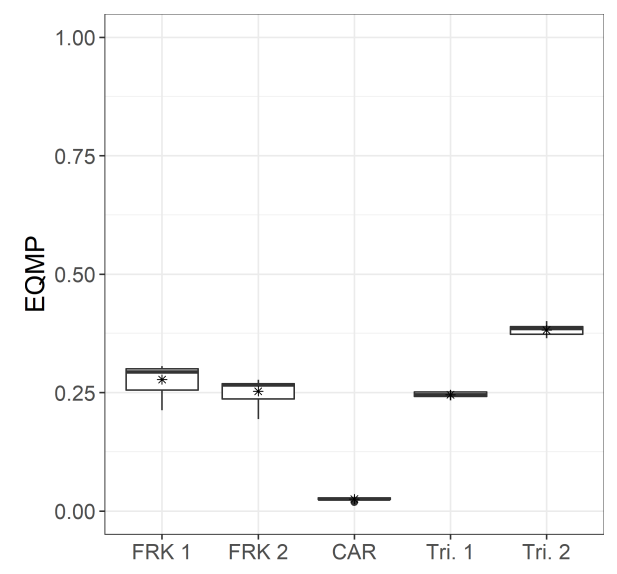

(b) Conjunto 2

Figura 5.12: Boxplots com os dados apresentados nas Tabelas 5.3 e 5.4: EQM - predições associadas às observações mantidas no conjunto de dados.

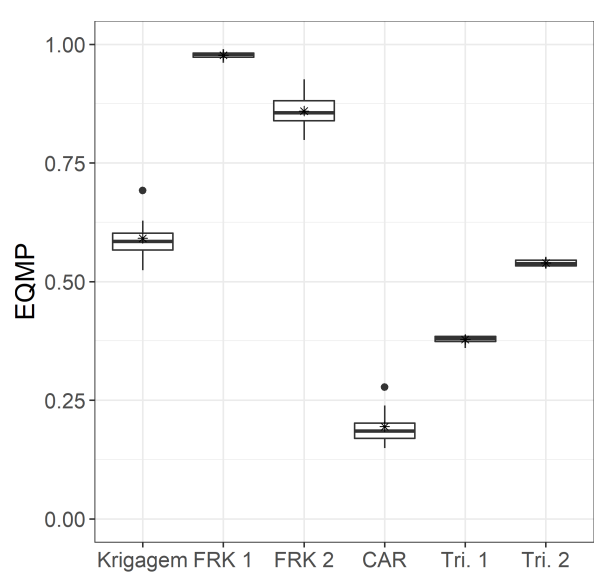

(a) Conjunto 1

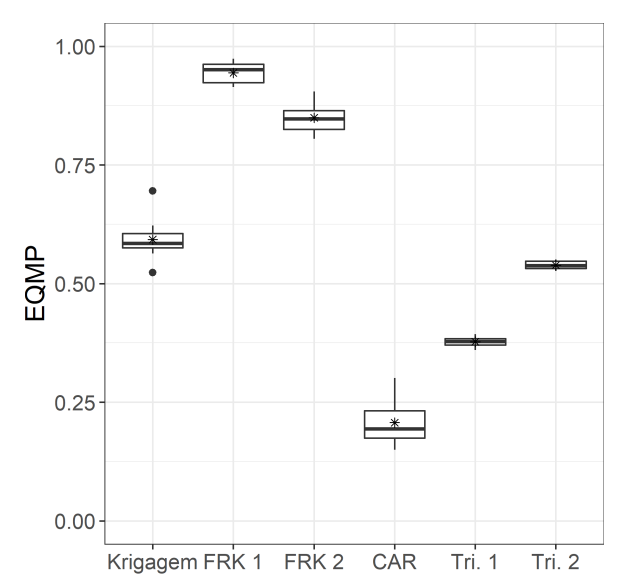

(b) Conjunto 2

Figura 5.13: Boxplots com os dados apresentados nas Tabelas 5.5 e 5.6: EQM - predições associadas às observações suprimidas do conjunto de dados. 
5.6 | CONCLUSÕES

\begin{tabular}{lcccccc}
\hline \hline & Krigagem & FRK 1 & FRK 2 & CAR & Triangulação 1 & Triangulação 2 \\
\hline Conjunto 1 & 58 & 40 & 48 & 103 & 27 & 7 \\
Conjunto 2 & 47 & 65 & 71 & 194 & 56 & 9 \\
\hline
\end{tabular}

Tabela 5.7: Tempo, em minutos, para a modelagem e a predição para o conjunto 1 (Sem sazonalidade) e o conjunto 2 (com sazonalidade) 



\section{Capítulo 6}

\section{Aplicação}

Desde que conjuntos de dados oriundos de sensoriamento remoto passaram a ser utilizados em pesquisas científicas, a krigagem foi bastante utilizada para a modelagem desses dados, notando-se o problema de complexidade computacional do método. Novos métodos já estão sendo empenhados em pesquisas científicas para viabilizar a interpolação em conjuntos espaço-temporais. ZAMMIT-MANGION e CRESSIE (2021) exemplificaram a implementação do método FRK com um conjunto de dados obtidos de um satélite com sensor para a coluna de dióxido de carbono medida em toda a superfície terrestre por 16 dias. CAmeletti et al. (2013) utilizaram o método para o modelo hierárquico espaçotemporal delineado no Capítulo 4 e a implementação do método INLA para obter predições para a concentração de material particulado (particulate matter ou PM) na região italiana de Piemonte, com dados obtidos de estações medidoras. Pu e Yoo (2019) avaliaram o método INLA para modelos usando um conjunto espaço-temporal com dados faltantes de partículas de poluição em Pequim.

Motivados pelas aplicações recentes para esses métodos, neste trabalho utilizamos um conjunto de dados obtidos por satélite para aplicar os métodos descritos nos Capítulos $3 \mathrm{e}$ 4 e simulados no Capítulo 5.

\subsection{Descrição dos Dados}

Foram utilizadas imagens orbitais fornecidas pelo Moderate Resolution Imaging Spectroradiometer (MODIS), sistema de observação terrestre desenvolvido pela National Aeronautics and Space Administration (NASA) e projetado para medir os processos físicos e biológicos diariamente e em escala global, com o propósito de compreender a dinâmica e os processos que ocorrem na superfície da Terra e na atmosfera inferior. O primeiro sensor foi montado na plataforma Terra da NASA em 1999, fornecendo produtos MODIS desde 2000. O segundo sensor foi lançado em 2002 a bordo da plataforma Aqua (WAN et al., 2015). Uma característica importante do MODIS é a correção dos efeitos atmosféricos (nuvens, aerossóis, etc.) e a disponibilidade de dados georreferenciados. Os dados podem ser obtidos através do sistema EarthData (ESDIS, 2019). 
Um produto do sensoriamento orbital produzido pelo MODIS é a imagem que fornece a Temperatura de Superfície Terrestre (LST, do inglês Land Surface Temperature) para uma área selecionada, em projeção sinusoidal. Nesse caso, cada pixel conterá a temperatura (em escala Kelvin) da região de $1 \mathrm{~km}^{2}$ que ele contempla. Este produto também fornece um conjunto de imagens que associa a cada pixel um indicador do erro estimado da medição. Detalhes sobre como esses valores são calculados estão disponíveis em WAN (2013).

Alguns produtos oferecidos pela plataforma EarthData calculam uma média de um intervalo de dias, oferecendo um conjunto com menos dados faltantes, mas com uma resolução temporal maior. Ainda assim, há muitos casos em que não é possível obter uma medição para a temperatura de superfície, devido à presença de nuvens espessas, aerossóis e outros artefatos na atmosfera.

Para este trabalho, foi utilizado o produto MYD11A2 (WAN et al., 2015), que fornece imagens com resolução temporal de 8 dias. Um subconjunto referente ao período entre julho de 2009 até junho de 2019 foi definido e dele foram extraídas 12 imagens por ano, referentes ao inicio de cada mês, totalizando 120 imagens.

A área selecionada para o recorte inclui integralmente as zonas central e oeste do município de São Paulo e os municípios de Osasco, Carapicuíba e Taboão da Serra, e apresenta também alguns distritos das zonas norte e sul de São Paulo. Esses distritos apresentam diferentes perfis de ocupação do solo e densidades populacionais, com regiões mais arborizadas e outras com cobertura verde reduzida. A Tabela 6.2 apresenta os distritos incluídos por inteiro na área selecionada e dados demográficos, calculados por zona da região escolhida.

A fim de aumentar a variedade de perfis de ocupação do solo na região selecionada, áreas de maior quantidade de vegetação ao norte da capital paulista e nos municípios de Cotia, Embu e Santana de Parnaíba foram incluídas no conjunto de dados para a análise. Partes dessas áreas passaram por mudanças na ocupação no período selecionado, com obras como o Rodoanel e a construção de condomínios residenciais e industriais. A Tabela 6.1 apresenta os limites da área selecionada e as Figuras 6.1 e 6.2 ilustram a área escolhida.

Tendo sido definida a área de recorte dos dados, resulta com conjunto de 120 imagens de $27 x 27$ pixels, totalizando 87.480 observações indexadas no tempo.

\begin{tabular}{cc}
\hline \hline Latitude & Longitude \\
\hline$-46,8197$ & -23.4181 \\
$-46,9021$ & -23.6423 \\
$-46,6582$ & -23.6413 \\
$-46,5745$ & -23.4172 \\
\hline
\end{tabular}

Tabela 6.1: As coordenadas geográficas dos pontos que delimitam a área selecionada. 


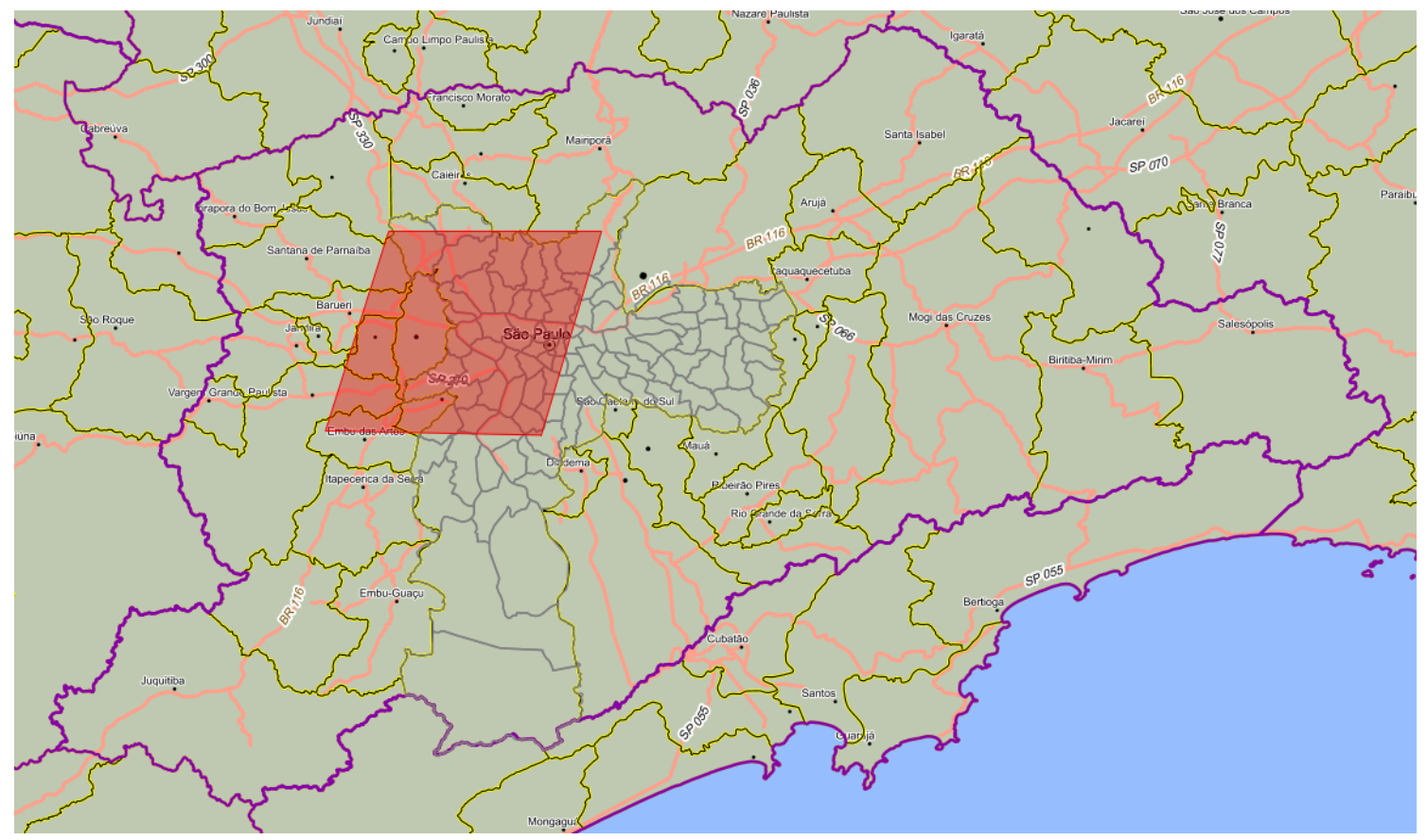

Figura 6.1: Localização da área utilizada na análise na Região Metropolitana de São Paulo (EMPLASA, 2019).

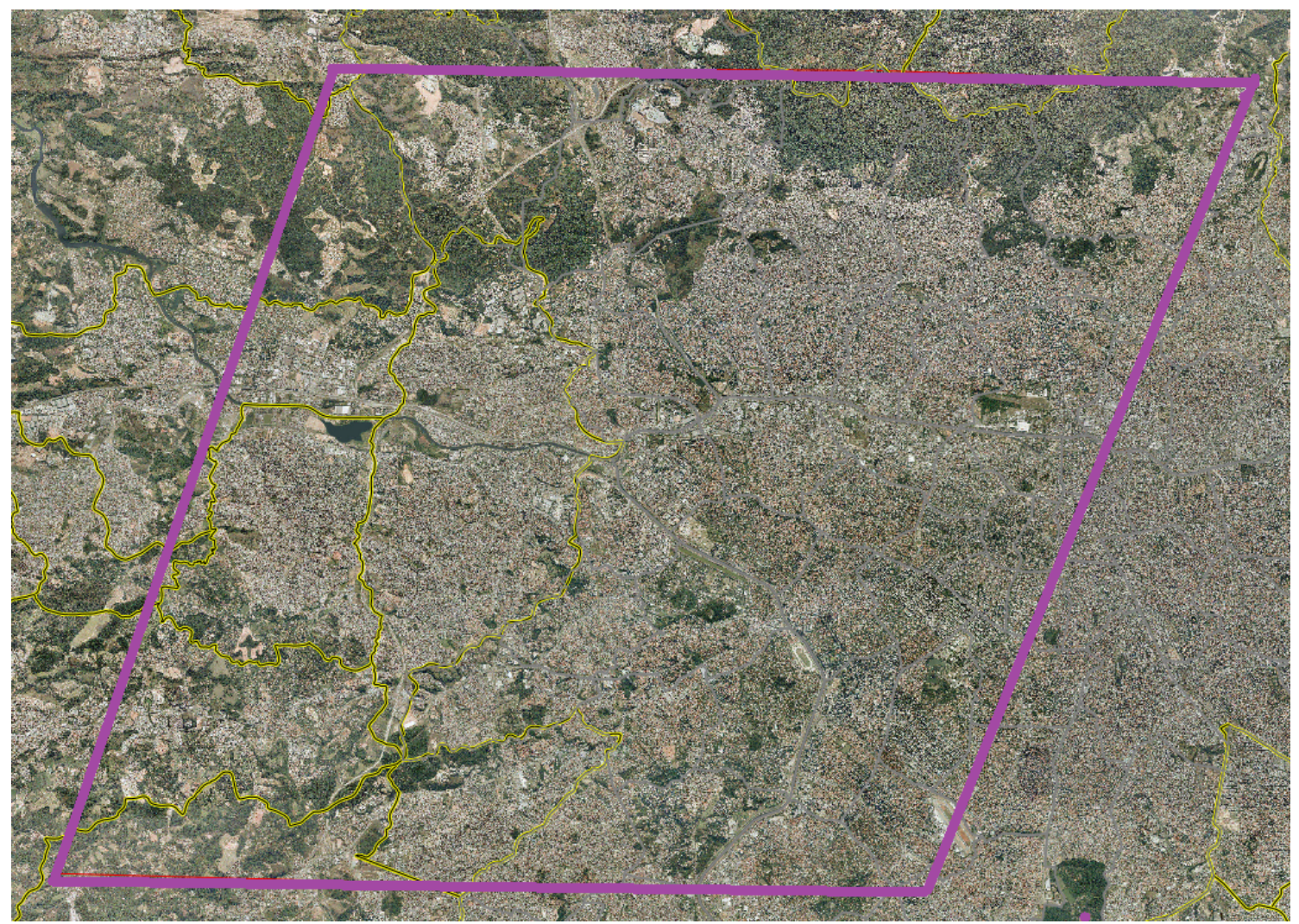

Figura 6.2: Ortofoto com a área utilizada no estudo em destaque (EMPLASA, 2010). 


\begin{tabular}{|c|c|c|c|c|}
\hline Zona & Distritos & Pop. & Área $\left(K m^{2}\right)$ & Dens. \\
\hline Centro & $\begin{array}{l}\text { Bela Vista, Bom Retiro, } \\
\text { Consolação, Liberdade, } \\
\text { Santa Cecília, Sé }\end{array}$ & 345.510 & 22,64 & $15.245,47$ \\
\hline Norte & $\begin{array}{l}\text { Brasilândia, Cachoeirinha, } \\
\text { Casa Verde, Freguesia do Ó, Jaraguá, } \\
\text { Limão, Mandaqui, Pirituba, } \\
\text { Santana, São Domingos, Tucuruvi }\end{array}$ & 1.423 .239 & 151,28 & $9.408,25$ \\
\hline Oeste & $\begin{array}{l}\text { Alto de Pinheiros, Barra Funda, } \\
\text { Butantã, Jaguara, Jaguaré, } \\
\text { Jardim Paulista, Lapa, Perdizes, } \\
\text { Pinheiros, Raposo Tavares, } \\
\text { Rio Pequeno, Vila Leopoldina }\end{array}$ & 717.383 & 97,53 & $7.355,35$ \\
\hline Sul & $\begin{array}{l}\text { Campo Belo, Itaim Bibi, Moema, } \\
\text { Morumbi, Vila Andrade, Vila Sônia }\end{array}$ & 414.994 & 59,94 & $6.924,01$ \\
\hline $\begin{array}{c}\text { Outros } \\
\text { Municípios }\end{array}$ & Carapicuíba, Osasco, Taboão da Serra & 1.194 .833 & 119,19 & $10.024,91$ \\
\hline
\end{tabular}

Tabela 6.2: Dados demográficos da área selecionada, por zona considerando os distritos incluídos por inteiro no mapa (IBGE, 2002).

\subsection{Análise Descritiva}

Ilustramos na Figura 6.3 a sequência de mapas obtidos após o recorte da área, com as temperaturas indicadas pelas cores dos pontos, em escala Kelvin. Observamos que há grande quantidade de pontos faltantes em alguns períodos específicos (principalmente nos meses mais quentes). A Figura 6.4 apresenta a série temporal com a quantidade de dados que foram observados, por imagem. Observamos que há muitas imagens completas, porém há duas em que nenhuma observação foi adquirida (Estas se referem à sexta e à septuagésima-sétima imagem incluída na análise, relacionadas a dezembro de 2009 e a novembro de 2015, respectivamente). Também verificamos maior quantidade de dados faltantes relacionadas às áreas que representam as localidades mais densamente povoadas da região escolhida.

A Figura 6.5 apresenta quatro séries temporais relacionadas à áreas com diferentes perfis de ocupação do solo, para fins de ilustração. Nas figuras desta seção, verificamos a existência de um efeito sazonal nos dados observados, o que é esperado para um fenômeno climático. 


\section{Dados - Jul/09 - Jun/19}

\begin{tabular}{|c|c|c|c|c|c|c|c|c|c|c|c|}
\hline 1 & 2 & 3 & 4 & 5 & 6 & 7 & 8 & 9 & 10 & 11 & 12 \\
\hline & 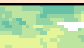 & & & & & & & & & & \\
\hline 13 & 14 & 15 & 16 & 17 & 18 & 10 & 20 & 21 & 20 & 23 & 21 \\
\hline$x_{1}$ & 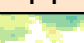 & & & & & & \begin{tabular}{|l|l} 
\\
\end{tabular} & 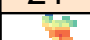 & & & 27 \\
\hline 25 & 26 & 27 & 28 & 29 & 30 & 31 & 32 & 33 & 34 & 35 & 36 \\
\hline & 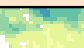 & & 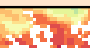 & & & & 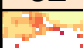 & & & 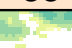 & \\
\hline & $y=$ & & +7 & & 1 & & & & 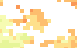 & 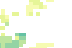 & 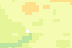 \\
\hline 37 & 38 & 39 & 40 & 41 & 42 & 43 & 44 & 45 & 46 & 47 & 48 \\
\hline & & & & & & & & & & & \\
\hline 49 & 50 & 51 & 52 & 53 & 54 & 55 & 56 & 57 & 58 & 59 & 60 \\
\hline & 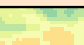 & & $r$ & & & & & & & & 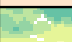 \\
\hline 61 & 62 & 63 & 64 & 65 & 66 & 67 & 68 & 69 & 70 & 71 & 72 \\
\hline$\theta_{0}$ & & 8 & 4 & & & . & & & & का & \\
\hline H & & & & & & & & & & & \\
\hline 73 & 74 & 75 & 76 & 77 & 78 & 79 & 80 & 81 & 82 & 83 & 84 \\
\hline$y_{1}$ & & & 5 & & & & $\therefore$ & & & & \\
\hline 85 & 86 & 87 & 88 & 89 & 90 & 91 & 92 & 93 & 94 & 95 & 96 \\
\hline & 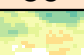 & & $x^{2}$ & & & & & & 8 & 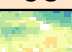 & \\
\hline 97 & תחת & 00 & 100 & 101 & 100 & 100 & 101 & 105 & 100 & 107 & 100 \\
\hline प्र1 & 98 & 99 & 100 & 101 & 102 & 103 & 104 & 105 & 106 & / & 108 \\
\hline 3 & & 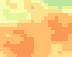 & $4 y^{2}$ & & & & & & & 4 & $=$ \\
\hline 109 & $\frac{}{110}$ & 111 & $\frac{1}{112}$ & 113 & 114 & 115 & 116 & $\frac{1}{117}$ & 110 & 110 & $=$ \\
\hline 109 & 110 & 111 & 112 & & 114 & 115 & 110 & 117 & 118 & 119 & 120 \\
\hline & & & & & & & & & & & \\
\hline
\end{tabular}

Figura 6.3: Imagens que representam a temperatura de superfície (K) nas áreas da região selecionada. A imagem 1 representa o mês de julho de 2009 e cada imagem representa um mês.

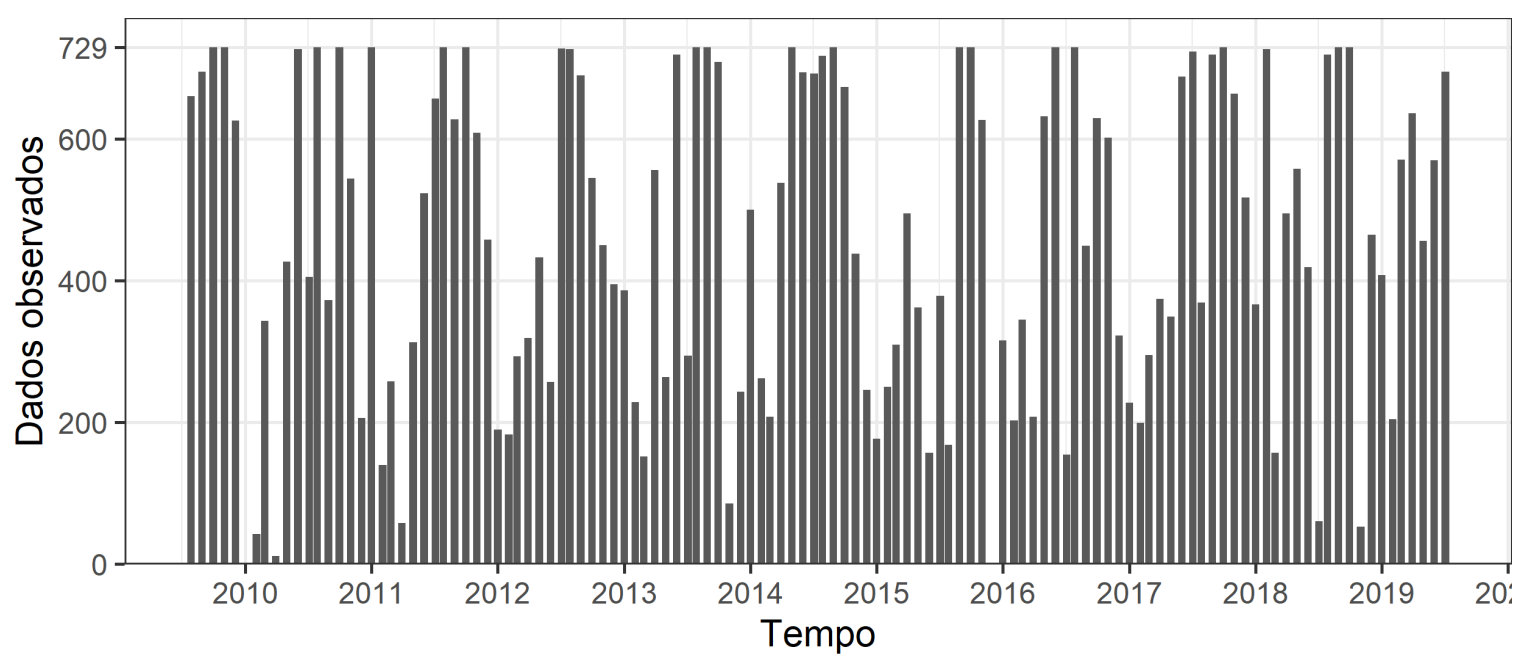

Figura 6.4: Quantidade de áreas com valores observados, por imagem. 


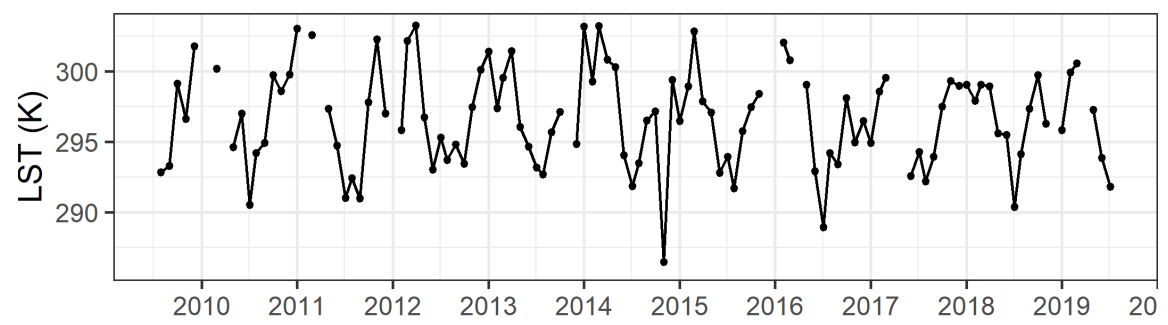

(a) Área de mata no distrito da Brasilândia

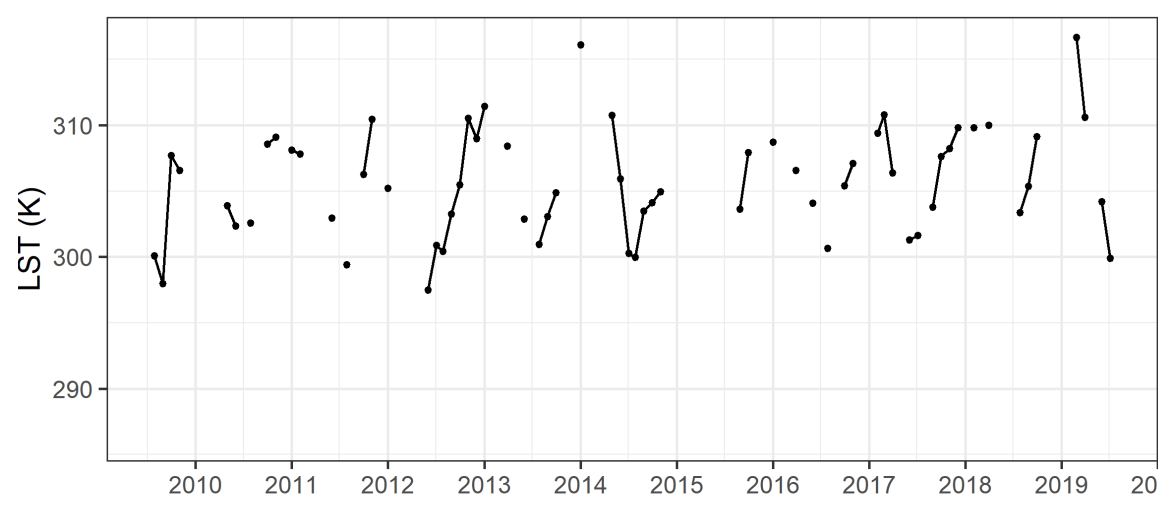

(b) Área densamente ocupada no município de Carapicuíba

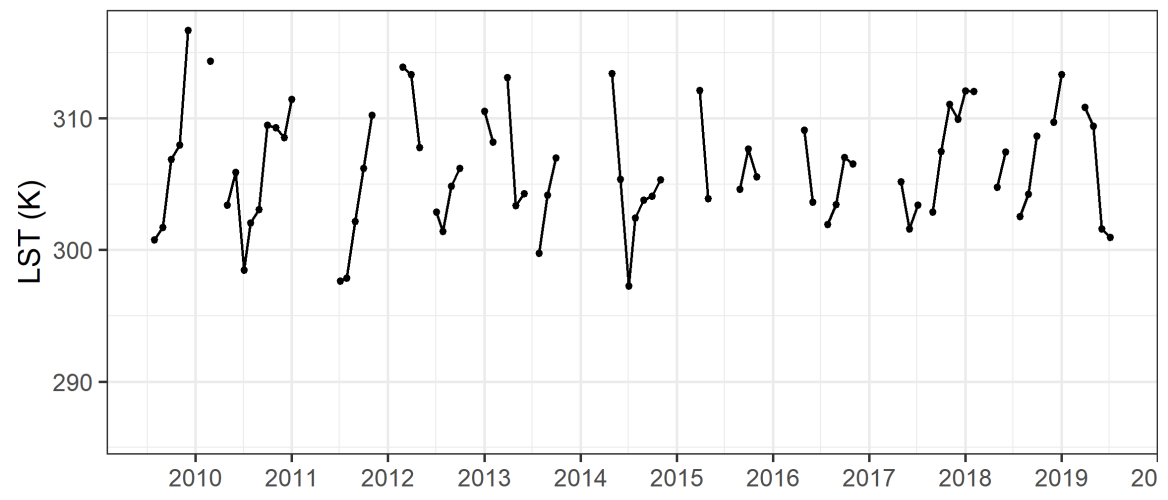

(c) Área da região da Sé, centro de São Paulo

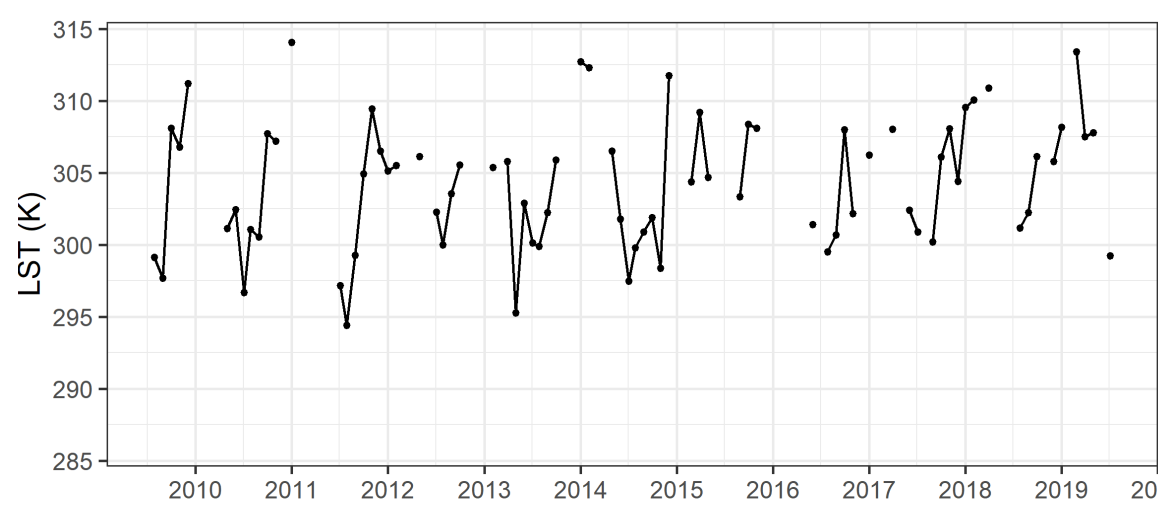

(d) Área do distrito de Moema, São Paulo, incluindo parte do Parque do Ibirapuera

Figura 6.5: Gráficos das séries temporais de temperatura de superfície para quatro áreas de $1 \mathrm{Km}^{2}$ dentre as 729 que fazem parte do conjunto de dados selecionado. 
6.3 | PREDIÇÕES

\subsection{Predições}

A Figura 6.6 apresenta os mapas resultantes das predições utilizando os quatro métodos avaliados: krigagem universal, Krigagem de Posto Fixo (FRK), Autorregressivo Condicional (CAR) e Triangulação-INLA. Foi escolhido um conjunto de 90 bases espaciais e 20 bases temporais para o método FRK e uma triangulação de 1,5 vértices para cada área observada para a triangulação. A Figura 6.7 apresenta os erros padrões estimados.

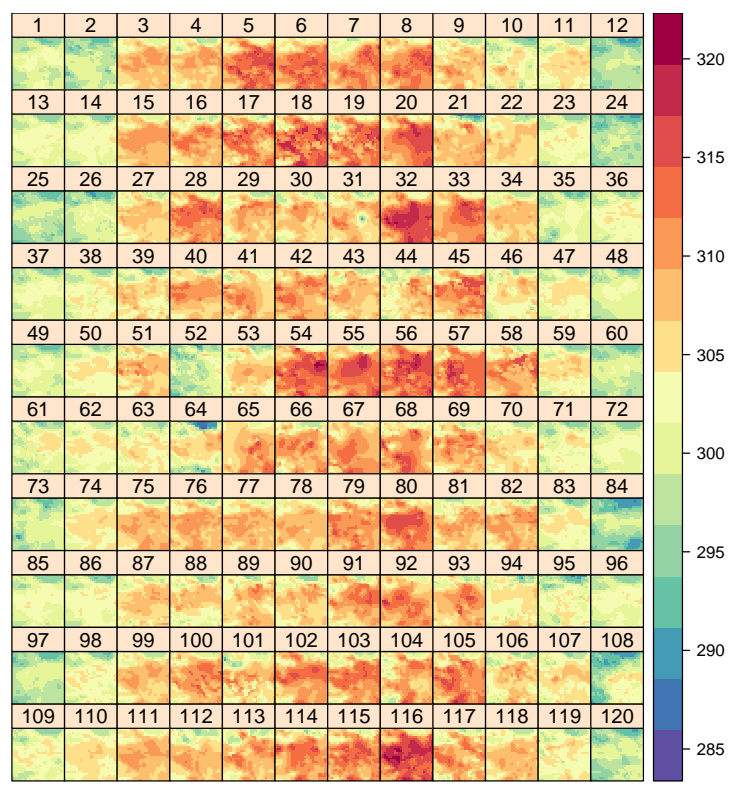

(a) Krigagem

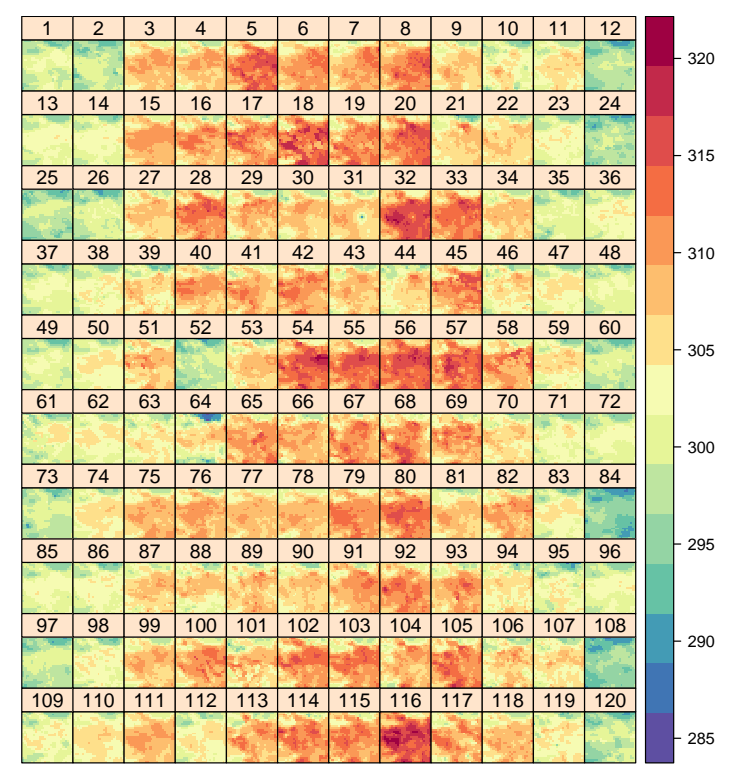

(c) $C A R$

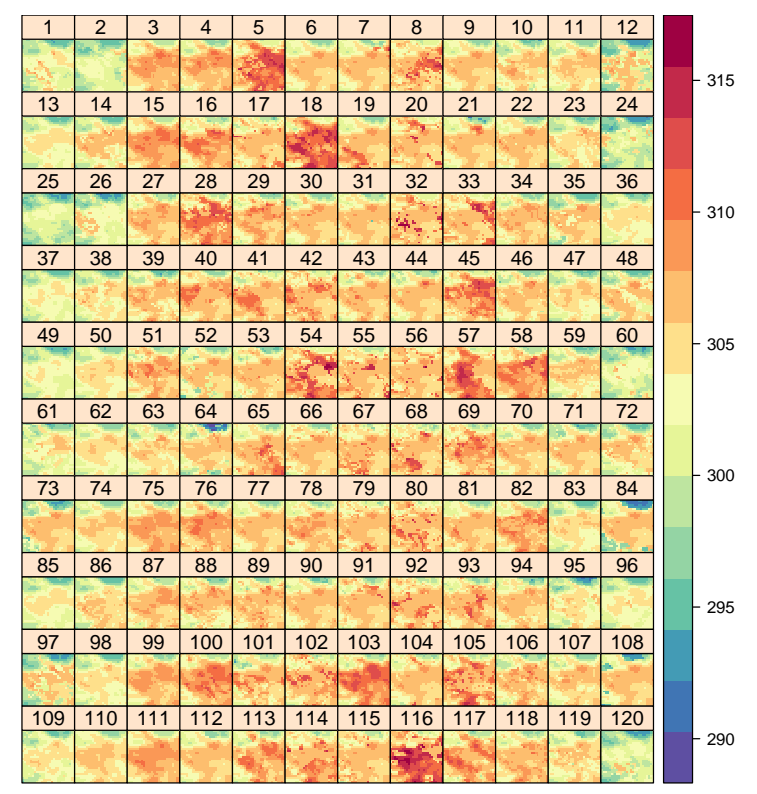

(b) FRK

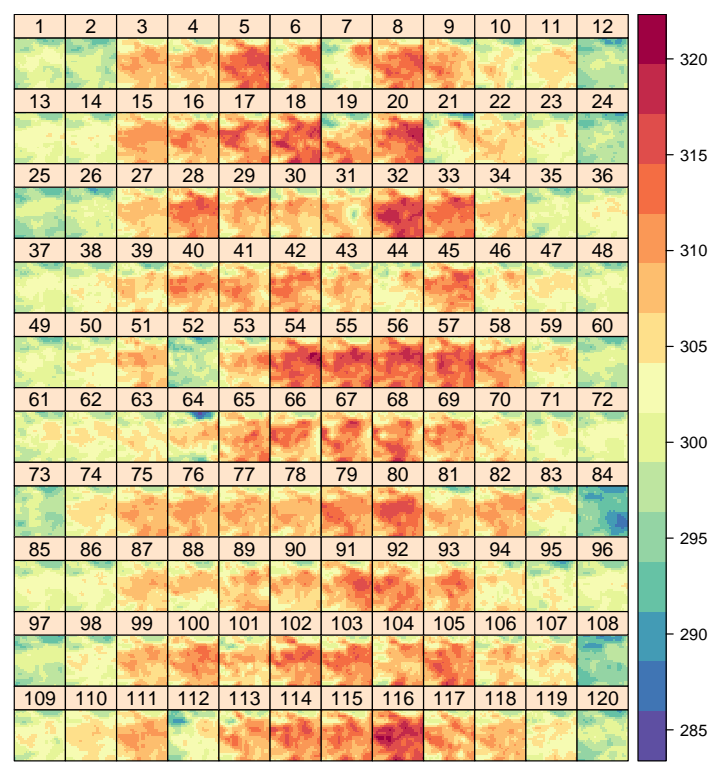

(d) Triangulação

Figura 6.6: Predições para preencher o conjunto de dados apresentado na Figura 6.3, para cada um dos métodos avaliados. 


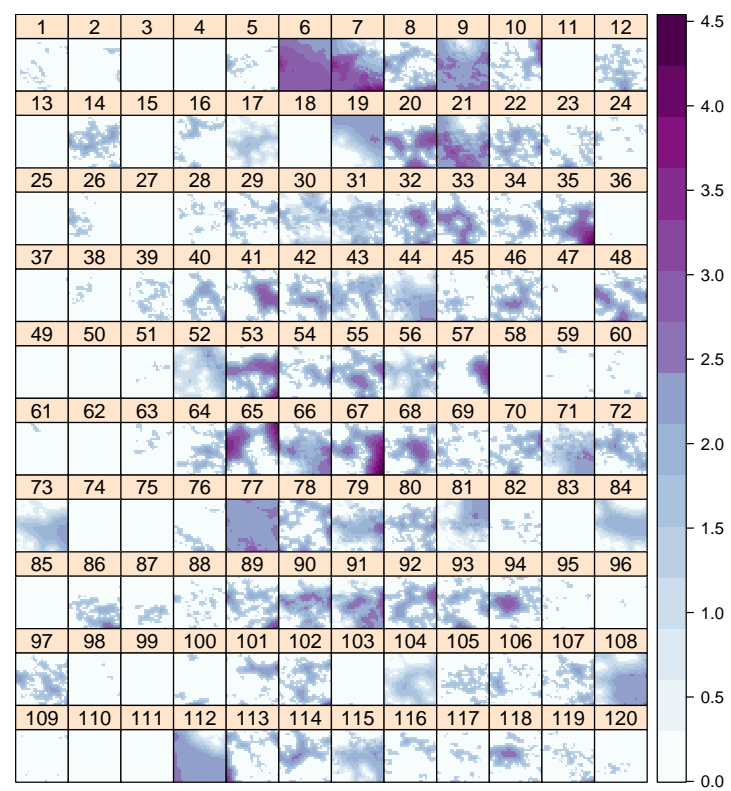

(a) Krigagem

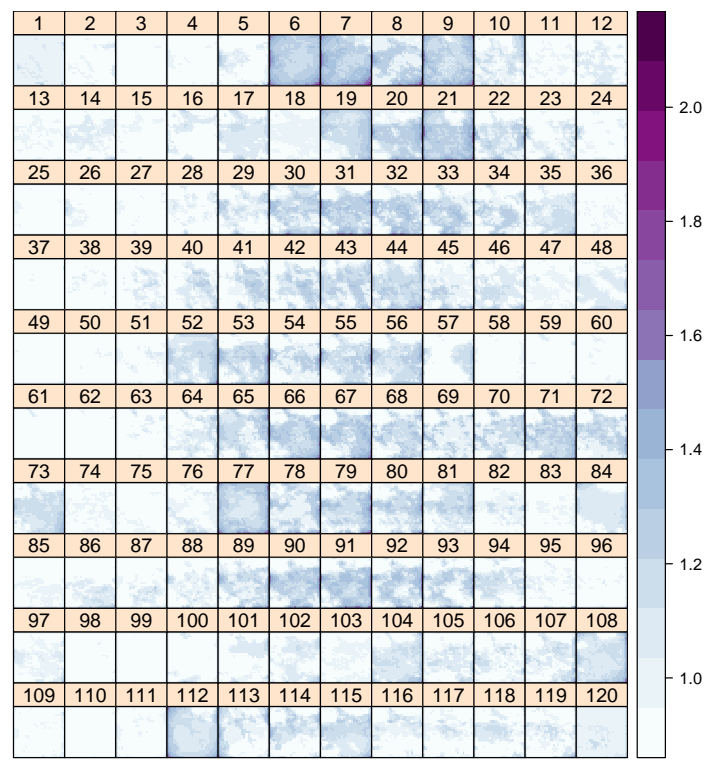

(c) $C A R$

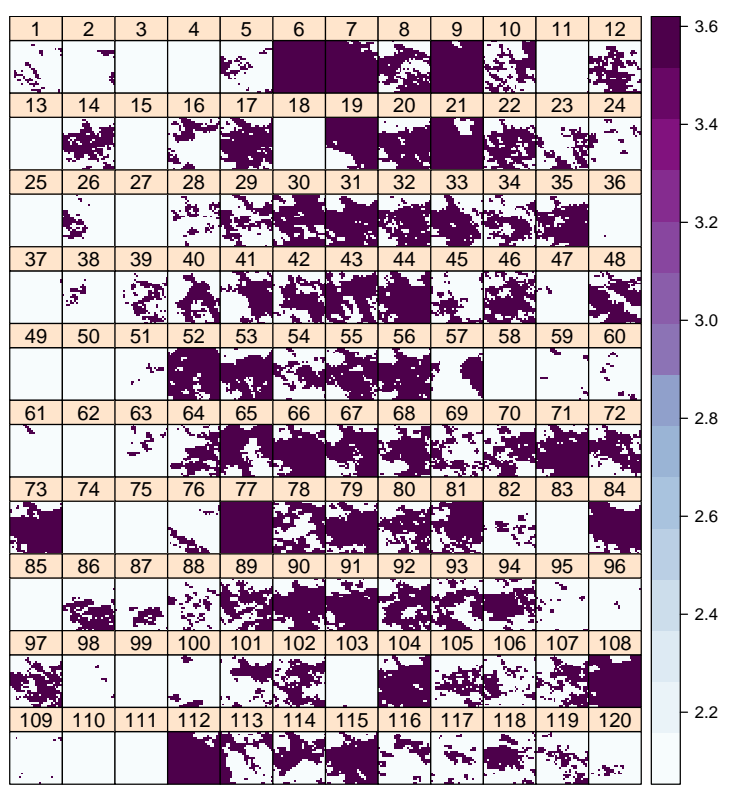

(b) FRK

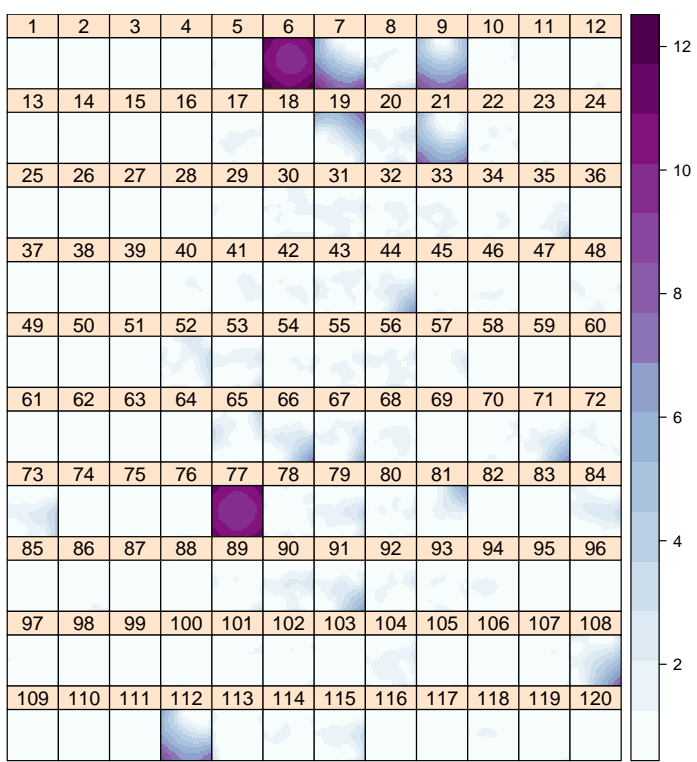

(d) Triangulação

Figura 6.7: Erros padrões estimados para a predição de cada localidade do conjunto de dados. Nota-se a diferença nas escalas. 
Como observado no Capítulo 5, o método FRK apresenta predições mais suavizadas quando comparado com os métodos CAR e a Triangulação escolhida, uma consequência do uso de funções-base para a redução de dimensionalidade que resulta em uma possível perda de detalhes nas imagens. Já o erro padrão estimado tende a ser maior especialmente para o método da triangulação nas datas em que não havia nenhum ponto disponível.

Na Figura 6.8 observamos que para essa aplicação os valores preditos pelo método CAR ficaram bem mais próximos aos valores reais do que os valores preditos pelos métodos Triangulação e FRK. O método FRK aparenta estar menos disperso que a Triangulação, mas apresentou predições bastante discrepantes principalmente entre valores mais extremos do conjunto de dados.

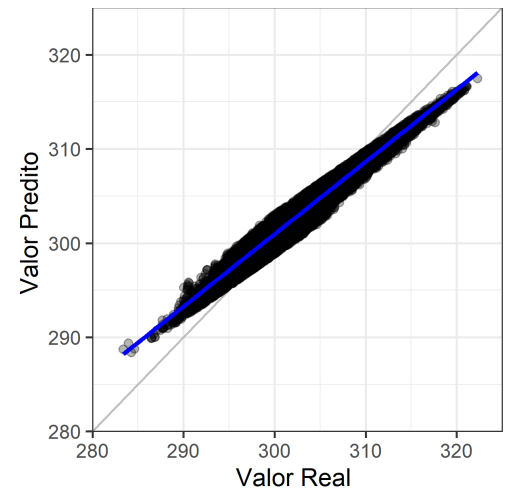

(a) FRK

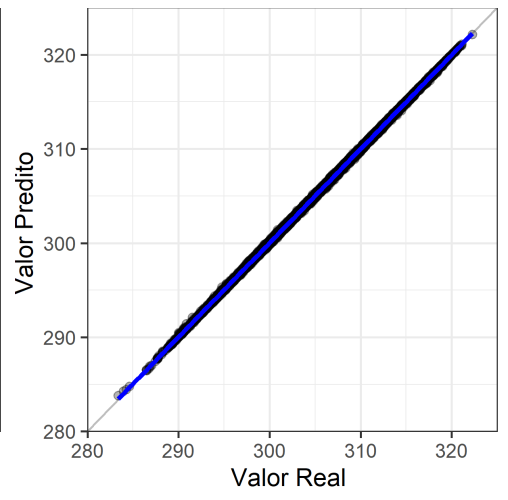

(b) $C A R$

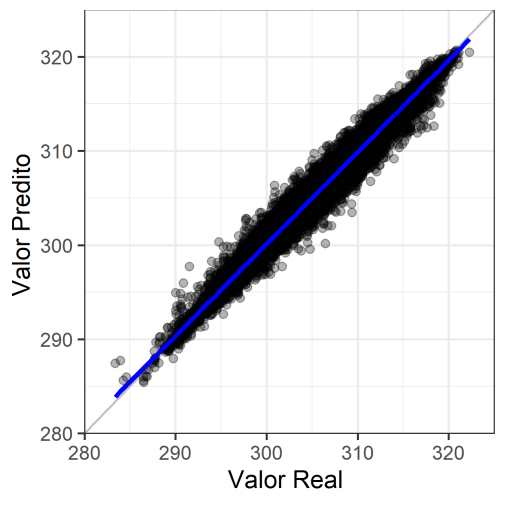

(c) Triangulação

Figura 6.8: Gráficos de dispersão entre o valor real e o valor predito, para os três métodos avaliados

Na Tabela 6.3 apresentamos os erros quadráticos médios das predições para os tempos e localidades com observação disponível e suas raízes. Na Figura 6.9 apresentamos em forma de mapa as raízes dos erros quadráticos médios para os valores preditos ao longo dos tempos em cada localidade. Comparando com a ortofoto apresentada na Figura 6.2, vemos que os erros são menores em áreas que apresentam maior cobertura vegetal, especialmente para o método FRK. Na Figura 6.10 apresentamos em forma de um gráfico de série temporal as raízes dos erros quadráticos médios para os valores preditos nas localidades em cada tempo. A raiz do erro quadrático médio para o método da Triangulação especialmente aparenta ter um comportamento sazonal.

\begin{tabular}{ccc}
\hline \hline & EQM & Raiz do EQM \\
\hline FRK & 2.027 & 1.424 \\
CAR & 0.003 & 0.058 \\
Triangulação & 0.492 & 0.701 \\
\hline
\end{tabular}

Tabela 6.3: Erros quadráticos médios considerando todas as observações.

A Figura 6.11 indica que há um pequeno aumento na raiz do erro quadrático médio quando há mais pontos faltantes numa imagem. 


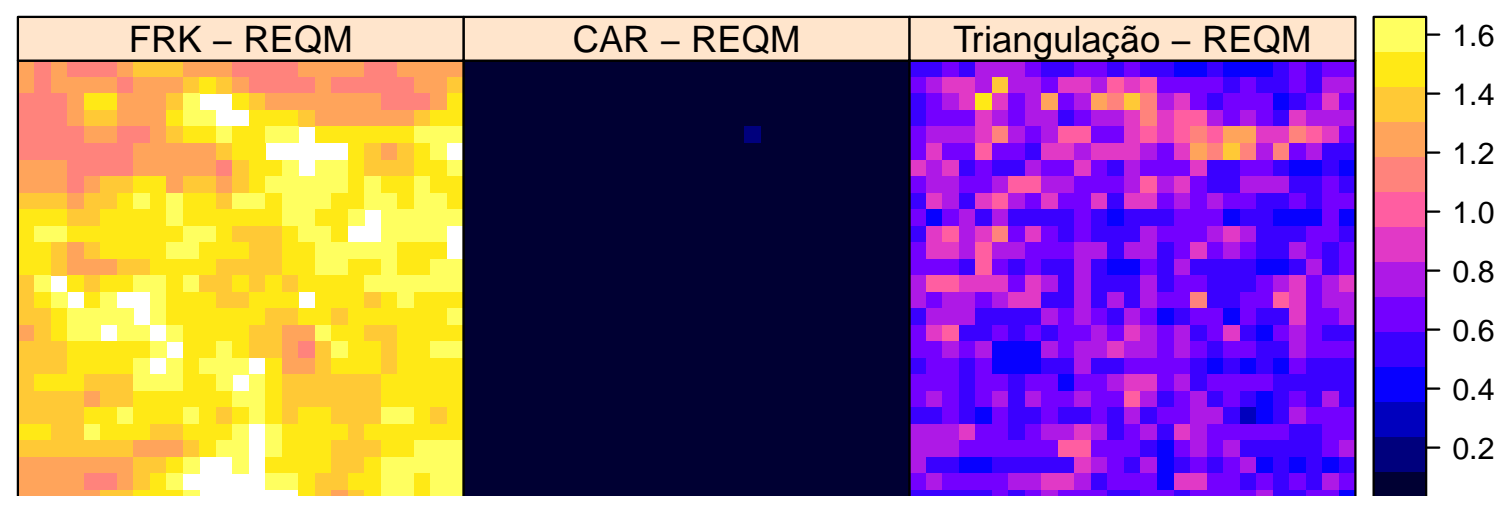

Figura 6.9: As raízes dos erros quadráticos médios em cada método, calculados para cada pixel contido na região e mapeados.

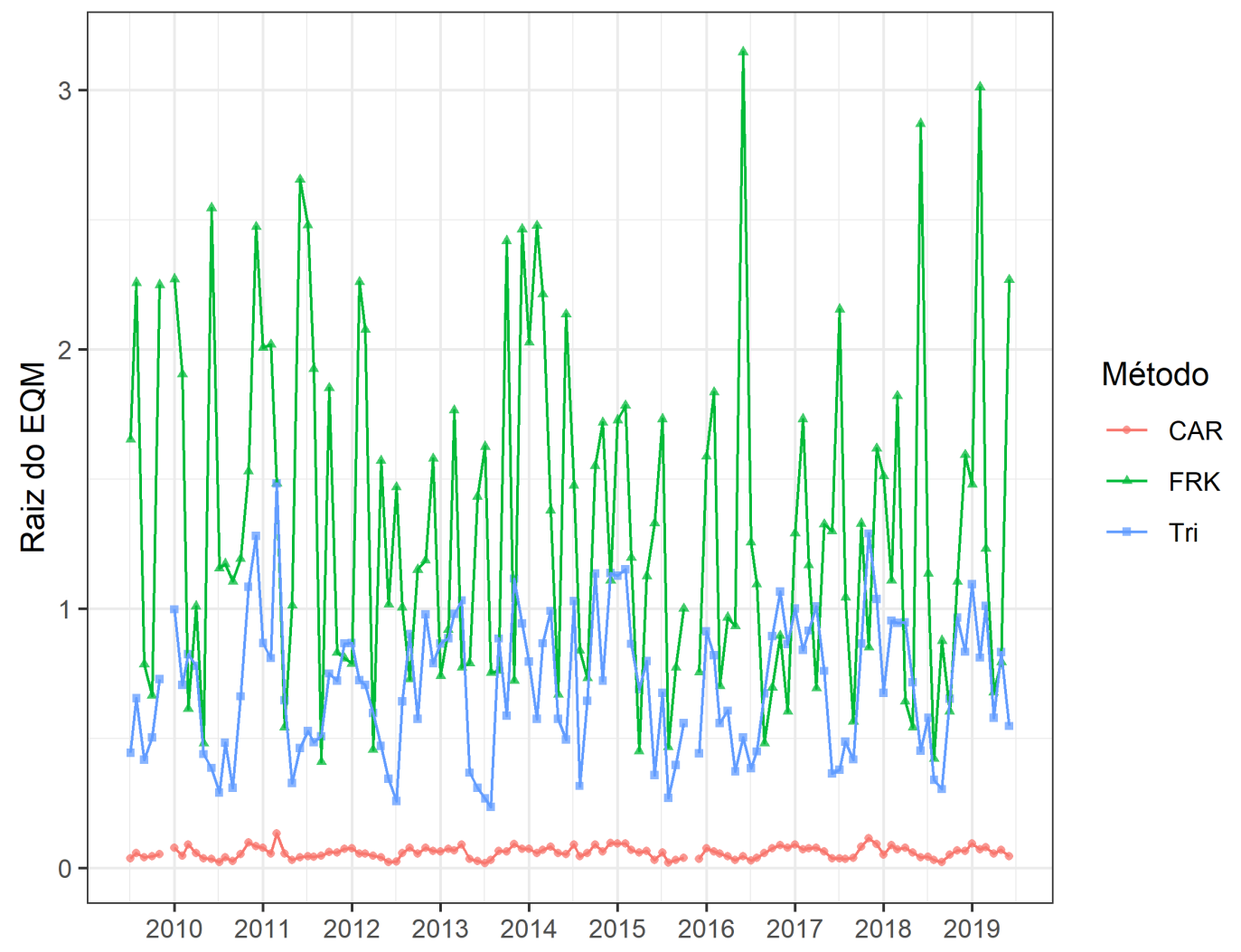

Figura 6.10: Raízes dos erros quadráticos médios por tempo, para cada um dos métodos avaliados. 

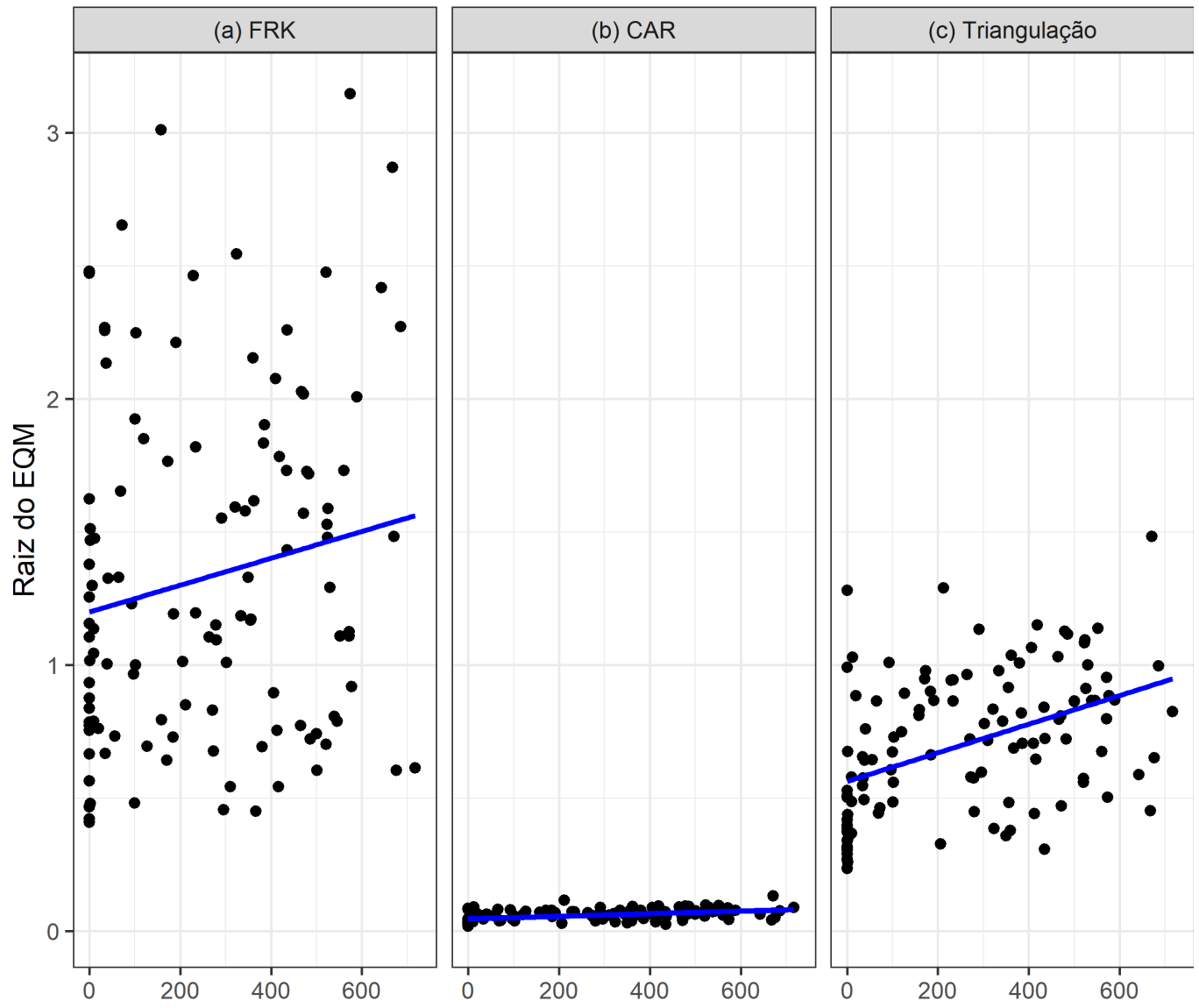

Figura 6.11: Raízes dos erros quadráticos médios pelo número de pontos faltantes numa imagem, para cada um dos métodos avaliados.

Com relação ao tempo de execução, a Tabela 6.4 aponta que, nessa aplicação, o método FRK foi mais rápido, seguido pela triangulação.

\begin{tabular}{cccc}
\hline \hline Krigagem & FRK & CAR & Triangulação \\
\hline 114 & 67 & 399 & 80
\end{tabular}

Tabela 6.4: Tempo, em minutos, para a modelagem e a predição para a aplicação. 



\section{Capítulo 7}

\section{Conclusões e Estudos Futuros}

Neste trabalho, discutimos a questão dos dados faltantes em conjuntos espaçotemporais de imagens oriundas de sistemas de sensoriamento remoto e novas abordagens baseadas em modelos estatísticos que visam interpolar essas observações a fim de se permitir o uso de uma gama de análises que requerem conjuntos de dados completos. No Capítulo 3 apresentamos a Krigagem de Posto Fixo (FRK) e no Capítulo 4 discutimos uma modelagem hierárquica baseada em campos Gaussianos e na função de covariância de Matérn como solução de equações diferenciais parciais estocásticas, utilizando método denominado aproximações de Laplace integradas e aninhadas (INLA).

No Capítulo 5, foi feito estudo de simulação para comparar o método da Krigagem Universal, o método Autorregressivo Condicional (CAR), o método da Krigagem de Posto Fixo (FRK) apresentado no Capítulo 3 e modelos espaço-temporais hierárquicos com triangulações para redução de dimensionalidade apresentados no Capítulo 4. Foram simulados 10 mapas similares aos obtidos através de imagens de satélite com as medições de temperatura de superfície e os métodos foram avaliados através do cálculo do erro quadrático médio para observações mantidas no conjunto e também para observações suprimidas. Uma nova avaliação foi feita para esses conjuntos simulados, acrescidos de um efeito sazonal. O método CAR apresentou os melhores resultados, porém foram os que demandaram maior tempo de execução. Os modelos espaço-temporais hierárquicos apresentaram melhor desempenho que os modelos FRK e demandaram menor tempo de execução.

No Capítulo 6, avaliamos as abordagens selecionadas em imagens obtidas por satélite orbital contendo uma medição remota da temperatura de superfície terrestre em uma área selecionada da Região Metropolitana de São Paulo. Foi verificada a presença de observações faltantes no conjunto de dados, bem como a presença de um efeito sazonal. Os métodos foram avaliados por uma comparação do erro quadrático médio. O método CAR mostrou ter o melhor resultado, mas com um tempo de execução maior. Os modelos FRK mais uma vez apresentaram os maiores erros quadráticos médios, enquanto os modelos que utilizam a triangulação para redução de dimensionalidade apresentaram o EQM maior do que o CAR, mas com tempo de execução muito menor. 
Alguns estudos de interesse para o futuro:

- Avaliar modelos para diferentes distribuições do erro da variável resposta. O pacote FRK foi recentemente expandido para incluir respostas da família exponencial, enquanto o pacote INLA é particularmente robusto quanto às opções de modelos lineares;

- Analisar e aplicar os modelos avaliados em séries de perfis mais variados, como diferentes padrões de não-estacionariedade e anisotropia;

- Avaliar modelos dinâmicos espaço-temporais para levar em consideração com mais detalhamento a possibilidade de mudanças ao longo do tempo que afetam os conjuntos de dados. Na aplicação, por exemplo, alterações no perfil de ocupação do solo vão alterar a temperatura da superfície e a amplitude do efeito sazonal;

- Aplicar os métodos avaliados em conjuntos de diferentes resoluções obtidos de diferentes fontes, a fim de obter um novo conjunto de dados com maiores resoluções espaciais e temporais e avaliar a qualidade dos ajustes obtidos.

- Avaliar os modelos apresentados em conjuntos de imagens multidimensionais, utilizando outros conjuntos como covariáveis no modelo. CHIANN et al. (2018) apresenta uma análise temporal de dados de sensoriamento remoto em que se observa a relação entre remoção de vegetação e aumento em temperatura de superfície;

- Implementar e avaliar diferentes tipos de funções-base para redução de dimensionalidade. A possibilidade do uso de bases mais apropriadas para imagens, como wavelets e curvelets, é bastante promissora na medida em que as imagens se tornam cada vez mais detalhadas. Bases ortogonais também podem ser especialmente úteis para abordar a questão da identificabilidade dos modelos. 


\section{Apêndice A}

\section{Códigos}

\section{A.1 Simulação}

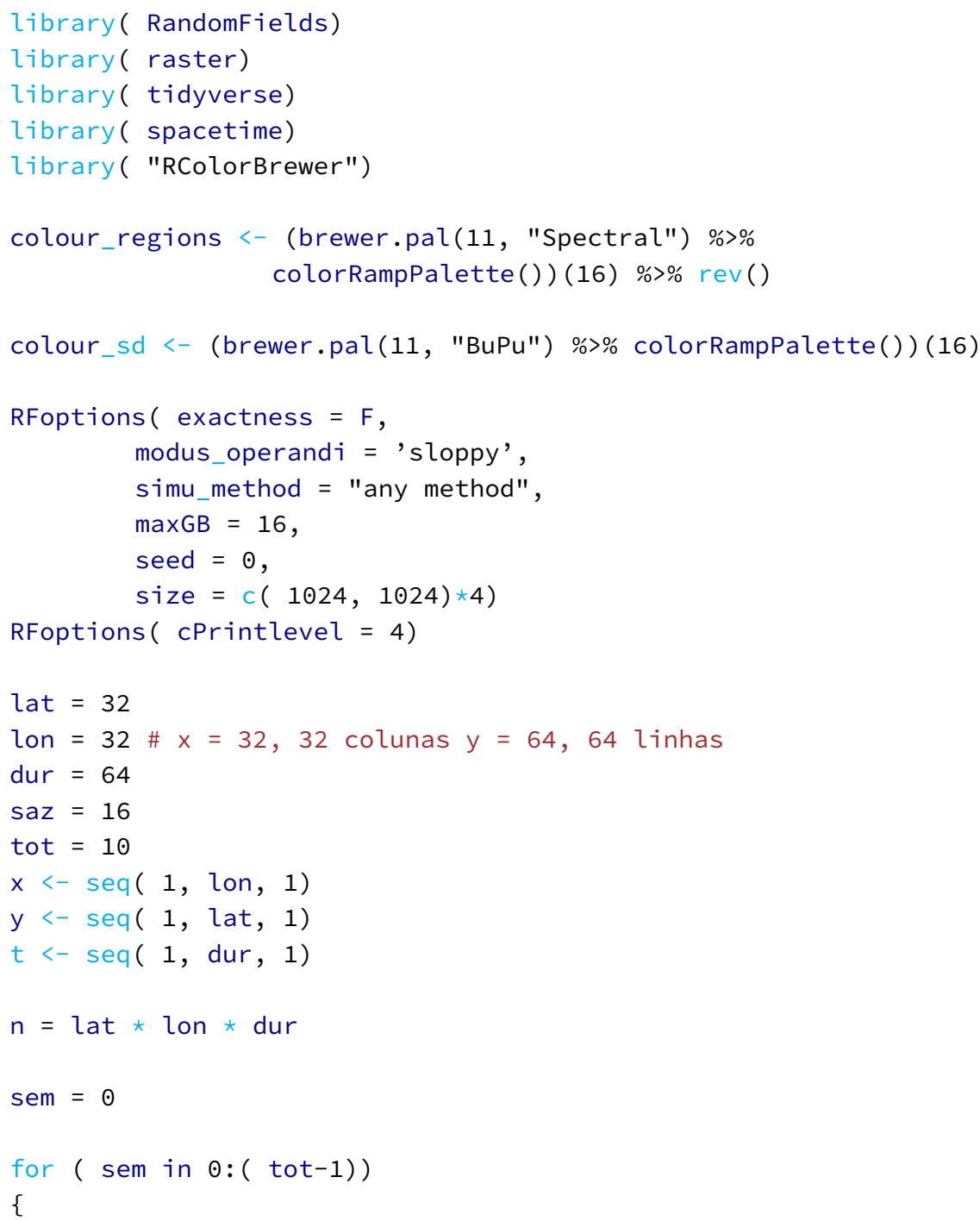




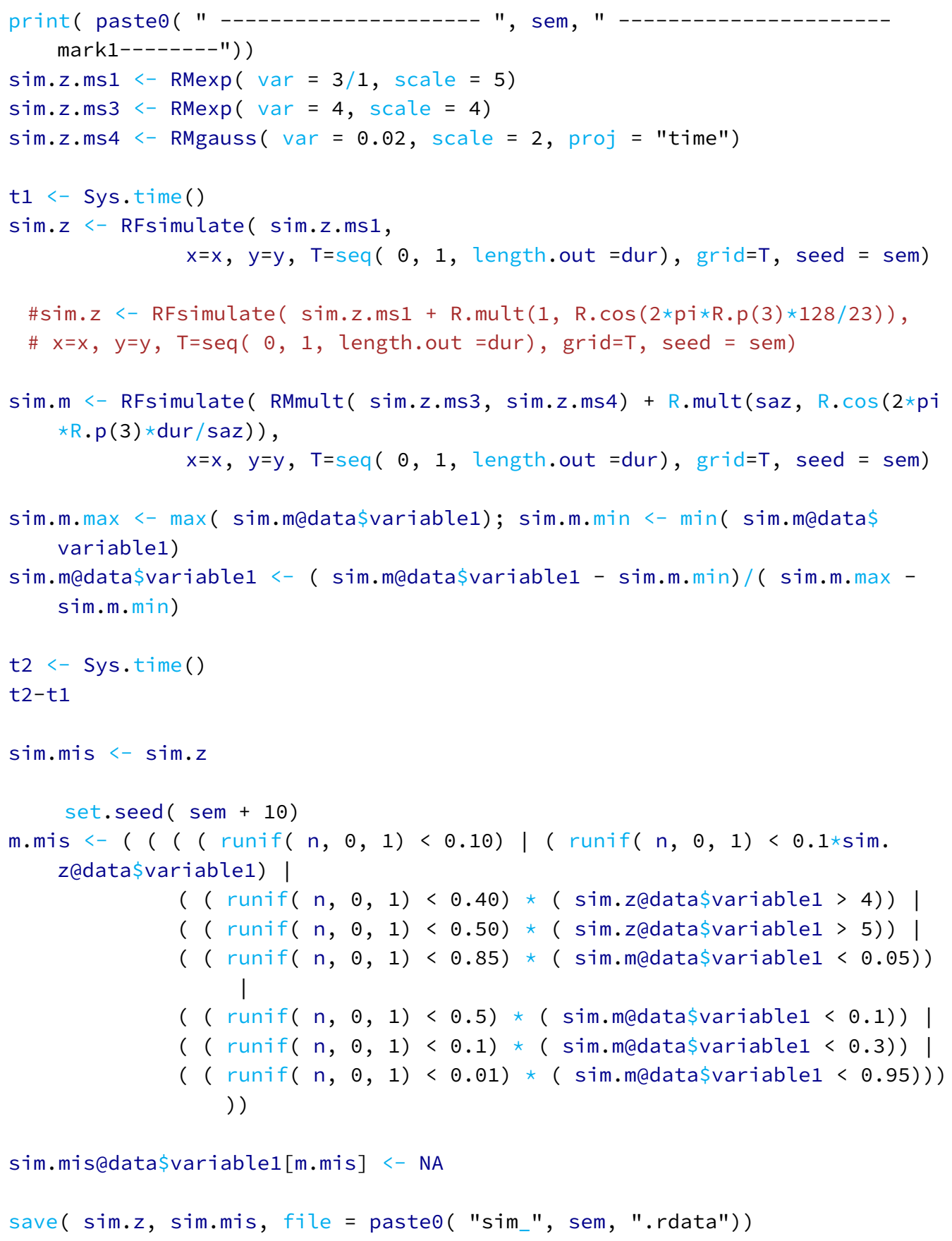

\section{A.2 Krigagem}

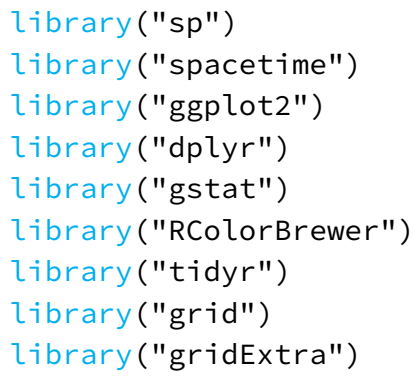


A.2 | KRIGAGEM

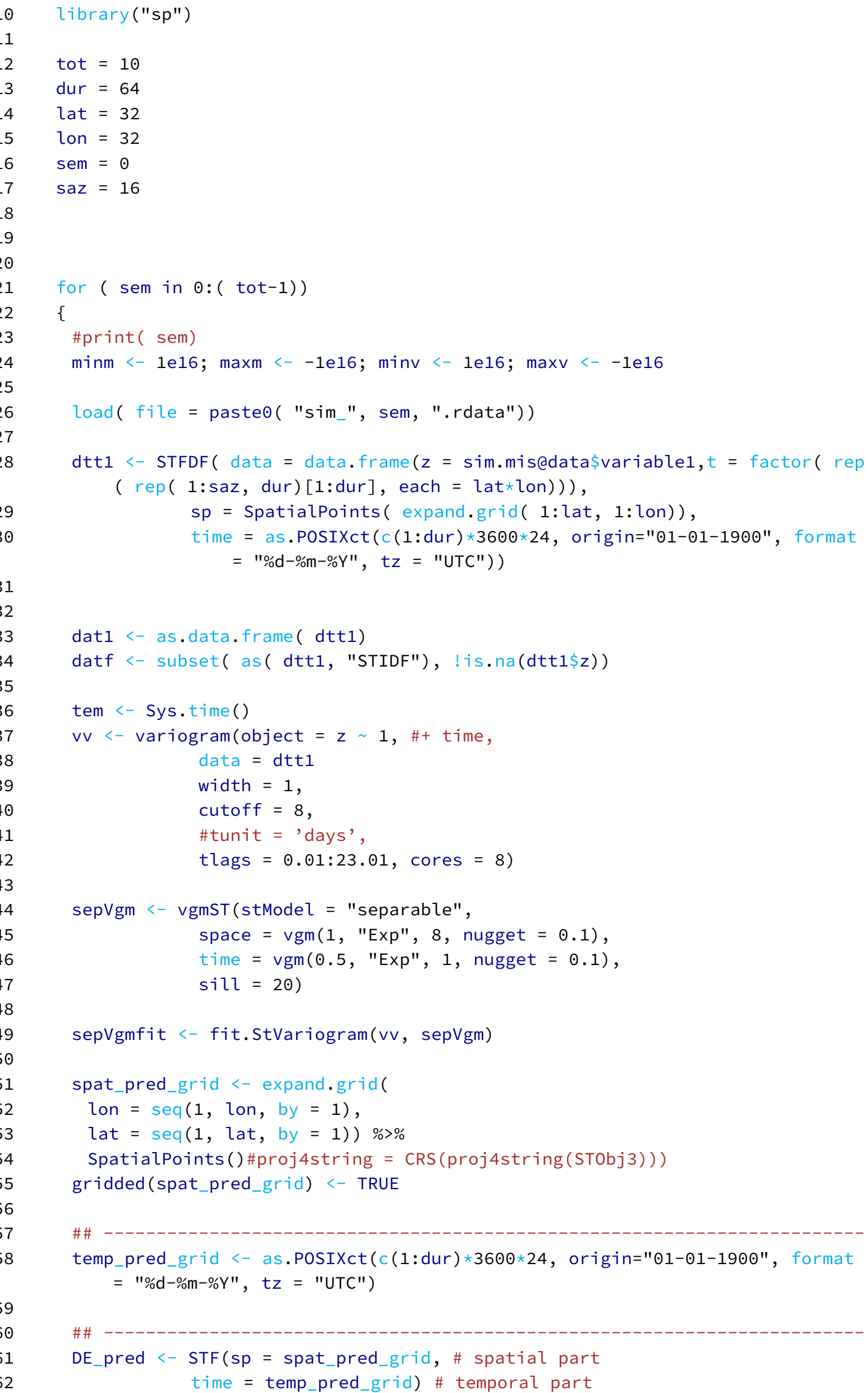




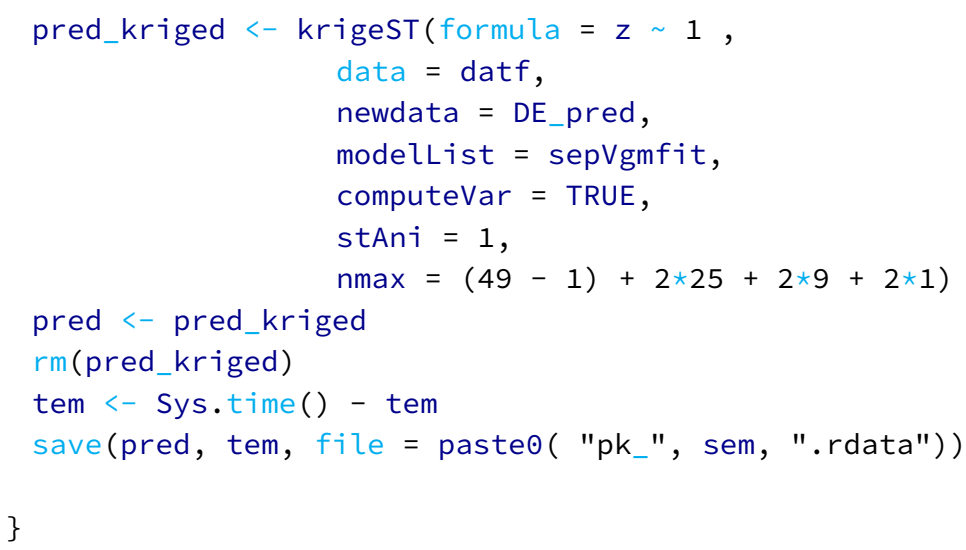

\section{A.3 Krigagem de Posto Fixo (FRK)}

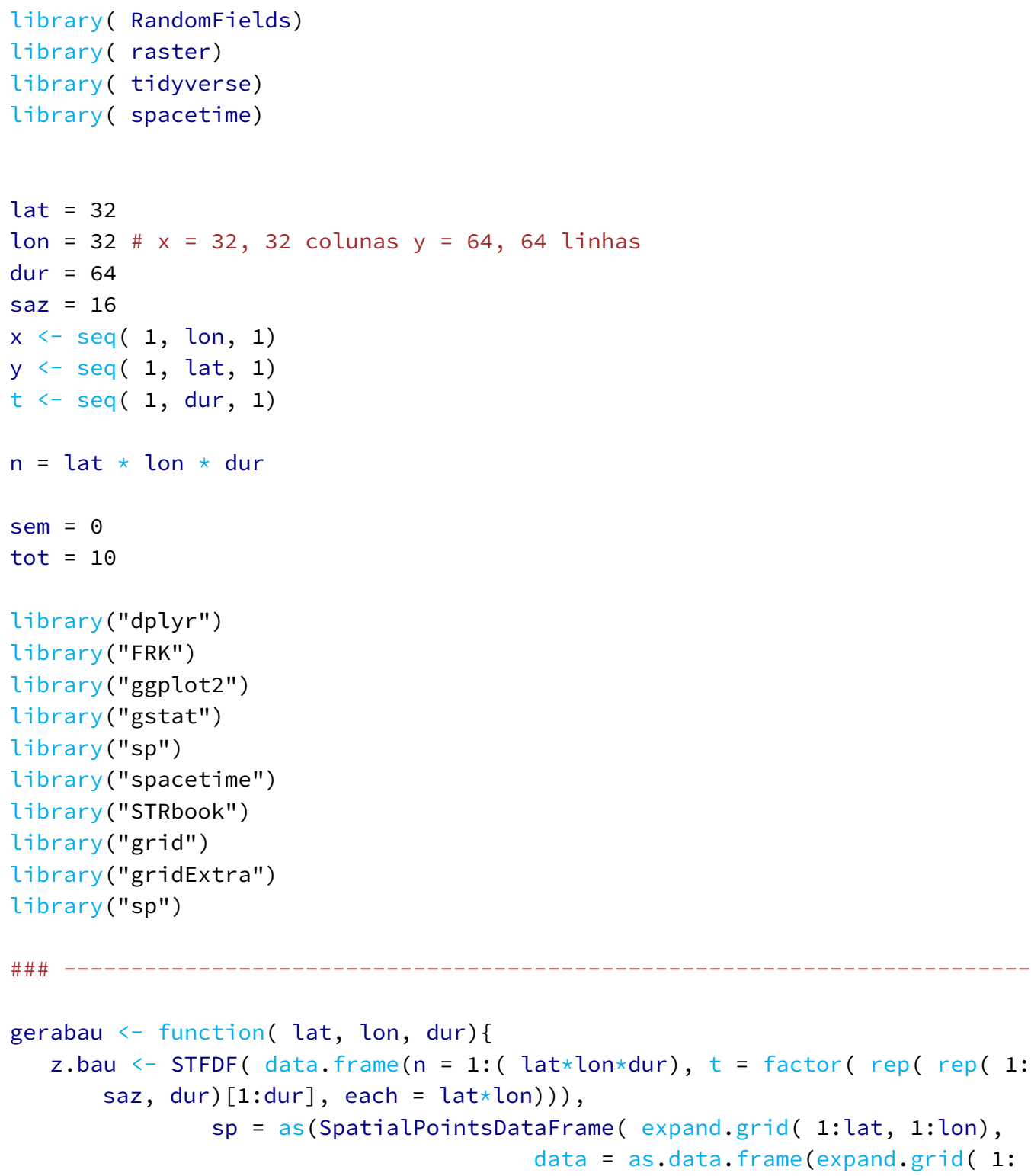


A.3 | KRIGAGEM DE POSTO FIXO (FRK)

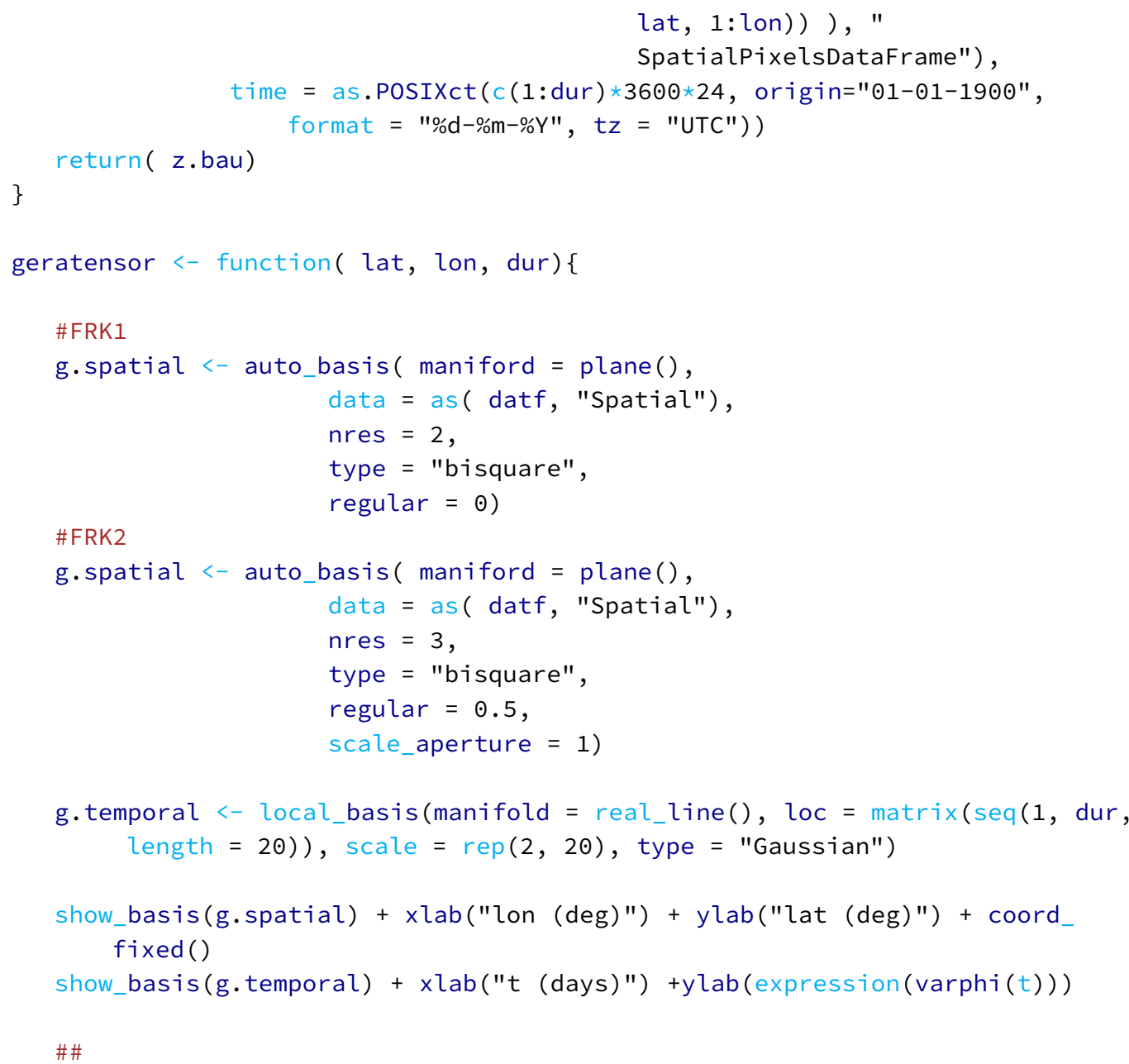


84

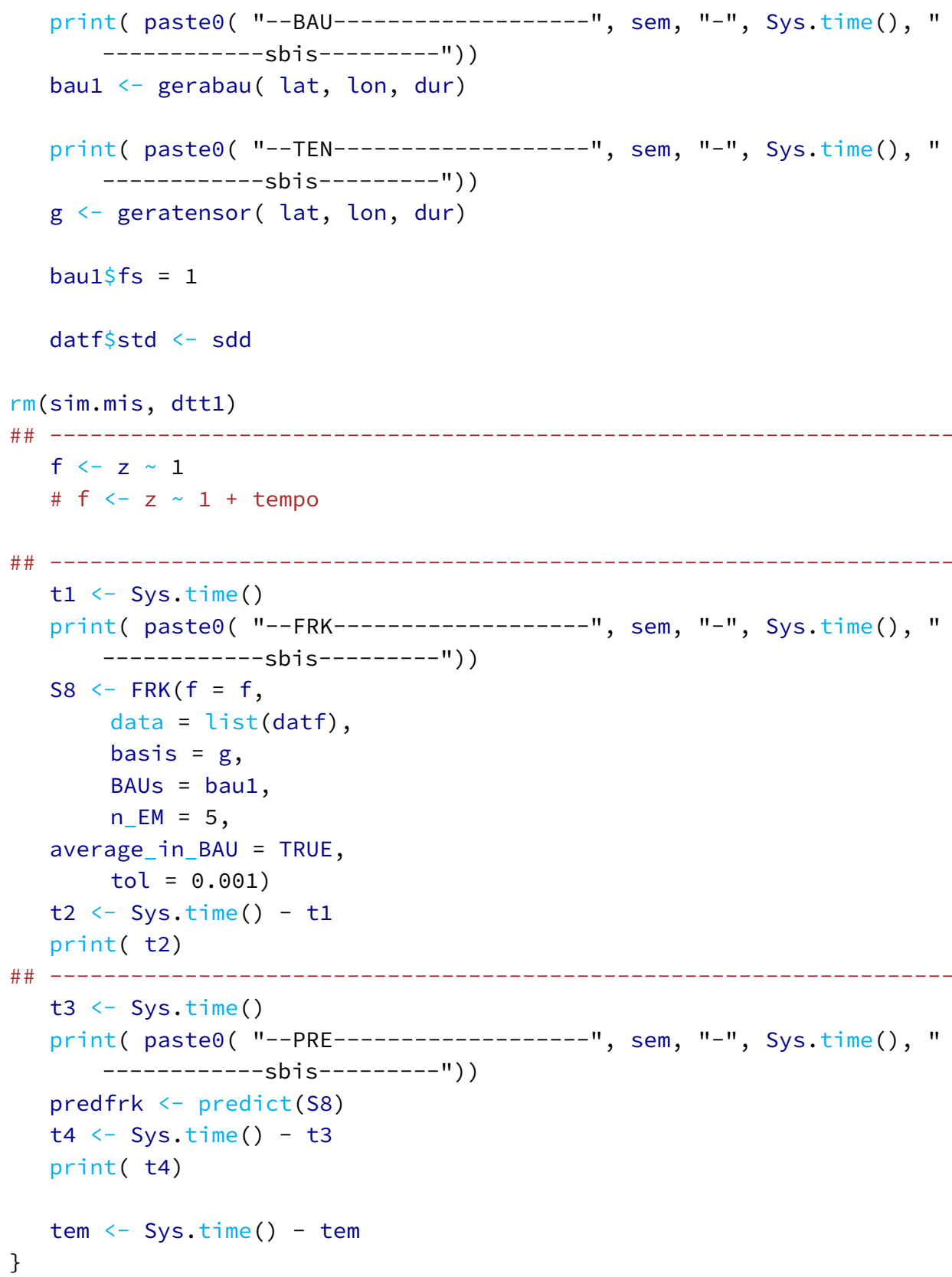

\section{A.4 INLA - CAR}

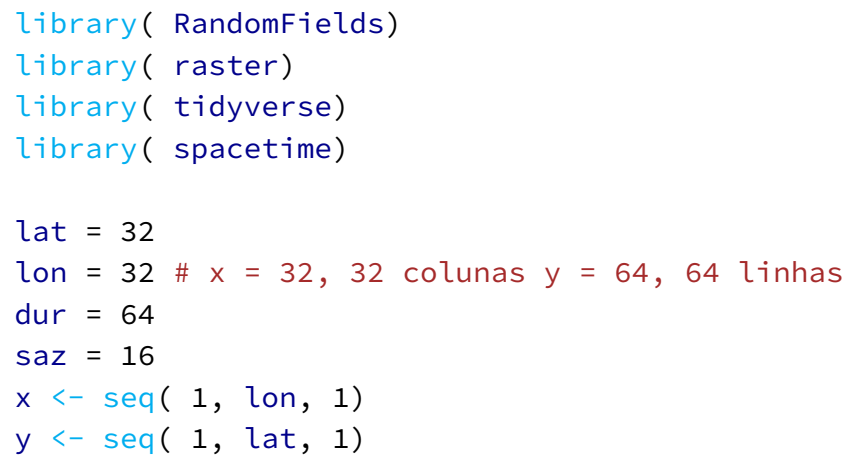




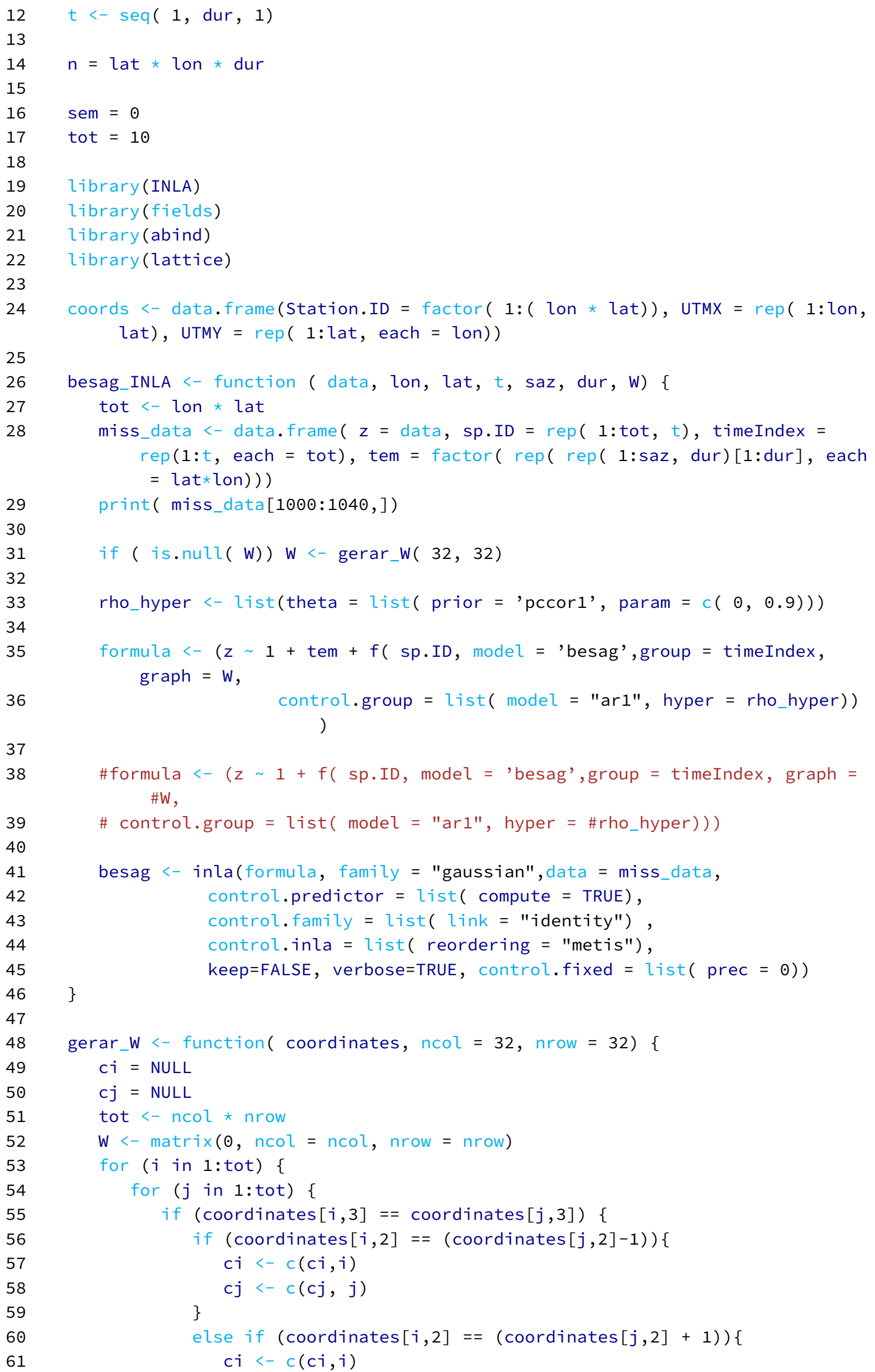




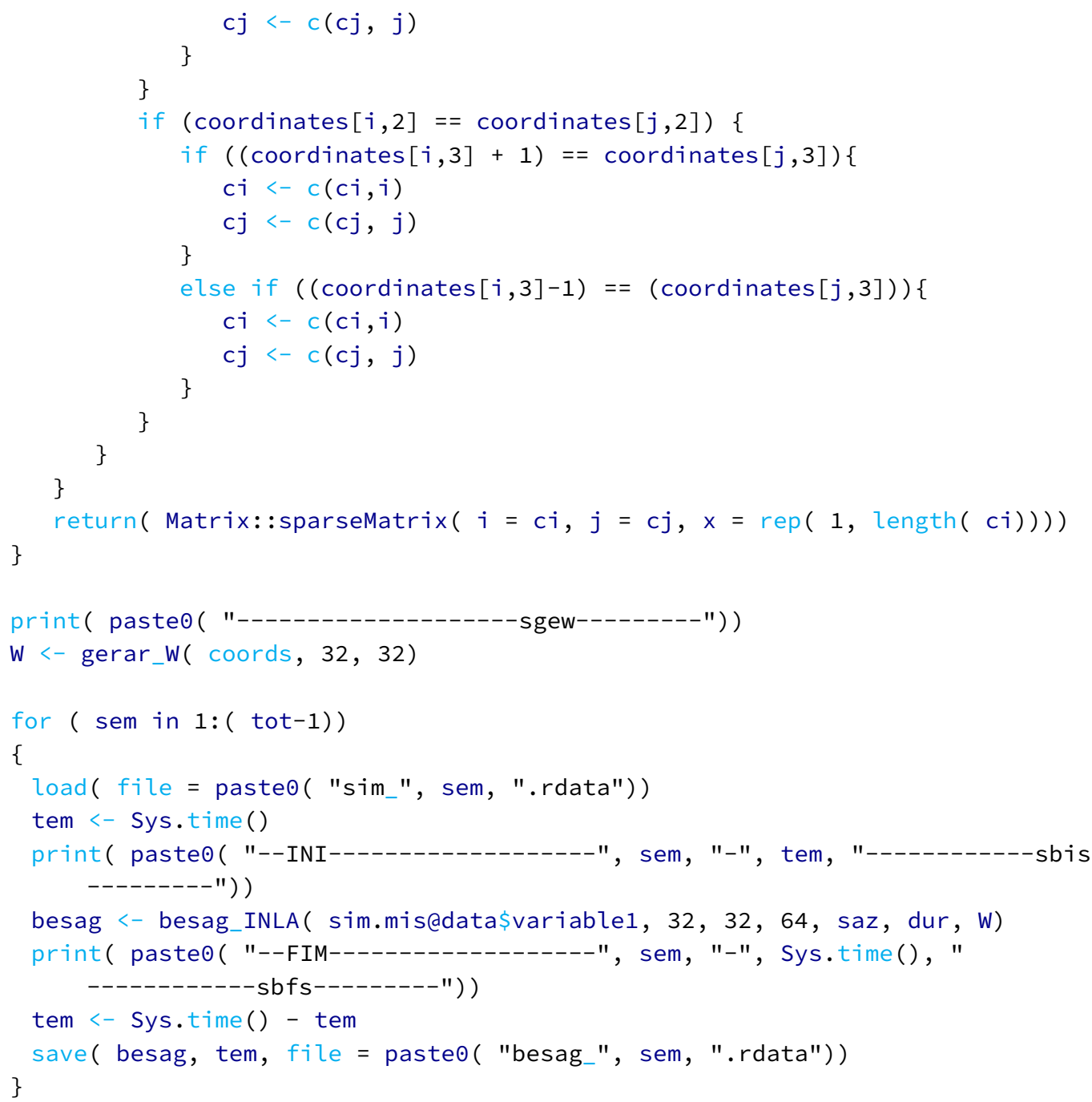

\section{A.5 INLA - Triangulação}

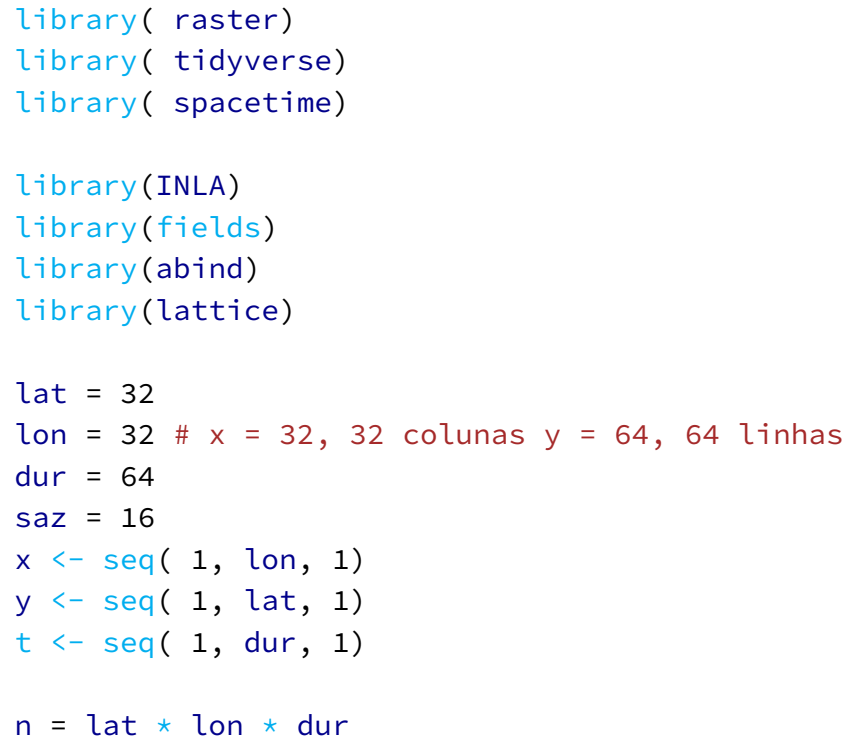




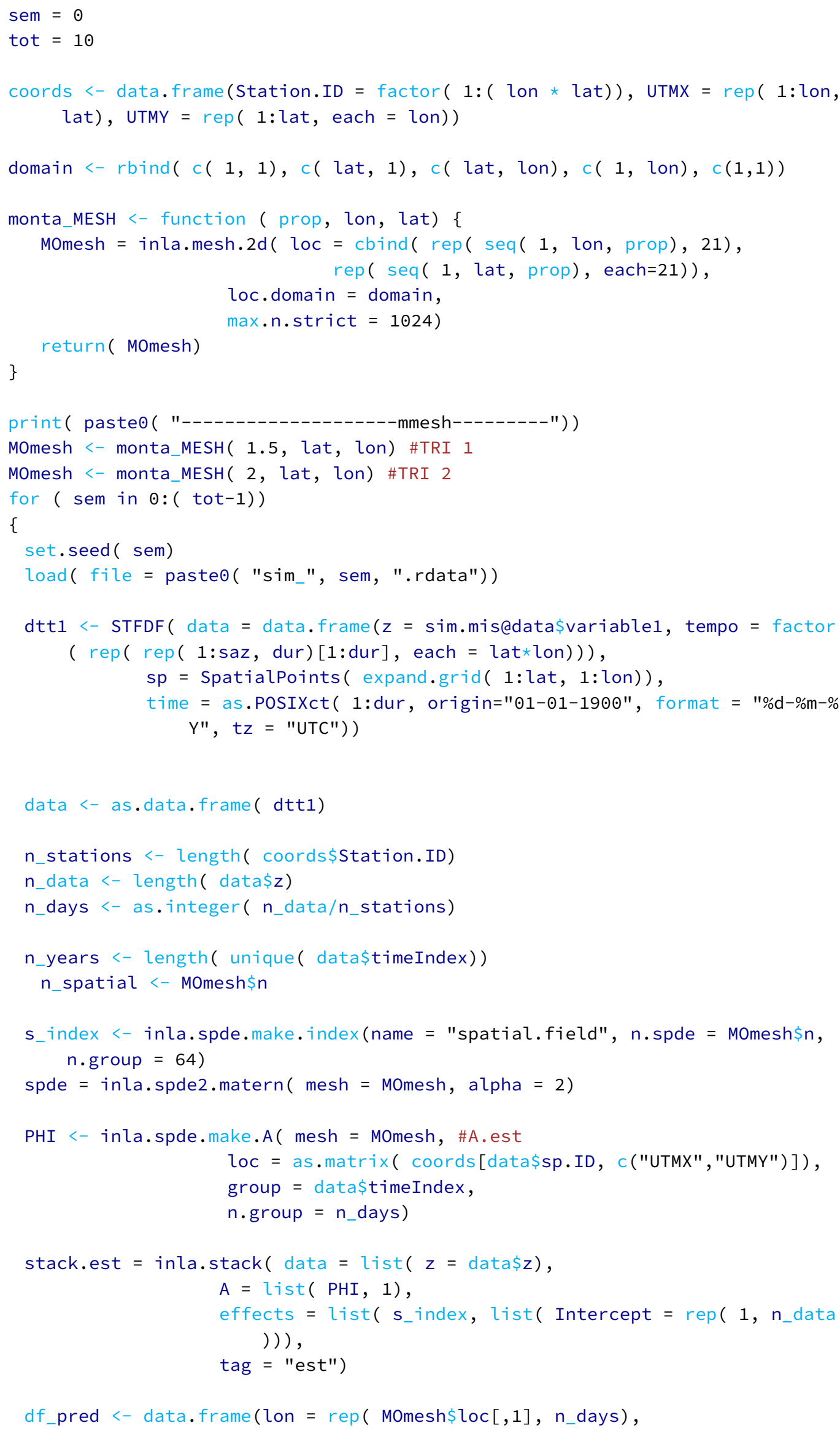




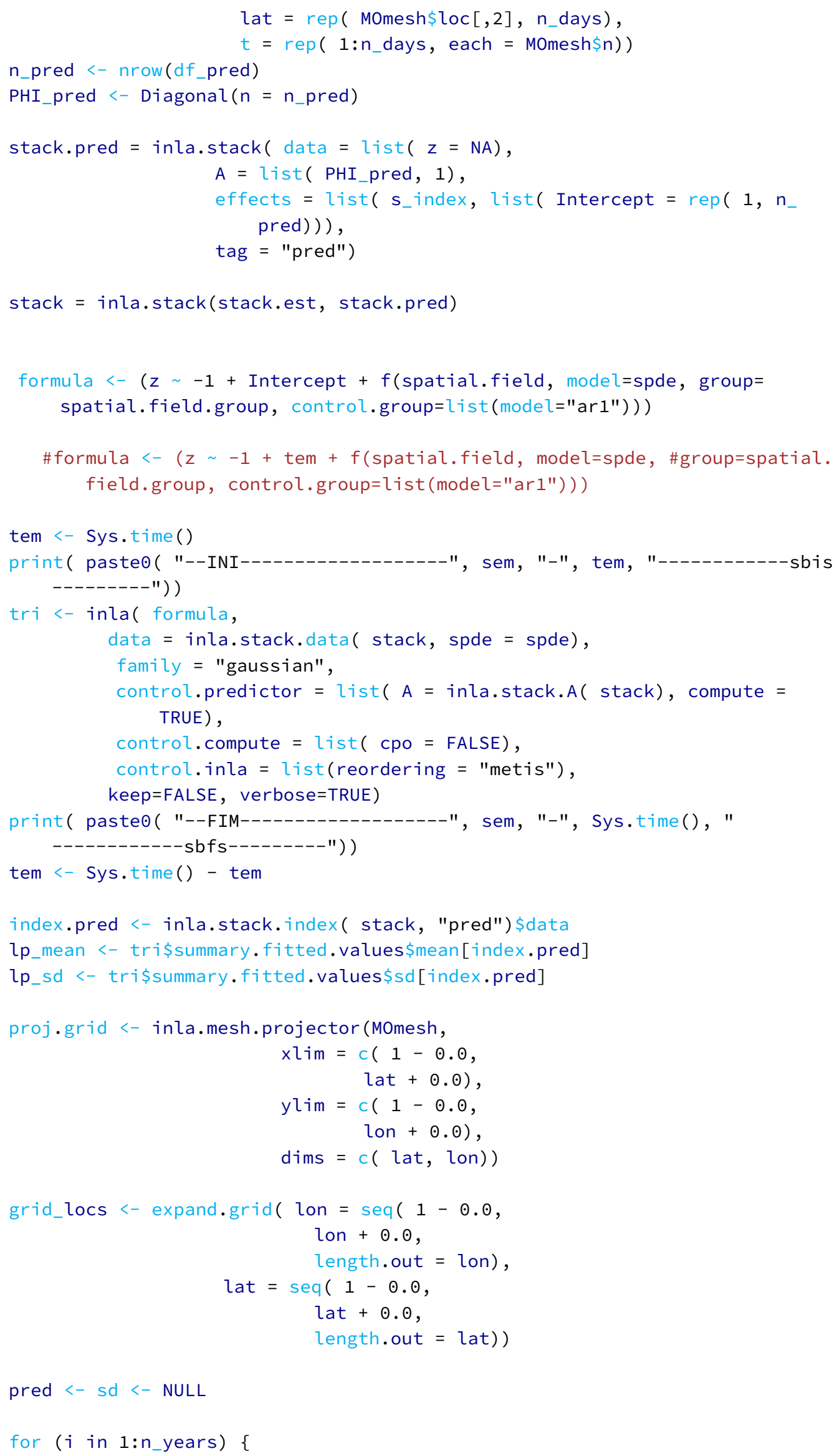




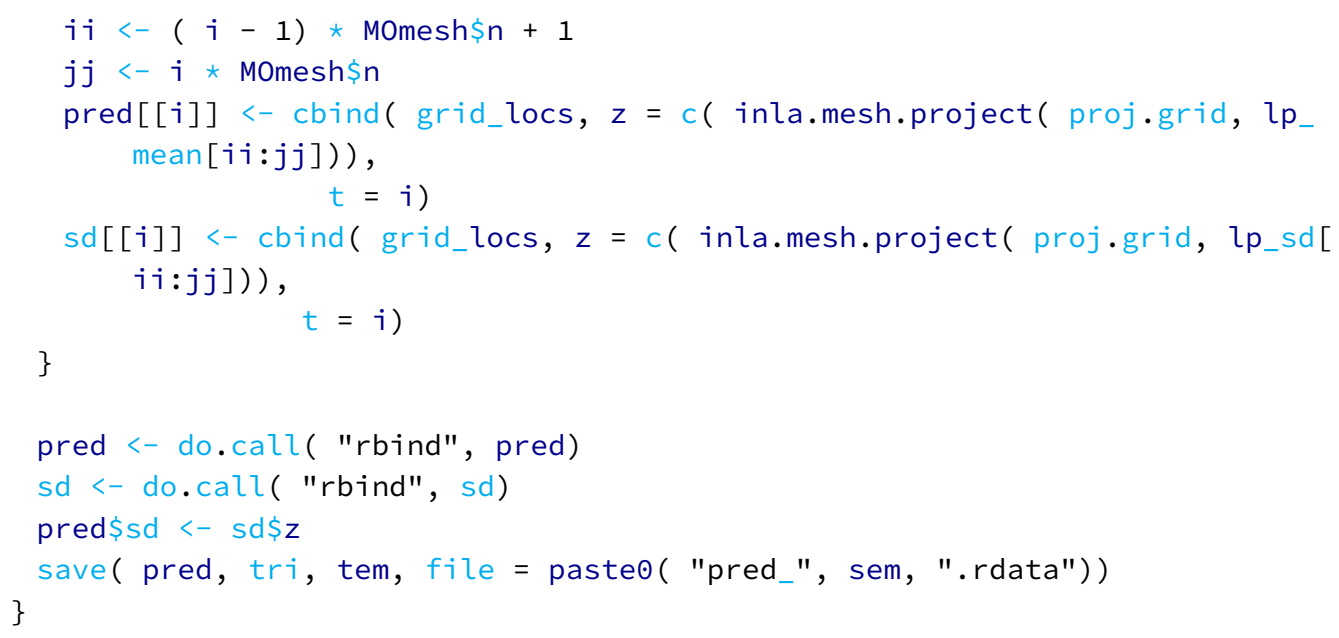

\section{Referências para os pacotes utilizados}

[Auguie 2017] Baptiste AuguiE. gridExtra: Miscellaneous Functions for "Grid"Graphics. $\mathrm{R}$ package version 2.3. 2017. uRL: https://CRAN.R-project.org/package=gridExtra.

[Bivand et al. 2013] Roger S. Bivand, Edzer J. Pebesma e Virgilio Gomez-Rubio. Applied spatial data analysis with R, Second edition. Springer, NY, 2013. URL: http: //www.asdar-book.org/.

[Hijmans 2020] Robert J. Hijmans. raster: Geographic Data Analysis and Modeling. R package version 3.1-5. 2020. URL: https://CRAN.R-project.org/package=raster.

[Lindgren e Håvard Rue 2015] Finn Lindgren e Håvard Rue. "Bayesian spatial modelling with R-INLA”. Em: Journal of Statistical Software 63.19 (2015), pgs. 1-25. URL: http://www.jstatsoft.org/v63/i19/.

[Martins et al. 2013] Thiago G. Martins, Daniel Simpson, Finn Lindgren e Håvard RuE. "Bayesian computing with INLA: New features." Em: Computational Statistics and Data Analysis 67 (2013), pgs. 68-83.

[Neuwirth 2014] Erich Neuwirth. RColorBrewer: ColorBrewer Palettes. R package version 1.1-2. 2014. URL: https://CRAN.R-project.org/package=RColorBrewer.

[Nychka, Furrer et al. 2017] Douglas Nychka, Reinhard Furrer, John Paige e Stephan SAIN. fields: Tools for spatial data. R package version 10.3. Boulder, CO, USA: University Corporation for Atmospheric Research, 2017. DOI: 10.5065/D6W957CT. uRL: https://github.com/NCAR/Fields.

[Pebesma 2004] Edzer J. Pebesma. "Multivariable geostatistics in S: the gstat package". Em: Computers Geosciences 30 (2004), pgs. 683-691.

[Pebesma 2012] Edzer J. Pebesma. "spacetime: spatio-temporal data in R”. Em: fournal of Statistical Software 51.7 (2012), pgs. 1-30. URL: http://www.jstatsoft.org/v51/ $\mathrm{i} 07 /$. 
[Pebesma e Bivand 2005] Edzer J. Pebesma e Roger S. Bivand. "Classes and methods for spatial data in R”. Em: R News 5.2 (nov. de 2005), pgs. 9-13. URL: https://CRAN. R-project.org/doc/Rnews/.

[Plate e Heiberger 2016] Tony Plate e Richard Heiberger. abind: Combine Multidimensional Arrays. R package version 1.4-5. 2016. URL: https://CRAN.R-project. org/package=abind.

[R Core Team 2019] R Core Team. R: A Language and Environment for Statistical Computing. R Foundation for Statistical Computing. Vienna, Austria, 2019. uRL: https: //www.R-project.org/.

[SARKAR 2008] Deepayan SARKAR. Lattice: Multivariate Data Visualization with R. ISBN 978-0-387-75968-5. New York: Springer, 2008. uRL: http://lmdvr.r-forge.r-project. org.

[Schlather, Malinowski, Oesting et al. 2020] Martin Schlather, Alexander MaliNOWski, Marco Oesting et al. RandomFields: Simulation and Analysis of Random Fields. R package version 3.3.8. 2020. URL: https://cran. $r$ - project.org/package= RandomFields.

[Wiскнам 2016] Hadley Wiскнам. ggplot2: Elegant Graphics for Data Analysis. Springer-Verlag New York, 2016. ISBN: 978-3-319-24277-4. URL: https://ggplot2. tidyverse.org.

[Wickham, Averick et al. 2019] Hadley Wickham, Mara Averick et al. "Welcome to the tidyverse”. Em: Journal of Open Source Software 4.43 (2019), pg. 1686. Dor: 10.21105/joss.01686.

[Wickham, François et al. 2020] Hadley Wickham, Romain François, Lionel Henry e Kirill Müller. dplyr: A Grammar of Data Manipulation. R package version 1.0.0. 2020. URL: https://CRAN.R-project.org/package $=$ dplyr.

[Wickham e Henry 2020] Hadley Wickham e Lionel Henry. tidyr: Tidy Messy Data. R package version 1.1.0. 2020. URL: https://CRAN.R-project.org/package=tidyr.

[Zammit-Mangion 2020] Andrew Zammit-Mangion. FRK: Fixed Rank Kriging. R package version 0.2.2.1. 2020. URL: https://CRAN.R-project.org/package=FRK. 


\section{Referências}

[BERKE 1998] Olaf BERKE. "On spatiotemporal prediction for on-line monitoring data". Em: Communication in Statistics- Theory and Methods 27 (9 1998), pgs. 2343-2369 (citado na pg. 2).

[Besag 1974] Julian Besag. "Spatial interaction and the statistical analysis of lattice systems”. Em: Fournal of the Royal Statistical Society Series B (Methodological) 36 (2 1974), pgs. 192-236 (citado nas pgs. 14, 27).

[BesAg 1981] Julian Besag. "On a system of two-dimensional recurrence equations". Em: Journal of the Royal Statistical Society. Series B (Methodological) 43.3 (1981), pgs. 302-309 (citado nas pgs. 28, 34).

[Blangiardo e Cameletti 2015] Marta Blangiardo e Michela Cameletti. "Spatial and Spatio-temporal Bayesian Models with R-INLA”. Em: John Wiley \& Sons, Ltd, 2015. Cap. 1, pgs. 1-18 (citado nas pgs. 3, 5, 6, 23).

[Blangiardo, CAmeletti et al. 2013] Marta Blangiardo, Michela Cameletti, Gianluca BAIo e Håvard RuE. "Spatial and spatio-temporal models with R-INLA". Em: Spatial and Spatio-temporal Epidemiology 4 (mar. de 2013) (citado na pg. 23).

[Bolin 2015] David Bolin. Gaussian Markov random fields. Notas de aula. Jan. de 2015 (citado nas pgs. 27, 28).

[Box e Jenkins 2015] George E. P. Box e Gwilym M. Jenkins. Time Series Analysis: Forecasting and Control. $5^{\text {a }}$ ed. San Francisco: Holden Day, 2015 (citado na pg. 14).

[CAmeletti et al. 2013] Michela Cameletti, Finn Lindgren, Daniel Simpson e Håvard RuE. "Spatio-temporal modeling of particulate matter concentration through the SPDE approach". Em: AStA Advances in Statistical Analysis 97 (2 2013), pgs. 109131 (citado nas pgs. 23, 25, 28, 49). 
[Chiann et al. 2018] Chang Chiann, Michel Soares e Vinicius S. M. Alves. Relatório de análise estatística sobre o projeto: "O impacto da supressão da vegetação no microclima urbano”. Rel. técn. 2018 (citado na pg. 62).

[Cliff e Ord 1975] Andrew D. Cliff e J. Keith Ord. "Space-time modelling with an application to regional forecasting”. Em: Transactions of the Institute of British Geographers 64 (1975), pgs. 119-128 (citado na pg. 14).

[Cressie 1993] Noel Cressie. Statistics for Spatial Data. 1st. John Wiley \& Sons, 1993 (citado nas pgs. 2, 14).

[Cressie e Huang 1999] Noel Cressie e Hsin-Cheng Huang. "Classes of nonseparable, spatio-temporal stationary covariance functions”. Em: fournal of the American Statistical Association 94.448 (1999), pgs. 1330-1340 (citado na pg. 17).

[Cressie e Johannesson 2008] Noel Cressie e Gardar Johannesson. "Fixed rank kriging for very large spatial data sets". Em: Journal of the Royal Statistical Society: Series B (Statistical Methodology) 70.1 (2008), pgs. 209-226 (citado nas pgs. 2, 3, 15, $18,31)$.

[Cressie, Shi et al. 2010] Noel Cressie, Tao Shi e Emily L. Kang. "Fixed rank filtering for spatio-temporal data”. Em: fournal of Computational and Graphical Statistics 19.3 (2010), pgs. 724-745 (citado nas pgs. 5, 18).

[Cressie e Wikle 2011] Noel Cressie e Christopher K. Wikle. Statistics for SpatioTemporal Data. 1st. bla. John Wiley \& Sons, 2011 (citado nas pgs. 6, 15).

[Ehlers 2009] Ricardo S. Ehlers. Análise de Séries Temporais. Notas de aula. 2009 (citado na pg. 6).

[EMPLASA 2010] EMPRESA PAULISTA DE PLANEJAMENTO METROPOLITANO EMPLASA. Projeto de Atualização Cartográfica do Estado de São Paulo. 1 imagem ortorretificada, color. Levantamento aerofotogramétrico 2010/2011. 2010. URL: http: //datageo.ambiente.sp.gov.br (acesso em 10/07/2021) (citado na pg. 51).

[EMPLASA 2019] EMPRESA PAULISTA DE PLANEJAMENTO METROPOLITANO EMPLASA. Projeto de Atualização Cartográfica do Estado de São Paulo. 2019. URL: http://datageo.ambiente.sp.gov.br (acesso em 10/07/2021) (citado na pg. 51).

[ESDIS 2019] Earth Science Data Information System - ESDIS. Earthdata Search. 2019. URL: https://search.earthdata.nasa.gov/ (acesso em 12/08/2019) (citado na pg. 49). 
REFERÊNCIAS

[Ferreira 2019] Luciana S. Ferreira. "Vegetação, temperatura de superfície e morfologia urbana: um retrato da região metropolitana de São Paulo”. Tese de dout. Faculdade de Arquitetura e Urbanismo, 2019 (citado nas pgs. 1, 2).

[Furrer et al. 2006] Reinhard Furrer, Marc G. Genton e Douglas Nychka. "Covariance tapering for interpolation of large spatial datasets”. Em: Journal of Computational and Graphical Statistics 15.3 (2006), pgs. 502-523 (citado na pg. 2).

[Gräler et al. 2016] Benedikt Gräler, Edzer Pebesma e Gerard Heuvelink. "SpatioTemporal Interpolation using gstat”. Em: The R fournal 8.1 (2016), pgs. 204-218 (citado nas pgs. 2, 34).

[Gribov e KrivoruchKo 2004] Alexander Gribov e Konstantin KrivoruchKo. "Geostatistical mapping with continuous moving neighborhood". Em: Mathematical Geosciences 36 (2 2004), pgs. 267-281 (citado na pg. 2).

[Henderson e Searle 1981] Harold V. Henderson e Shayle R. Searle. "On deriving the inverse of a sum of matrices". Em: SIAM Review 23.1 (1981), pgs. 53-60 (citado na pg. 22).

[Huang e Cressie 1996] Hsin-Cheng Huang e Noel Cressie. "Spatio-temporal prediction of snow water equivalent using the kalman filter". Em: Computational Statistics and Data Analysis 22 (2 1996), pgs. 159-175 (citado na pg. 2).

[IBGE 2002] INSTITUTO BRASILEIRO DE GEOGRAFIA E ESTATÍSTICA - IBGE. Censo Brasileiro de 2000. 2002 (citado na pg. 52).

[JIN 2017] Esther Y. JIN. "Estrutura de vizinhanças espaciais nos modelos autorregressivos e de médias móveis espaço-temporais STARMA”. Diss. de mestr. IME-USP, 2017 (citado nas pgs. 14, 32).

[Kaluzny et al. 1998] Stephen P. Kaluzny, Silvia C. Vega, Tamre P. Cardoso e Alice A. Shelly (AUTH.) S+SpatialStats: User's Manual for Windows ${ }^{\circledR}$ and UNIX®. Springer New York, 1998 (citado na pg. 5).

[Katzfuss e Cressie 2011] Matthias Katzfuss e Noel Cressie. "Spatio-temporal smoothing and em estimation for massive remote-sensing data sets". Em: fournal of Time Series Analysis 32 (4 2011) (citado na pg. 20). 
[Kissling e Carl 2008] Daniel W. Kissling e Gudrun Carl. "Spatial autocorrelation and the selection of simultaneous autoregressive models". Em: Global Ecology and Biogeography 17.1 (2008), pgs. 59-71 (citado na pg. 14).

[Kutner et al. 2005] Michael H. Kutner, Christopher J. Nachtsheim, John Neter e William Li. Applied linear statistical models. 5th ed. The McGraw-Hill/Irwin series operations and decision sciences. McGraw-Hill Irwin, 2005 (citado na pg. 13).

[LAird e Ware 1982] Nan M. Laird e James H. Ware. "Random-effects models for longitudinal data". Em: Biometrics 38 (4 1982), pgs. 963-974 (citado nas pgs. 18, 21).

[Lindgren, Håvard Rue et al. 2011] Finn Lindgren, Håvard Rue e Johan Lindström. "An explicit link between Gaussian fields and Gaussian Markov random fields: the stochastic partial differential equation approach". Em: fournal of the Royal Statistical Society: Series B (Statistical Methodology) 73.4 (2011), pgs. 423-498 (citado nas pgs. $2,23,25-27,34)$.

[Mardia et al. 1998] Kanti V. Mardia, Colin Goodall, Edwin J. Redfern e Francisco J. Alonso. "The kriged kalman filter". Em: TEST 7 (2 1998), pgs. 217-282 (citado na pg. 2).

[Martino e Riebler 2020] Sara Martino e Andrea Riebler. "Integrated Nested Laplace Approximations (INLA)”. Em: Wiley StatsRef: Statistics Reference Online. American Cancer Society, 2020, pgs. 1-19 (citado na pg. 29).

[Matheron 1963] Georges Matheron. "Principles of geostatistics". Em: Economic Geology 58.8 (dez. de 1963), pgs. 1246-1266 (citado na pg. 15).

[Meiring et al. 1998] Wendy Meiring, Paul D. Sampson e Peter Guttorp. "Space-time estimation of grid-cell hourly ozone levels for assessment of a deterministic model". Em: Environmental and Ecological Statistics 5 (3 1998), pgs. 197-222 (citado na pg. 2).

[Militino et al. 2018] Ana F. Militino, Maria Dolores Ugarte e Unai PÉrez-Goya. "An introduction to the spatio-temporal analysis of satellite remote sensing data for geostatisticians”. Em: Handbook of Mathematical Geosciences: Fifty Years of IAMG. Ed. por B.S. Daya Sagar, Qiuming Cheng e Frits Agterberg. Cham: Springer International Publishing, 2018, pgs. 239-253 (citado na pg. 1). 
REFERÊNCIAS

[Montero et al. 2015] José-María Montero, Gema FernándeZ-Avilés e Jorge Mateu. Spatial and Spatio-Temporal Geostatistical Modeling and Kriging. $1^{\mathrm{a}}$ ed. Wiley Series in Probability and Statistics. Wiley, 2015 (citado na pg. 5).

[Morettin e Toloi 2004] Pedro A. Morettin e Clélia M. C. Toloi. Análise de séries temporais. Edgard Blucher, 2004 (citado nas pgs. 5-7).

[Nguyen et al. 2014] Hai Nguyen, Matthias Katzfuss, Noel Cressie e Amy BraverMAN. "Spatio-temporal data fusion for very large remote sensing datasets". Em: Technometrics 56 (2 2014), pgs. 174-185 (citado na pg. 20).

[Nychka, Bandyopadhyay et al. 2015] Douglas Nychka, Soutir Bandyopadhyay, Dorit Hammerling, Finn Lindgren e Stephan Sain. "A multiresolution gaussian process model for the analysis of large spatial datasets". Em: fournal of Computational and Graphical Statistics 24 (2 2015), pgs. 579-599 (citado na pg. 20).

[Pfeifer e Deutsch 1980] Phillip E. Pfeifer e Stuart J. Deutsch. "A three-stage iterative procedure for space-time modeling”. Em: Technometrics 22.1 (1980), pgs. 35-47 (citado na pg. 14).

[Pu e Yoo 2019] Qiang Pu e Eun-Hye Yoo. "Spatio-temporal modeling of pm 2.5 concentrations with missing data problem: a case study in beijing, china”. Em: International fournal of Geographical Information Science (2019), pgs. 1-25 (citado na pg. 49).

[R Core Team 2020] R Core Team. R: A Language and Environment for Statistical Computing. R Foundation for Statistical Computing. Vienna, Austria, 2020 (citado na pg. 32).

[Richards e Jia 2005] John A. Richards e Xiuping JiA. Remote Sensing Digital Image Analysis: An Introduction. 4th. Springer, 2005 (citado na pg. 1).

[RIVOIRARd e Romary 2011] Jacques Rivoirard e Thomas Romary. "Continuity for kriging with moving neighborhood”. Em: Mathematical Geosciences 43 (4 2011), pgs. 469-481 (citado na pg. 2).

[Håvard Rue e Held 2005] Håvard Rue e Leonhard Held. Gaussian Markov random fields: theory and applications. $1^{\mathrm{a}} \mathrm{ed}$. Monographs on statistics and applied probability 104. Chapman \& Hall/CRC, 2005 (citado nas pgs. 2, 10, 23-25). 
[Håvard Rue, Martino et al. 2009] Håvard Rue, Sara Martino e Nicolas Chopin. “Approximate bayesian inference for latent gaussian models by using integrated nested laplace approximations”. Em: fournal of the Royal Statistical Society: Series B (Statistical Methodology) 71 (2 abr. de 2009) (citado nas pgs. 3, 23, 28, 29, 31, 34).

[SAmpson e Guttorp 1992] Paul D. Sampson e Peter Guttorp. "Nonparametric estimation of nonstationary spatial covariance structure". Em: Fournal of the American Statistical Association 87.417 (1992), pgs. 108-119 (citado na pg. 26).

[SASsI 2016] Gilberto P. SAssi. "Estimação de modelos geoestatísticos com dados funcionais usando ondaletas”. Tese de dout. IME-USP, 2016 (citado na pg. 9).

[Schlather, Malinowski, Menck et al. 2015] Martin Schlather, Alexander Malinowski, Peter J. Menck, Marco Oesting e Kirstin Strokorb. "Analysis, simulation and prediction of multivariate random fields with package RandomFields". Em: Journal of Statistical Software 63.8 (2015), pgs. 1-25 (citado na pg. 32).

[SEarle 1982] Shayle R. Searle. Matrix Algebra Useful for Statistics. New York: John Wiley \& Sons, 1982 (citado nas pgs. 16, 19).

[Shumway e Stoffer 2017] Robert H. Shumway e David S. Stoffer. Time Series Analysis and Its Applications. 4th. Springer, 2017 (citado na pg. 6).

[Ver Hoef et al. 2018] Jay M. Ver Hoef, Erin E. Peterson, Mevin B. Hooten, Ephraim M. HANks e Marie-Joseè ForTin. "Spatial autoregressive models for statistical inference from ecological data”. Em: Ecological Monographs 88.1 (2018), pgs. 36-59 (citado na pg. 14).

[WaN 2013] Zhengming WAN. MODIS Land Surface Temperature Products Users' Guide. Dez. de 2013 (citado na pg. 50).

[WaN et al. 2015] Zhengming WaN, Simon Hook e Glynn Hulley. MOD11A2 MODIS/Terra Land Surface Temperature/Emissivity 8-Day L3 Global 1km SIN Grid V006. 2015 (citado nas pgs. 2, 49, 50).

[Whittle 1954] Peter Whittle. "On stationary processes in the plane”. Em: Biometrika 41 (3-4 dez. de 1954) (citado nas pgs. 14, 25).

[Whittle 1963] Peter Whittle. "Stochastic-processes in several dimensions". Em: Bulletin of the International Statistical Institute 40.2 (1963), pgs. 974-994 (citado na pg. 25). 
[Wikle e Cressie 1999] Christopher K. Wikle e Noel Cressie. "A dimension-reduced approach to space-time kalman filtering”. Em: Biometrika 86.4 (1999), pgs. 815-829 (citado na pg. 2).

[Wikle, Zammit-Mangion et al. 2019] Christopher K. Wikle, Andrew ZammitMangion e Noel Cressie. Spatio-Temporal Statistics with $R$. 1st. Último acesso em 15/05/2020. Chapman e Hall/CRC, 2019 (citado nas pgs. 6, 13, 15, 19).

[Zammit-Mangion e Cressie 2021] Andrew Zammit-Mangion e Noel Cressie. FRK: An R Package for Spatial and Spatio-Temporal Prediction with Large Datasets. 2021 (citado nas pgs. 2, 15, 18, 20, 21, 25, 34, 49).

[Zhao e Wall 2004] Yanli Zhao e Melanie M. Wall. "Investigating the use of the variogram for lattice data". Em: fournal of Computational and Graphical Statistics 13 (3 set. de 2004) (citado na pg. 5).

[Zimmermann 2009] Dale L. Zimmermann. Environmetrics - Spatial Statistics. eng. Ed. por Abdel H. El-ShaArawi e Jana Jureckova. UNESCO-EOLSS Publishers, 2009 (citado na pg. 5). 
